\author{
UNIVERSIDADE DE SÃO PAULO \\ INSTITUTO DE FÍSICA DE SÃO CARLOS
}

CRISTIANE YUMI MISE LONDON

\title{
Higher-order perturbative QCD in the Higgs decay to bottom quarks
}

São Carlos 



\section{Higher-order perturbative QCD in the Higgs decay to bottom quarks}

Dissertation presented to the Graduate Program in Physics at the Instituto de Física de São Carlos, Universidade de São Paulo, to obtain the degree of Master of Science.

Concentration area: Theoretical and Experimental Physics

Advisor: Prof. Dr. Diogo Rodrigues Boito

\section{Corrected version}

(Original version available on the Program Unit)

São Carlos 
I AUTHORIZE THE REPRODUCTION AND DISSEMINATION OF TOTAL OR PARTIAL COPIES OF THIS DOCUMENT, BY CONVENTIONAL OR ELECTRONIC MEDIA FOR STUDY OR RESEARCH PURPOSE, SINCE IT IS REFERENCED.

London, Cristiane Yumi Mise

Higher-order perturbative QCD in the Higgs decay to bottom quarks / Cristiane Yumi Mise London; advisor Diogo Rodrigues Boito - corrected version -- São Carlos 2021. $119 \mathrm{p}$.

Dissertation (Master's degree - Graduate Program in Theoretical and Experimental Physics) -- Instituto de Física de São Carlos, Universidade de são Paulo - Brasil, 2021 .

1. Higgs boson. 2. QCD. 3. Padé approximant. 4. Renormalon. I. Boito, Diogo Rodrigues, advisor. II. Title. 
To my family and friends, this would not be possible without you. 



\section{ACKNOWLEDGEMENTS}

First of all, I would like to express my deep appreciation and gratitude to my supervisor Prof. Dr. Diogo Boito, for all the valuable support and extensive knowledge during the last four years, all the way from my undergraduate through this master's degree. His patience and motivation were essential for the development of this project and it is a honor to work and learn under his guidance. I could not have imagined having a better advisor for my master's study.

I also had great pleasure of working with Prof. Dr. Diogo Boito's research group at IFSC-USP, Fábio, Gabriel, Marcus and Josh (even if for only six months), who were always available to help. Thanks should also go to Prof. Dr. Pere Masjuan for his collaboration in this project through the helpful and insightful discussions.

I am deeply indebted to my parents for always being by my side and for all the sacrifices that made this possible. Their encouragement and unconditional love have been invaluable throughout these years. I would like to extend my sincere thanks to my sister for her support, help and for always being my friend.

I would like to thank all my friends, who made these years more pleasant and light with conversations about the most diverse and crazy subjects. Special thanks to Angélica, Flávia, Gabs, Guincho, Letícia, Nícolas and Pedro for all the precious times we shared. The last six years would not have been the same without their advice and friendship. Finally, but not less important, I very much appreciate the continuous support and patience of my boyfriend Lucas, who helped me in whatever way he could every time I needed.

This study was supported financially and institutionally in part by the Coordenação de Aperfeiçoamento de Pessoal de Nível Superior - Brasil (CAPES) Finance Code 001, and the São Paulo Research Foundation (FAPESP), grant \#2018/21050-0. 

"[...] what we know is really very, very little compared to what we still have to know." 



\begin{abstract}
LONDON, C. Y. M. Higher-order perturbative QCD in the Higgs decay to bottom quarks. 2021. 121p. Dissertation (Master in Science) - Instituto de Física de São Carlos, Universidade de São Paulo, São Carlos, 2021.

In the absence of direct observation of physics beyond the Standard Model at the LHC, precise tests both in theory and experiment require higher precision. The perturbative QCD corrections to the decay width of the Higgs boson into bottom quarks is known up to fourth order in the strong coupling, $\alpha_{s}$. This series is divergent and assumed to be asymptotic and its properties are better analyzed if we consider its Borel transform. In this work, we employ the method of Padé approximants to the Borel transform to reconstruct the series of the imaginary part of the scalar quarkantiquark correlator, directly related to the decay width, and study its renormalons and higher-order corrections. We begin by testing this method in the large- $\beta_{0}$ limit of QCD, where the perturbative series is known to all orders, using it as a testing ground to determine the best strategy to build the series at higher orders using only the first four coefficients. In this limit, we observe that varying the renormalization scheme of the strong coupling $\alpha_{s}$ and also employing the D-log Padé approximants can improve the convergence to the original function. We apply this procedure in full QCD in order to predict some of the main features of the series at (yet unknown) higher orders. We estimate the coefficient of order $\alpha_{s}^{5}$ (six loops) in a model-independent way as $-8200 \pm 308$ and we could conclude that, although very small, this correction is important to stabilize the series. Thus, at present, the limiting factors in the precision of the decay rate of Higgs into $b \bar{b}$ are the uncertainties of the strong coupling $\alpha_{s}$ and the bottom-quark mass $m_{b}$.
\end{abstract}

Keywords: Higgs boson. QCD. Padé approximant. Renormalon. 



\section{RESUMO}

LONDON, C. Y. M. QCD perturbativa em ordens altas no decaimento do Higgs em quarks bottom. 2021. 121p. Dissertação (Mestrado em Ciências) Instituto de Física de São Carlos, Universidade de São Paulo, São Carlos, 2021.

Na ausência de observação direta de física além do Modelo Padrão no LHC, testes precisos tanto da teoria quanto experimentais requerem maior precisão. As correções perturbativas em QCD do decaimento do bóson de Higgs em quarks bottom é conhecido até quarta ordem no acoplamento forte, $\alpha_{s}$. Essa série é divergente e assumida assintótica e suas propriedades são melhor analisadas se considerarmos sua transformada de Borel. Nesse trabalho, empregamos o método dos aproximantes de Padé na transformada de Borel para reconstruir a série da parte imaginária do correlator escalar quark-antiquark, que está diretamente relacionado à taxa de decaimento, e estudar seus renôrmalons e suas correções de ordens mais altas. Iniciamos testando esse método no limite large- $\beta_{0}$ da QCD, onde a série perturbativa é conhecida em todas as ordens, usando-o como um laboratório para determinar a melhor estratégia para reconstruir a série em ordens mais altas usando somente os quatro primeiros coeficientes. Nesse limite, observamos que variar o esquema de renormalização do acoplamento forte $\alpha_{s}$ e aplicar os chamados D-log Padés podem melhorar a convergência à função original. Aplicamos o procedimento na QCD completa a fim de prever algumas das características principais da série em ordens superiores (ainda desconhecidas). Estimamos o coeficiente de ordem $\alpha_{s}^{5}$ (seis loops) independente de modelo como $-8200 \pm 308$ e pudemos concluir que, apesar de ser muito pequena, essa correção é importante para estabilizar a série. Portanto, atualmente os fatores limitantes na precisão da taxa de decaimento do Higgs em $b \bar{b}$ são as incertezas do acoplamento forte $\alpha_{s}$ e da massa do quark bottom $m_{b}$.

Palavras-chave: bóson de Higgs. QCD. Aproximante de Padé. Renôrmalon. 



\section{LIST OF FIGURES}

Figure 1 - The potential $V(\phi)$ for a complex scalar field for (a) $\mu^{2}>0$ and (b) $\mu^{2}<0 \ldots \ldots \ldots \ldots \ldots \ldots \ldots \ldots$

Figure 2 - 3-Higgs vertex. . . . . . . . . . . . . . . . . 29

Figure 3 - 4-Higgs vertex. . . . . . . . . . . . . . . . . . 29

Figure 4 - Interaction vertex between Higgs and fermions. . . . . . . . . . . . 29

Figure 5 - Interaction vertex between gluons and quarks. . . . . . . . . . . . . 31

Figure 6 - 3-gluon vertex. . . . . . . . . . . . . . 31

Figure 7 - 4-gluon vertex. . . . . . . . . . . . . . . 31

Figure 8 - One-loop correction to the quark propagator. . . . . . . . . . . 33

Figure 9 - Experimental determination of $\alpha_{s}$ collected by Particle Data Group. 38

Figure 10 - Branching ratios of different decay modes of the SM Higgs as a function of its mass including the uncertainties in 2005 of the quark masses and the QCD coupling. . . . . . . . . . . . . . 42

Figure 11 - One-loop diagram of bottom quarks. . . . . . . . . . . . . . . . . 44

Figure 12 - One-loop massless propagator diagram, where $n_{i}$ is the power of the propagator. . . . . . . . . . . . . . . 4 46

Figure 13 - Two-loop massless propagator diagram, where $n_{i}$ is the power of the propagator. . . . . . . . . . . . . . . . . 4 48

Figure 14 - Diagram for the case $n_{5}=0 \ldots \ldots \ldots \ldots$. . . . . . . . 48

Figure 15 - Diagram for the case $n_{1}=0 \ldots \ldots \ldots \ldots$. . . . . . . . 48

Figure 16 - Diagrams that contribute to the first-order correction. . . . . . . . . 50

Figure 17 - Diagram for the first contribution. . . . . . . . . . . . . 50

Figure 18 - Diagram for the second contribution. . . . . . . . . . . . . 51

Figure 19 - Corrections to the Adler function. . . . . . . . . . . . . 58

Figure $20-B\left[f_{1}\right](u)$ given in Eq. (4.21) adopting $\alpha=0.15$. . . . . . . . . . . 62

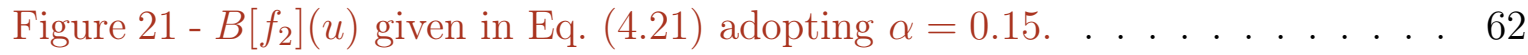

Figure 22 - Comparison between the exact result of $f(z)=\frac{1-z}{(1+4 z)^{2}}$, its Padé Approximant $P_{1}^{1}$ and its Taylor series truncated at second order. . . 64

Figure 23 - Relative error of the first coefficient predicted by $P_{N}^{N}$ obtained from $g(z)=\sqrt{\frac{3+z}{1+z}} \ldots \ldots \ldots \ldots \ldots \ldots \ldots \ldots \ldots \ldots \ldots \ldots \ldots \ldots \ldots \ldots$

Figure 24 - Poles predicted by $P_{13}^{13}$ obtained from $g(z)=\sqrt{\frac{3+z}{1+z}} \ldots \ldots$. . . . . . 67

Figure 25 - Gluon propagator corrected by fermion-loop chains. . . . . . . . . . . 72

Figure 26 - Diagrams that contribute to the first-order correction in the large- $\beta_{0}$ limit. . . . . . . . . . . . . . . . . 73 
Figure 27 - Relative error for the first predicted coefficient $c_{n}$ for each Padé to the imaginary part.

Figure 28 - Borel integral of $F_{L \beta}$ for several Padés to the imaginary part in large- $\beta_{0}$ using $\alpha_{s}\left(m_{H}\right)=0.1125$.

Figure 29 - Relative error for the first predicted coefficient $\widehat{c}_{n}$ for each Padé to the imaginary part using $C=-5 / 3 \ldots \ldots \ldots$. . . . . . .

Figure 30 - Borel integral of $\widehat{F}_{L \beta}^{(C=-5 / 3)}$ for PAs to the imaginary part in large- $\beta_{0}$ using $C=-5 / 3$ and $\tilde{\alpha}_{s}\left(m_{H}\right)=0.1270 \ldots \ldots \ldots$. . . . . .

Figure 31 - Relative error for the first predicted coefficient $c_{n}$ of $F_{L \beta}$ for each PPA after being rebuilt as a function of $m(\mu) \ldots . . . . . .$.

Figure 32 - Borel integral of $\widehat{F}_{L \beta}^{(C=0)}$ for PPAs to the imaginary part in the large- $\beta_{0}$

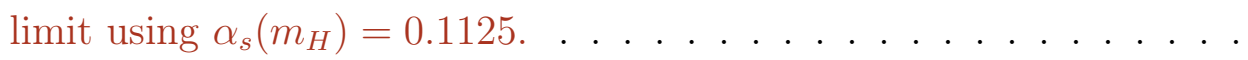

Figure 33 - Relative error for the first predicted coefficient $c_{n}$ of $F_{L \beta}$ in the $\overline{\mathrm{MS}}$ for each PPA constructed in the scheme with $C=-5 / 3$ after being rebuilt as a function of $m(\mu) \ldots \ldots$. . . . . . . . . . 90

Figure 34 - Relative error for the first predicted coefficient $\widehat{r}_{n}$ for each Padé to the second derivative. . . . . . . . . . . . . . . . 91

Figure 35 - Borel integral of $\widehat{D}_{L \beta}^{(C=0)}$ for Padés to the second derivative in large- $\beta_{0}$ with $\alpha_{s}\left(m_{H}\right)=0.1125 \ldots \ldots \ldots \ldots$. . . . . . . . . . . . . . . .

Figure 36 - Relative error for the first predicted coefficient $\widehat{r}_{n}$ of $\widehat{D}_{L \beta}^{(C=0)}$ for each D-log Padé to the second derivative. . . . . . . . . . . . . .

Figure 37 - Borel integral of $\widehat{D}_{L \beta}^{(C=0)}$ for D-log Padés to the second derivative in the large- $\beta_{0}$ limit with $\alpha_{s}\left(m_{H}\right)=0.1125 \ldots \ldots$. . . . . . 93

Figure 38 - Relative error for the first predicted coefficient $\widehat{r}_{n}$ of $\widehat{D}_{L \beta}^{(C=0)}$ in the $\overline{\mathrm{MS}}$ scheme for each D-log Padé to the second derivative constructed in the scheme where $C=-5 / 3 \ldots \ldots$. . . . . . . . . 94

Figure 39 - Dependence on the Higgs decay rate with the renormalization scale $\mu .109$

Figure 40 - Perturbative expansion of the decay rate in QCD at different renormalization scales using the coefficients of Tab. 10 and the integral given in Eq. (6.9). The uncertainty of the Borel integral is due to (a) the strong coupling uncertainty and (b) the different Padé predictions. The uncertainty bars of the points in (b) are due to the errors of the predicted coefficients. . . . . . . . . . . . . . . 110 


\section{LIST OF TABLES}

Table 1 - Coefficients of $F_{L \beta}$ (written in terms of the scale-dependent mass) in the $\overline{\mathrm{MS}}$. The values in blue are the exact ones in the large- $\beta_{0}$ limit. The Padés in white were applied to the Borel transform of $F_{L \beta}$ and the ones in pink to the Borel transform of $\widehat{F}_{L \beta}^{C}$. The terms inside the brackets indicate the scheme where the Padés were constructed. . . . 86

Table 2 - Contribution in percent of the lowest UV and IR poles and the no-pole term to the first 12 coefficients of the $\widehat{F}_{L \beta}^{(C=0)}$ series in the $\overline{\mathrm{MS}}$ scheme. 88

Table 3 - Coefficients $d_{n, 1}$ in the $\overline{\mathrm{MS}}$ predicted by Padés and D-log Padés and the exact value in large- $\beta_{0}$ in blue. The Padés in white were applied to the Borel transform of $\widehat{F}_{L \beta}^{C}$ and the ones in pink to the Borel transform of $\widehat{D}_{L \beta}^{C}$. The PAs with $(C)$ were constructed in the scheme where $C=-5 / 3$, otherwise they were applied in the $\overline{\mathrm{MS}}$ scheme. . . 96

Table 4 - Coefficients $c_{n}$ in the $\overline{\mathrm{MS}}$ scheme predicted by the Padés to the Borel transform of the reduced imaginary part in QCD . . . . . . . . . 100

Table 5 - Coefficients $r_{n}$ in the $\overline{\mathrm{MS}}$ scheme predicted by the Padés to the Borel transform of the reduced second derivative in QCD. . . . . . . . . . 102

Table 6 - Coefficients $r_{n}$ in the $\overline{\mathrm{MS}}$ scheme predicted by the D-log Padés to the Borel transform of the reduced second derivative in QCD. . . . . . . 104

Table 7 - Coefficients $r_{n}$ in the $\overline{\mathrm{MS}}$ scheme predicted by the Padés to the perturbative series in $a_{s}$ of the reduced second derivative in QCD. . . 105

Table 8 - Coefficients $d_{n, 1}$ in the $\overline{\mathrm{MS}}$ scheme predicted by Padés and D-log Padés to the second derivative in QCD. . . . . . . . . . 106

Table 9 - Final values for the coefficients $d_{n, 1}$ in $\overline{\mathrm{MS}}$ obtained from the Padé approximants of Tab. 8 in QCD. . . . . . . . . . . 106

Table 10 - Final values for the coefficients $c_{n}$ of the imaginary part of the scalar correlator in QCD obtained from the results in Tab. 9. . . . . . . 107 



\section{CONTENTS}

INTRODUCTION . . . . . . . . . . . . . 19

STANDARD MODEL . . . . . . . . . . . . . 23

$2.1 \quad$ SM Lagrangian . . . . . . . . . . . . . . . . . . . . 24

2.1.1 The Electroweak Lagrangian . . . . . . . . . . . . . . . . . 24

2.1.2 Higgs Mechanism . . . . . . . . . . . . . . . . . 26

2.1.3 The QCD Lagrangian . . . . . . . . . . . . . . . . . 29

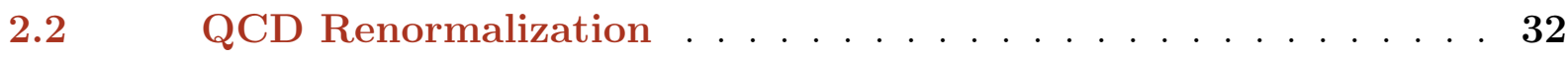

2.3 Running Coupling Constant and Running Quark Mass . . . . . . 35

3 QCD IN THE DECAY OF THE HIGGS INTO BOTTOM QUARKS ................... 41

3.1 Leading-Order Term . . . . . . . . . . . . . . . 41

3.2 Master Integrals Method . . . . . . . . . . . . . . . . 45

3.2.1 One-Loop Massless Propagator . . . . . . . . . . . . . . . . . . . 45

3.2.2 Two-Loop Massless Propagator . . . . . . . . . . . . . . 47

3.2.3 $H \rightarrow b \bar{b}$ at Next-to-Leading Order . . . . . . . . . . . . . . 49

3.3 Scalar Correlator at Higher Orders . . . . . . . . . . . . . 53

4 ASYMPTOTIC SERIES AND PADÉ APPROXIMANTS • . 57

$4.1 \quad$ Divergent Series and Renormalons . . . . . . . . . . 57

$4.2 \quad$ Asymptotic Series and Borel Transform . . . . . . . . . . . 59

$4.3 \quad$ Padé Approximants . . . . . . . . . . . . . . 63

$5 \quad$ LARGE- $\beta_{0}$ LIMIT . . . . . . . . . . . . . . 71

$5.1 \quad$ Scalar Correlator and Renormalons in the Large- $\boldsymbol{\beta}_{0}$ Limit . . . . 72

$5.1 .1 \quad$ Scheme Variation . . . . . . . . . . . . . . 79

5.2 Padé Approximants to the Imaginary Part in the Large- $\boldsymbol{\beta}_{0} \ldots \quad$. 82

$5.2 .1 \quad$ Scheme Variation . . . . . . . . . . . . . . . . . . . 84

5.2.2 Partial Padé Approximants to the Imaginary Part in the Large- $\beta_{0}$. . . 87

5.3 Padé Approximants to the Second Derivative in the Large- $\boldsymbol{\beta}_{0} \quad$. 90

5.3.1 D-log Padés to the Second Derivative in the Large- $\beta_{0} \ldots \ldots$. . . . . . 92

5.4 Calculating the Coefficients $d_{n, 1} \ldots \ldots \ldots \ldots$. . . . . . . 94

5.5 Partial Conclusions . . . . . . . . . . . . . . 97

$6 \quad$ RESULTS IN QCD . . . . . . . . . . . . 99

6.1 Results for the Imaginary Part in QCD . . . . . . . . . . 99 
6.2 Results for the Second Derivative in QCD . . . . . . . . . 101

6.2.1 D- $\log$ Padés to the Second Derivative in QCD . . . . . . . . . . . 103

6.2.2 Padés to the Series in $a_{s}$ in QCD . . . . . . . . . . . . . . 104

$6.3 \quad$ Final Results . . . . . . . . . . . . . . . . 105

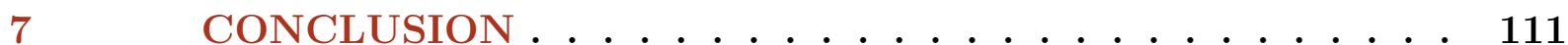

REFERENCES ................... 115 


\section{INTRODUCTION}

The Standard Model (SM) was developed in the early 1970s and is the non-abelian theory that describes the fundamental particles, fermions and bosons, and their electromagnetic, strong and weak interactions. The fermions interact with each other by the exchange of gauge bosons with spin 1, where each interaction is mediated by a different boson. In the SM there is still a scalar boson denominated Higgs that was proposed in 1964 by Robert Brout, François Englert ${ }^{1}$ and Peter Higgs, ${ }^{2}$ among others. This boson appears in the Higgs mechanism, that is responsible for the quark mixing and generates mass for the bosons $W$ and $Z$ and the fermions, which are not allowed because of local gauge invariance.

The SM can be divided in two non-abelian gauge theories: Quantum Chromodynamics (QCD), which will be fundamental in this dissertation, and the electroweak (EW) sector, that describes the electromagnetic interaction, mediated by the photon, as well as the weak interaction, mediated by the bosons $W^{ \pm}$and $Z$. QCD explains the strong interactions, where the quarks, fermionic particles, interact through the exchange of gluons, the gauge boson of this theory. The gluons also interact with other gluons due to the $S U(3)_{c}$ symmetry of QCD. All the interactions of this theory have their intensities proportional to powers of the strong coupling of QCD, $\alpha_{s}$.

The Standard Model explains successfully a great part of the experimental results and accurately predicts a wide variety of phenomena, becoming one of the most tested theories in physics. One of its greatest triumphs was the detection of a particle, announced on July $4^{\text {th }} 2012$, by the ATLAS ${ }^{3}$ and CMS ${ }^{4}$ experiments of the CERN's Large Hadron Collider (LHC). The particle discovered has a mass of approximately $125 \mathrm{GeV}$ and its quantum numbers and properties are consistent with the ones predicted by the SM for the Higgs boson. Due to this discovery, in 2013 François Englert and Peter Higgs were awarded the Nobel prize in Physics.

Although the SM is well tested, there are still some unexplained phenomena, for example the neutrino masses and the observed matter-antimatter asymmetry, which indicates that the Standard Model should not be the final theory. However, no direct observation of new physics was done so far: since the discovery of the Higgs boson no new particles were detected. Then, it is possible to search for physics beyond the Standard Model through precision physics, and, for the Higgs sector, an observable that has received a lot of attention are the signal strengths denoted $\mu$. They consist in the ratio between the cross section of Higgs production and decay in a given channel measured experimentally and the theoretical predictions within the SM. Hence, for the Higgs predicted by the 
Standard Model, we should have $\mu$ compatible with 1 . The latest results, with the analysis of the CMS data, for the weighted average of the signal strength for different channels is $\bar{\mu}=1.17 \pm 0.10,{ }^{5}$ which is in agreement with the SM.

In order to detect deviations in the values of the signal strengths, it is necessary to increase the precision of both theoretical and experimental values. Thus, the Higgs decay rates and cross sections need to be calculated at even higher orders in perturbation theory. The decay width of the Higgs boson is dominated by the channel $H \rightarrow b \bar{b}$ and in processes involving quarks and gluons, the QCD corrections are dominant compared to the electroweak ones. Since 2006 this decay rate, $\Gamma(H \rightarrow b \bar{b})$, is know up to fourth order in the strong coupling $\left(\alpha_{s}^{4}\right)$ in massless perturbative QCD. ${ }^{6,7}$ It is extremely difficult to obtain the corrections to the perturbative series, which is corroborated by the interval between the computations of the third and fourth-order coefficients: 10 years. ${ }^{6,8}$ Since there is no indication that the higher-order terms will be calculated exactly any time soon, it is important to employ other methods to estimate them with the available information, which is what we will do in this dissertation.

The perturbative series of the decay rate of the Higgs boson into bottom quarks is expected to be asymptotic, i.e., when truncated at intermediate orders gives a good approximation, with factorially divergent coefficients. The divergent behavior of the series is governed by the renormalons, singularities on the real axis of the Borel transform of the series, which is essentially its inverse Laplace transform - a widely used technique to "sum" asymptotic series. The position of theses singularities are known since they are related to the dimension of the operators participating in the Operator Product Expansion (OPE) of the correlator. ${ }^{9,10}$

Since the Borel transform has singularities, the renormalons, we can use rational approximants to model the series. Thus we will use Padé approximants ${ }^{11-13}$ allied to the Borel transform to reconstruct the perturbative series of the decay rate to all orders. These rational approximants can be made model independently and in some cases there are theorems that state their convergence to the original function. The Padé approximants have been used in perturbative series of quantum field theories to determine unknown higher-order coefficients since the $90 \mathrm{~s} .{ }^{14,15}$ A similar method to what will be applied in this dissertation, employing the Borel transform of the series, a procedure sometimes called Padé-Borel method, ${ }^{16}$ was also tested in that decade for various QCD processes. ${ }^{17} \mathrm{~A}$ similar technique was also employed very recently in the study of the hadronic $\tau$ decays. ${ }^{18}$

In order to assess the quality of this approach, before we apply the method in the perturbative series in QCD, we will apply it in the so-called large- $\beta_{0}$ limit. In this limit all the contributions due to the insertion of fermion bubble chains in the gluon propagator are calculated. ${ }^{19}$ Each fermion loop contributes to the dressed gluon propagator with a factor of $\beta_{0 f}$, which is the fermionic term of the one-loop $\beta$ function and is proportional to $N_{f}$, the number of quark flavors. To obtain the large- $\beta_{0}$ limit, that will be better discussed 
later, we have to perform a process denominated non-abelianization, which consists in substituting $\beta_{0 f}$ by $\frac{\beta_{1}}{2 \pi}$. For the Higgs decay into bottom quarks the series in this limit is known up to all orders,${ }^{20}$ thus it is possible to determine the most efficient strategy in large- $\beta_{0}$, which will guide the best method to employ in full QCD. In addition, the Borel transform of this series has an infinite number of poles on the real axis, the aforementioned renormalons of perturbation theory, hence it is a meromorphic function and the PA theory to meromorphic functions apply, specially Pomerenke's theorem. ${ }^{11}$

We will, besides the standard Padés, consider other approximants and techniques to accelerate the convergence. We will employ partial Padé approximants, which use the available knowledge about the analytical structure of the function, and the D-log Padés, which are not, strictly speaking, rational approximants although they can be efficient to approximate functions with branch cuts. Furthermore, the dependence of the perturbative series on the renormalization scheme will also be analyzed through the parametrization of the dependence of the strong coupling on the renormalization scheme by a parameter $C$, suggested recently by Boito, Jamin and Miravitllas. ${ }^{21}$ Then a different value for this parameter is related to a different renormalization scheme, which can be used to improve the convergence of the approximants.

With the systematic study of the method of Padé approximants in the large- $\beta_{0}$ limit, we can direct the strategies that will be applied to the perturbative series in full QCD, where only the first four coefficients are known. The characteristics of the Borel transform in QCD are different, since now the poles turn into branch cuts. ${ }^{9}$ There are no convergence theorem in this case, although practical examples show that the convergence occurs. ${ }^{18}$ We will apply the Padé-Borel method to the series in QCD of the decay rate of Higgs into bottom quarks in order to estimate the fifth-order coefficient and its Borel sum. Despite the fact that the renormalon structure of QCD is not fully understood, there is an indication that the IR renormalons govern the behavior of the perturbative series at lower and intermediate orders, which causes the absence of the sign-alternating behavior at these orders.

This dissertation is organized in the following way: in Ch. 2 we present a review of the Standard Model lagrangian and some fundamental aspects of QCD. In Ch. 3 we describe the Higgs decay into a pair of bottom quarks and calculate the first-order correction to the decay rate. In Ch. 4 we introduce the origin of the renormalons and the mathematical theory for asymptotic series, Borel transforms and Padé approximants. In Ch. 5 we discuss some aspects of the perturbative series in the large- $\beta_{0}$ limit and the application of Padé approximants in this limit. In Ch. 6 the results obtained from the use of Padé approximants in full QCD are shown. And finally in Ch. 7 we present our final conclusions. 



\section{STANDARD MODEL}

The Standard Model (SM) is a gauge theory based on the symmetry group $S U(3)_{c} \otimes$ $S U(2)_{L} \otimes U(1)_{Y}$ that describes three of the fundamental forces: electromagnetic, weak and strong interactions. Here we will present a brief description of the matter and symmetry content of the SM. In the next sections we discuss the main aspects of Quantum Chromodynamics that will be the basis for the calculations of the corrections to the decay width of the Higgs boson into a $b \bar{b}$ pair in our dissertation.

Within the SM, the matter is made of the fermions, which are elementary particles. Each fermion has its antiparticle, which has the same mass but opposite quantum numbers. The fermions have spin $1 / 2$ and are classified in leptons and quarks. The leptons have six different varieties (known as flavors): electron, muon and tau, which are massive and carry charge -1 , and their associated neutrinos $\left(\nu_{e}, \nu_{\mu}, \nu_{\tau}\right)$ that are massless particles in the Standard Model*.

The quarks also have six flavors, up, down, strange, charm, bottom and top and have fractional electric charge: up, charm and top have charge $2 / 3$ (in unities of the proton charge), down, strange and bottom have charge $-1 / 3$. They also have another quantum number denominated color where each quark can have three different colors often referred to as red, green and blue. The quarks are the constituents of the hadrons, that are divided in two subgroups: the baryons, which have half-integer spin and are composed by three quarks, $q q q$, as neutrons and protons; and the mesons, which have integer spin and are composed by a quark and an antiquark, $q \bar{q}$, as pions. Since colored states were never observed, it is necessary then to postulate that all asymptotic states are colorless, in other words, singlets under rotations in the color space. This is known as the confinement hypothesis, since it implicates that free quarks cannot be observed.

It is customary to organize the fermions in generations as shown below

$$
\left[\begin{array}{ll}
\nu_{e} & u \\
e^{-} & d
\end{array}\right], \quad\left[\begin{array}{ll}
\nu_{\mu} & s \\
\mu^{-} & c
\end{array}\right], \quad\left[\begin{array}{ll}
\nu_{\tau} & b \\
\tau^{-} & t
\end{array}\right] .
$$

The first generation is composed by the lightest particles, whereas the less-stable and heavier ones constitute the other two generations. These families only differ by mass and flavor. The ordinary matter in the universe is made of particles belonging to the first generation, since the other ones decay to more stable particles.

In this theory, the interactions between fermions are mediated by the exchange of bosons of spin 1. Each interaction is mediated by its own boson: a massless photon for the

\footnotetext{
${ }^{*}$ Although in the SM the neutrinos are considered massless, it was observed that they oscillate between different flavors. ${ }^{22}$ This phenomenon indicates that neutrinos are massive.
} 
electromagnetic interaction, eight massless gluons for the strong one and three massive bosons, $W^{ \pm}$and $Z$, for the weak interaction.

Beyond the fermions and the spin 1 bosons, there is also the scalar Higgs boson which was first proposed in 1964 by Robert Brout, François Englert ${ }^{1}$ and Peter Higgs ${ }^{2}$ as a mechanism to generate mass to the $W$ and $Z$ bosons and the fermions, which is not allowed due to local gauge invariance. In this mechanism (that will be discussed in more detail in Sec. 2.1.2), the gauge symmetry is broken by the vacuum (lowest energy state), which triggers the Spontaneous Symmetry Breaking (SSB) that causes the breaking of the SM gauge group following the pattern $S U(3)_{c} \otimes S U(2)_{L} \otimes U(1)_{Y} \rightarrow S U(3)_{c} \otimes U(1)_{\text {QED }}$. This particle was only observed in 2012 by $\mathrm{ATLAS}^{3}$ and $\mathrm{CMS}^{4}$ experiments in LHC, which led to the Nobel prize being awarded to Englert and Higgs in 2013.

\subsection{SM Lagrangian}

We start with a discussion of the SM lagrangian, since it generates the Feynman rules, which are the fundamental tools to obtaining the decay width of the Higgs boson into $b \bar{b}$. The symmetry group of the Standard Model can be divided in two subgroups:

* $S U(3)_{c}$, which leads to Quantum Chromodynamics (QCD), a non-abelian gauge theory that describes the strong interactions between quarks mediated by gluons;

* $S U(2)_{L} \otimes U(1)_{Y}$, that gives rise to the electroweak (EW) sector, which includes the electromagnetic interactions, mediated by the photon, and the weak interactions, mediated by the bosons $W^{ \pm}$and $Z$.

We will analyze now each lagrangian separately where the main reference adopted in this part of the dissertation is the review of Pich. ${ }^{23}$

\subsubsection{The Electroweak Lagrangian}

The EW theory does not allow masses for the bosons $W$ and $Z$, which have to be massive due to the short range interactions they mediate which are manifest, for example, in the nuclear beta decay. The need for these bosons to be massive was the motivation for the development of the Higgs mechanism in the 60s.

The electroweak sector is a Yang-Mills field theory invariant under the non-abelian group $S U(2)_{L} \otimes U(1)_{Y}$, where $L$ stands for left-handed fields and $Y$ indicates hypercharge. The EW theory is said to be chiral, i.e., the left- and right-handed fermions behave differently under the gauge group. That occurs because the left-handed fields are doublets under $S U(2)$ and the right-handed ones are singlets.

In order to obtain the lagrangian, one needs first to introduce the notation below for the first family of quarks 


$$
\psi_{1}(x)=\left(\begin{array}{l}
u \\
d
\end{array}\right)_{L}, \quad \psi_{2}(x)=u_{R}, \quad \psi_{3}(x)=d_{R} .
$$

The same notation can be used for the leptons and the other families indicated in Eq. (2.1)

$$
\psi_{1}(x)=\left(\begin{array}{c}
\nu_{e} \\
e^{-}
\end{array}\right)_{L}, \quad \psi_{2}(x)=\nu_{e R}, \quad \psi_{3}(x)=e_{R}^{-} .
$$

The lagrangian can be obtained by requiring that the free-fermion lagrangian should be invariant under local $S U(2)_{L} \otimes U(1)_{Y}$ transformations and can be written as

$$
\mathscr{L}_{\mathrm{EW}}=-\frac{1}{4} B_{\mu \nu} B^{\mu \nu}-\frac{1}{4} W_{\mu \nu}^{i} W^{\mu \nu i}+i \bar{\psi}_{j} \gamma_{\mu} D^{\mu} \psi_{j}
$$

where the field strength tensors are given by

$$
B_{\mu \nu}=\partial_{\mu} B_{\nu}-\partial_{\nu} B_{\mu}, \quad W_{\mu \nu}^{i}=\partial_{\mu} W_{\nu}^{i}-\partial_{\nu} W_{\mu}^{i}-g \epsilon^{i j k} W_{\mu}^{j} W_{\nu}^{k},
$$

and the covariant derivatives are

$$
\begin{aligned}
& D_{\mu} \psi_{1}=\left(\partial_{\mu}+i g \frac{\sigma^{i}}{2} W_{\mu}^{i}+i g^{\prime} Y_{1} B_{\mu}\right) \psi_{1}, \\
& D_{\mu} \psi_{j}=\left(\partial_{\mu}+i g^{\prime} Y_{j} B_{\mu}\right) \psi_{j} \quad j=2,3 .
\end{aligned}
$$

The derivative acts differently on the right-handed fields because they are a singlet under $S U(2)$ hence they do not couple with the $W$ bosons. The symbol $\epsilon^{i j k}$ is the Levi-Civita tensor, $g$ the $S U(2)$ gauge coupling, $g^{\prime}$ is the $U(1)$ coupling, $W_{\mu}^{i}(i=1,2,3)$ are the $S U(2)$ gauge fields and $B_{\mu}$ is the $U(1)$ gauge field associated with the hypercharge $Y$. A mass term for both the gauge bosons and the fermions cannot be included in the lagrangian in Eq. (2.4) because it would violate the local gauge symmetry.

The first two terms of the lagrangian in Eq. (2.4) are the kinetic terms for the fields $W_{\mu}^{i}$ and $B_{\mu}$, that generate their free propagators. It also gives rise to the cubic and quartic self-interactions of $W_{\mu}^{i}$ fields, since their field strength have a quadratic term, which is proportional to the coupling $g$ of $S U(2)$. In addition, the last term of Eq. (2.4) includes the kinetic term for the fermions and the interactions between them and the gauge bosons.

The part that contains the $W_{\mu}^{i}$ field generates the charged-current interactions. Defining the fields $W_{\mu}^{ \pm}$, which represent the bosons $W^{ \pm}$, as

$$
W_{\mu}^{ \pm}=\frac{1}{\sqrt{2}}\left(W_{\mu}^{1} \mp i W_{\mu}^{2}\right)
$$

one can write for the first generation the lagrangian below

$$
\mathscr{L}_{c c}=-\frac{g}{2 \sqrt{2}}\left\{W_{\mu}^{+}\left[\bar{u}_{L} \gamma^{\mu}\left(1-\gamma_{5}\right) d_{L}+\bar{\nu}_{e L} \gamma^{\mu}\left(1-\gamma_{5}\right) e_{L}\right]+\text { h.c. }\right\} .
$$

From this lagrangian one can calculate the coupling between fermions and the bosons $W^{ \pm}$. 
It is possible to notice that the charged-current interaction permits flavor changing, and indeed is the only interaction in the SM that allows it.

The last term of the lagrangian in Eq. (2.4) also leads to the interactions with the neutral gauge fields $W_{\mu}^{3}$ and $B_{\mu}$, responsible for the neutral weak and electromagnetic interactions. Those fields can be written as a combination of $A_{\mu}$ and $Z_{\mu}$

$$
W_{\mu}^{3}=\cos \theta_{W} Z_{\mu}+\sin \theta_{W} A_{\mu}, \quad B_{\mu}=-\sin \theta_{W} Z_{\mu}+\cos \theta_{W} A_{\mu},
$$

where $\theta_{W}$ is the weak mixing angle, $A_{\mu}$ and $Z_{\mu}$ represent the photon and $Z$ boson respectively. In order to identify $A_{\mu}$ as the photon field, it is necessary to impose the following conditions

$$
g \sin \theta_{W}=g^{\prime} \cos \theta_{W}=e \quad \text { and } \quad Y=Q-T_{3},
$$

with $T_{3}$ and $Q$ being respectively the weak isospin and electromagnetic charge operator. One can write now the interaction lagrangian below

$$
\mathscr{L}_{n c}=-e A_{\mu} \bar{\psi}_{j} \gamma^{\mu} Q_{j} \psi_{j}-\frac{e}{\sin \theta_{W} \cos \theta_{W}} Z_{\mu} \bar{\psi}_{j}\left(T_{3}-Q_{j} \sin ^{2} \theta_{W}\right) \psi_{j}
$$

The first part is the usual QED lagrangian and the last one contains the interaction with the $Z$ boson. Using the equation above it is possible to determine the coupling between fermions and the gauge bosons, the photon and the $Z$ boson.

It is important to notice that the local gauge symmetry forbids a mass term for both gauge bosons and fermions. However, since the interactions mediated by the bosons $W^{ \pm}$ and $Z$ are short ranged, they have to be massive. The masses for these particles will emerge from the Higgs mechanism.

\subsubsection{Higgs Mechanism}

Before we analyze the Higgs mechanism in the SM, we will first consider a simpler case, a complex scalar field $\phi$ with the lagrangian given below

$$
\mathscr{L}=\frac{1}{2} \partial_{\mu} \phi^{*} \partial^{\mu} \phi-V(\phi), \quad V(\phi)=\mu^{2} \phi^{*} \phi+\lambda\left(\phi^{*} \phi\right)^{2} .
$$

This lagrangian is invariant under global phase transformations of the scalar field $\phi \rightarrow$ $\phi^{\prime}=\mathrm{e}^{i \alpha} \phi$. For the potential to have a finite minimum, the parameter $\lambda$ must be positive. If the term $\mu^{2}$ is also positive, the potential, that is shown in Fig. 1a, has a minimum at $\langle 0|\phi| 0\rangle \equiv \phi_{0}=0$. In this case, the lagrangian in Eq. (2.13) represents a scalar particle with mass $\mu \sqrt{2}$. On the other hand, if we consider $\mu^{2}$ negative, the potential has a non-zero minimum when

$$
\phi^{*} \phi=|\phi|^{2}=-\frac{\mu^{2}}{2 \lambda} \equiv \frac{v^{2}}{2}
$$

as shown in Fig. 1b. This quantity is called the vacuum expectation value (vev) of the 


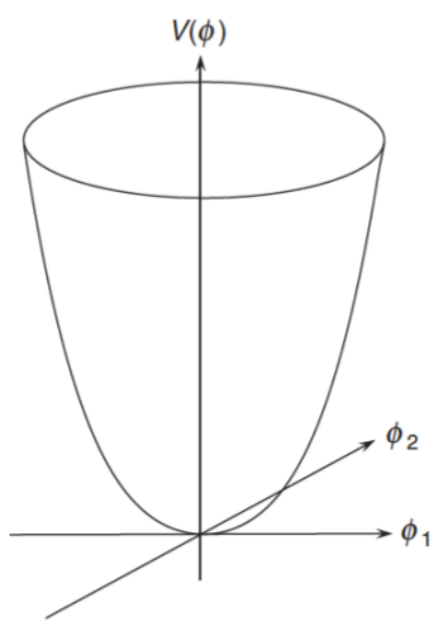

(a)

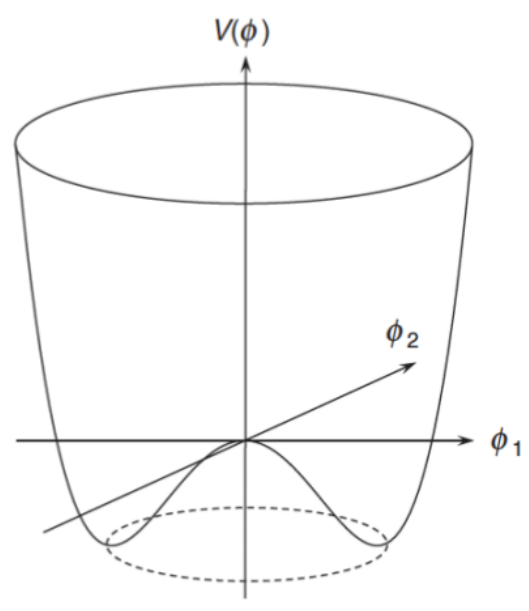

(b)

Figure 1 - The potential $V(\phi)$ for a complex scalar field for (a) $\mu^{2}>0$ and (b) $\mu^{2}<0$.

Source: THOMSON. ${ }^{24}$

field $\phi$. There is now, due to $U(1)$ symmetry of the lagrangian, an infinite number of degenerate states of minimum energy $\phi=v \mathrm{e}^{i \alpha}$. By choosing a particular solution as the vev, the symmetry is broken, a process known as Spontaneous Symmetry Breaking. In this condition, to understand correctly the theory, we have to parametrize the excitations over the vacuum states. Without loss of generality, we can choose the field to be

$$
\phi=\frac{1}{\sqrt{2}}\left(v+\phi_{1}+i \phi_{2}\right)
$$

where the fields $\phi_{i}$ are real. In terms of the fields $\phi_{i}$, the lagrangian can be rewritten as

$$
\mathscr{L}=\frac{1}{2} \partial_{\mu} \phi_{1} \partial^{\mu} \phi_{1}-\lambda v^{2} \phi_{1}^{2}+\frac{1}{2} \partial_{\mu} \phi_{2} \partial^{\mu} \phi_{2}-V_{\text {int }},
$$

where $V_{\text {int }}$ contains the interaction terms

$$
V_{\mathrm{int}}=\lambda v \phi_{1}^{3}+\frac{1}{4} \lambda \phi_{1}^{4}+\frac{1}{4} \lambda \phi_{2}^{4}+\frac{1}{2} \lambda \phi_{1}^{2} \phi_{2}^{2}+\lambda v \phi_{1} \phi_{2}^{2}
$$

One can see that this lagrangian represents a massive scalar field $\phi_{1}$ whose mass is $m^{2}=2 \lambda v^{2}=-2 \mu^{2}$ and a massless scalar field $\phi_{2}$.

This brings us to the Goldstone theorem. ${ }^{25-27}$ This theorem states that spontaneous breaking of continuous global symmetries implies the existence of massless particles, known as Goldstone bosons, in a number equal to the broken generators. In this example, the symmetry $U(1)$ is broken, due to our choice of a particular point on the circle to expand.

Let us now turn to the Higgs mechanism in the SM, that is a non-abelian theory. We need to generate mass to three gauge bosons, and this requires at least three degrees of freedom. The simplest choice is an $S U(2)$ doublet $\Phi$ of scalar fields $\phi$ 


$$
\Phi=\left(\begin{array}{c}
\phi^{+} \\
\phi^{0}
\end{array}\right)
$$

where one of them must be neutral $\left(\phi^{0}\right)$ and the other must be charged $\left(\phi^{+}\right)$. The lagrangian to this doublet is given by

$$
\mathscr{L}=\left(D^{\mu} \Phi\right)^{\dagger}\left(D_{\mu} \Phi\right)-\mu^{2} \Phi^{\dagger} \Phi-\lambda\left(\Phi^{\dagger} \Phi\right)^{2}
$$

where $D_{\mu}$ is the covariant derivative shown in Eq. (2.6) so the Lagrangian of $\Phi$ respects the $S U(2)_{L} \otimes U(1)_{Y}$ local gauge symmetry that the electroweak sector of the SM has.

As before, for $\mu^{2}$ negative, there is an infinite number of degenerate states with minimum energy that satisfies

$$
\left|\Phi^{\dagger} \Phi\right|=\sqrt{\frac{-\mu^{2}}{2 \lambda}} \equiv \frac{v}{\sqrt{2}} .
$$

The charged field cannot acquire a vev because the electric charge is a conserved quantity. After choosing a particular vev, the $S U(2)_{L} \otimes U(1)_{Y}$ symmetry breaks spontaneously to $U(1)_{\text {QED }}$. Then, according to the Goldstone theorem, three massless bosons should appear, since the broken group $S U(2)$ has three generators.

Now, we can parametrize the scalar doublet in terms of four real fields, $\theta_{i}$ and $H$, in the general form

$$
\Phi=\frac{1}{\sqrt{2}} \exp \left[i \frac{\sigma_{a}}{2} \theta^{a}\right]\left(\begin{array}{c}
0 \\
v+H
\end{array}\right) .
$$

Because of the local $S U(2)_{L}$ invariance, a gauge transformation on $\Phi$ can be done to move it to the so-called unitary gauge, where it is entirely real and written as

$$
\Phi=\frac{1}{\sqrt{2}}\left(\begin{array}{c}
0 \\
v+H
\end{array}\right) .
$$

It is necessary to identify the masses of the gauge bosons. It can be done by expanding the covariant derivative that acts in $\Phi$

$$
\left(D^{\mu} \Phi\right)^{\dagger}\left(D_{\mu} \Phi\right)=\frac{1}{2} \partial_{\mu} H \partial^{\mu} H+\frac{1}{8} g^{2}(v+H)^{2} W_{\mu}^{-} W^{+\mu}+\frac{g^{2}}{8 \cos ^{2} \theta_{W}}(v+H)^{2} Z_{\mu} Z^{\mu},
$$

where we defined the fields as in Eqs. (2.8) and (2.10). It is possible to notice that, as expected, the bosons $W^{ \pm}$and $Z$ acquire mass

$$
m_{W}=\frac{1}{2} v g, \quad m_{Z}=\frac{g v}{2 \cos \theta_{W}}=\frac{v}{2} \sqrt{g^{2}+g^{\prime 2}},
$$

and the photon is still massless, as it should be, because the $U(1)$ symmetry is unbroken. From the expression in Eq. (2.23) one can also determine the vertices of the interactions between the Higgs and $W^{ \pm} / Z$ bosons. 


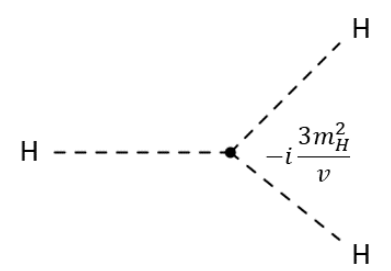

Figure 2 - 3-Higgs vertex.

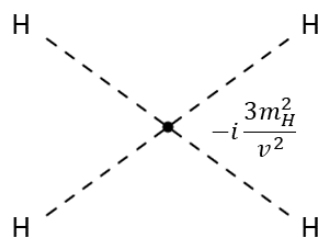

Figure 3 - 4-Higgs vertex.

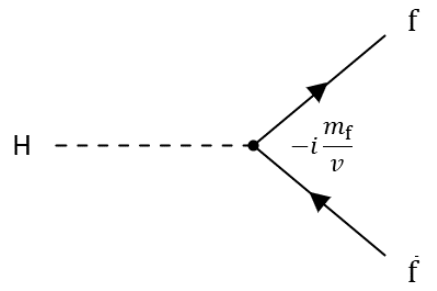

Figure 4 - Interaction vertex between Higgs and fermions.

Source: By the author.

Now expanding the potential of the scalar doublet we find

$$
V=\lambda v^{2} H^{2}+\lambda v H^{3}+\frac{1}{4} \lambda H^{4} .
$$

The first term gives the mass of the Higgs boson: $m_{H}=\sqrt{2 \lambda v^{2}}=\sqrt{-2 \mu^{2}}$, and the other ones give the Higgs self-couplings. There are two possible vertices: ${ }^{28}$ the first one, shown in Fig. 2, is an interaction vertex of three Higgs bosons, given by $-i \frac{3 m_{H}^{2}}{v}$; the other one is an interaction vertex of four Higgs bosons, illustrated in Fig. 3, given by $-i \frac{3 m_{H}^{2}}{v^{2}}$.

Additionally, this doublet can also be used to give mass to the fermions, since it is possible now to write a $S U(2)_{L} \otimes U(1)_{Y}$ invariant lagrangian. This lagrangian is denominated Yukawa lagrangian and for the first generation is given by

$$
\mathscr{L}_{Y}=-y_{d}\left(\begin{array}{ll}
\bar{u} & \bar{d}
\end{array}\right)_{L} \Phi d_{R}-y_{u}\left(\begin{array}{ll}
\bar{u} & \bar{d}
\end{array}\right)_{L} \Phi^{c} u_{R}-y_{e}\left(\begin{array}{ll}
\bar{v}_{e} & \bar{e}
\end{array}\right)_{L} \Phi e_{R}-y_{e} \bar{e}_{R} \Phi^{\dagger}\left(\begin{array}{c}
\bar{v}_{e} \\
\bar{e}
\end{array}\right)_{L}
$$

where $\Phi^{c} \equiv i \sigma_{2} \Phi^{*}$ is the charge conjugate of $\Phi$. Using the field $\Phi$ in the unitary gauge as given in Eq. (2.22), one finds that

$$
\mathscr{L}_{Y}=-\frac{1}{\sqrt{2}}(v+H) \sum_{f} y_{f} \bar{f} f,
$$

where $f$ represents the fermion field. Note that now the fermions acquire a mass equals to $m_{f}=\frac{1}{\sqrt{2}} y_{f} v$. They can also interact with the Higgs boson, whose vertex is displayed in Fig. 4 and is given by $-i \frac{m_{f}}{v}$, which is proportional to the mass of the fermion. This is the essential interaction of this dissertation, since we are studying the decay $H \rightarrow b \bar{b}$. This interaction is the one responsible for the tree-level decay of Higgs into $b$ quarks, which will be analyzed in Ch. 3.

\subsubsection{The QCD Lagrangian}

We will turn now to QCD, which is a Yang-Mills quantum field theory invariant under the non-abelian group $S U(3)_{c}$, where $c$ refers to color. We will analyze the QCD lagrangian 
outside of the context of the Standard Model, so it is possible to write a mass term for the quarks without violating any symmetry. In the SM this term requires the Higgs boson as explained above. The QCD lagrangian can be determined by imposing that the lagrangian of free quarks is invariant under local $S U(3)_{c}$ transformations resulting in

$$
\mathscr{L}_{\mathrm{QCD}} \equiv-\frac{1}{4} G_{a}^{\mu \nu} G_{\mu \nu}^{a}+\sum_{f} \bar{q}_{f}\left(i \gamma^{\mu} D_{\mu}-m_{f}\right) q_{f}
$$

where $f$ stands for the quark flavor, $m_{f}$ is the quark mass, $q_{f}$ is the color triplet,

$$
G_{a}^{\mu \nu}=\partial^{\mu} G_{a}^{\nu}-\partial^{\nu} G_{a}^{\mu}+g_{s} f^{a b c} G_{b}^{\mu} G_{c}^{\nu} \quad \text { and } \quad D_{\mu} \equiv \partial_{\mu}-i g_{s} \frac{\lambda^{a}}{2} G_{a}^{\mu} .
$$

The gluon field is represented by $G_{a}^{\mu}$, where $a$ is the color; $g_{s}$ is the coupling of the theory and $\lambda^{a}$ are the generators of the fundamental representation of the $S U(3)_{c}$ algebra which satisfies the commutation relation below

$$
\left[\lambda^{a}, \lambda^{b}\right]=2 i f^{a b c} \lambda^{c}
$$

with $f^{a b c}$ being the $S U(3)_{c}$ structure constants. The $S U(3)$ gauge symmetry forbids the addition of a mass term for the gluon fields, although the quark mass is allowed. One can now decompose the lagrangian in the following way

$$
\begin{aligned}
\mathscr{L}_{\mathrm{QCD}}= & -\frac{1}{4}\left(\partial^{\mu} G_{a}^{\nu}-\partial^{\nu} G_{a}^{\mu}\right)\left(\partial_{\mu} G_{\nu}^{a}-\partial_{\nu} G_{\mu}^{a}\right)+\sum_{f} \bar{q}_{f}^{\alpha}\left(i \gamma^{\mu} \partial_{\mu}-m_{f}\right) q_{f}^{\alpha}+ \\
& +g_{s} G_{a}^{\mu} \sum_{f} \bar{q}_{f}^{\alpha} \gamma_{\mu}\left(\frac{\lambda^{a}}{2}\right)_{\alpha \beta} q_{f}^{\beta}- \\
& -\frac{g_{s}}{2} f^{a b c}\left(\partial^{\mu} G_{a}^{\nu}-\partial^{\nu} G_{a}^{\mu}\right) G_{\mu}^{b} G_{\nu}^{c}-\frac{g_{s}^{2}}{4} f^{a b c} f_{a d e} G_{b}^{\mu} G_{c}^{\nu} G_{\mu}^{d} G_{\nu}^{e}
\end{aligned}
$$

where $q_{f}^{\alpha}$ is the quark field of color $\alpha$. The indices $\alpha$ and $\beta$ run from 1 to 3 , and the indices $a, b, c, d$ and $e$ run from 1 to 8 .

The first line of Eq. (2.31) gives the kinetic terms for the quark and gluon fields, which generate the free propagators, and the second line contains the color interaction between quarks and gluons. Due to the non-abelian character of $S U(3)_{c}$, i.e., the generators $\lambda^{a}$ do not commute, the term $G_{a}^{\mu \nu} G_{\mu \nu}^{a}$ results in the cubic and quartic gluon self-interactions present in the third line of Eq. (2.31). All the interaction terms are proportional to powers of the strong coupling constant $g_{s}$, thus the coupling is universal in QCD.

One can now quantize the QCD lagrangian by quantizing the quark and gluon fields. Within canonic quantization, this is achieved by writing a generic spinor field $\psi(x)$ (omitting color indices and polarization) in terms of creation and annihilation operators as

$$
\psi(x)=\int \frac{\mathrm{d}^{3} p}{(2 \pi)^{3}} \frac{1}{\sqrt{2 E(\vec{p})}}\left[a_{s}(\vec{p}) u_{s}(\vec{p}) \mathrm{e}^{-i p x}+b_{s}^{\dagger}(\vec{p}) v_{s}(\vec{p}) \mathrm{e}^{-i p x}\right],
$$

with the four-spinors $u_{s}(\vec{p})$ and $v_{s}(\vec{p})$ being the solutions of the free Dirac equation in 


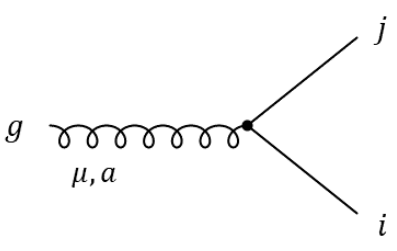

Figure 5 - Interaction vertex between gluons and quarks.

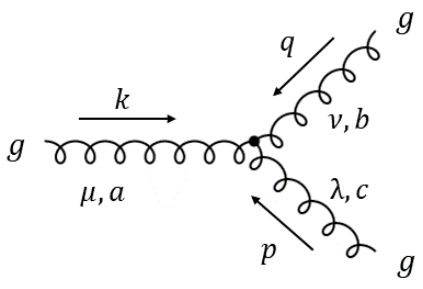

Figure 6 - 3-gluon vertex.

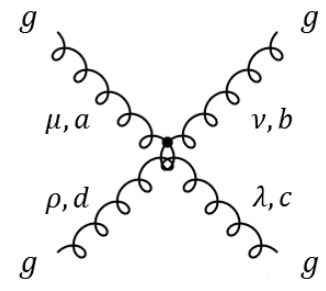

Figure 7 - 4-gluon vertex.

Source: By the author.

momentum space and $s$ the helicity state of the spinor. Applying the commutation relations for the annihilation and creation operators we can demonstrate that the Feynman propagator for the free spinor field is ${ }^{29}$

$$
i S(x-y)=\langle 0|T[\psi(x) \bar{\psi}(y)]| 0\rangle=i \int \frac{\mathrm{d}^{4} p}{(2 \pi)^{4}} \frac{\not p+m}{p^{2}-m^{2}+i 0} \mathrm{e}^{-i p(x-y)} .
$$

$T$ represents the time-ordering operator and the term $i 0$ is the Feynman prescription of the propagator and it maintains causality. The propagator for the quarks in momentum space is then

$$
i S(p)=i \frac{\not p+m}{p^{2}-m^{2}+i 0} .
$$

The gluon fields have four Lorentz degrees of freedom while a spin-1 massless gluon has only two physical polarizations, which leads to some complications when quantizing the fields. This problem can be solved by adding the so called Faddeev-Popov ghosts $c_{a}$, non-physical massless fields that satisfy the Fermi-Dirac statistics and couple only to gluons, whose lagrangian is ${ }^{30}$

$$
\mathscr{L}_{\text {ghost }}=-\partial^{\mu} \bar{c}_{a} \partial_{\mu} c_{a}+g_{s} f^{a b c} \partial_{\mu} \bar{c}_{a} c_{b} G_{c}^{\mu}
$$

The introduction of these fields allows the quantization of the gluon fields, generating the following propagator for gluons ${ }^{29}$

$$
i \delta_{a b} D^{\mu \nu}(x-y)=\left\langle 0\left|T\left[G_{a}^{\mu}(x) G_{b}^{\nu}(y)\right]\right| 0\right\rangle=i \delta_{a b} \int \frac{\mathrm{d}^{4} k}{(2 \pi)^{4}} \frac{\mathrm{e}^{-i k(x-y)}}{k^{2}+i 0}\left[-g^{\mu \nu}+(1-\epsilon) \frac{k^{\mu} k^{\nu}}{k^{2}+i 0}\right] .
$$

In momentum space, the gluon propagator is given by

$$
i \delta_{a b} D^{\mu \nu}(k)=i \delta_{a b}\left[-g^{\mu \nu}+(1-\epsilon) \frac{k^{\mu} k^{\nu}}{k^{2}+i 0}\right] .
$$

In perturbative $\mathrm{QCD}^{\dagger}$, the Feynman rules are useful. The gluon and quark propagators ${ }^{\dagger}$ We are presuming that perturbative QCD is valid, which will be discussed in more detail later in Secs. 2.2 and 2.3. 
have already been derived and are shown in Eqs. (2.34) and (2.37). It is necessary now to obtain the rules for the interaction vertices, which are extracted from the interaction term of the QCD lagrangian. There are three possible vertices in QCD:

* the interaction vertex between quarks and gluons given by $i g_{s} \gamma_{\mu} \frac{\lambda^{a}}{2}$ and illustrated in Fig. 5;

* the interaction vertex between three gluons, represented in Fig. 6, and given by $-g_{s} f^{a b c}\left[g_{\mu \nu}(k-q)_{\lambda}+g_{\nu \lambda}(q-p)_{\mu}+g_{\lambda \mu}(p-k)_{\nu}\right]$;

* the interaction vertex between four gluons given by $-i g_{s}^{2}\left[f^{a b e} f^{c d e}\left(g_{\mu \lambda} g_{\nu \rho}-g_{\mu \rho} g_{\nu \lambda}\right)+\right.$ $\left.+f^{a d e} f^{b c e}\left(g_{\mu \nu} g_{\lambda \rho}-g_{\mu \lambda} g_{\nu \rho}\right)+f^{a c e} f^{b d e}\left(g_{\mu \nu} g_{\lambda \rho}-g_{\mu \rho} g_{\nu \lambda}\right)\right]$ and shown in Fig. 7 .

\subsection{QCD Renormalization}

Using the Feynman rules, it is possible to calculate transition amplitudes and write corrections to the propagators and vertices through perturbation theory. However these corrections, which are usually loops, generate divergent integrals. In order to obtain finite results, one needs to regularize and renormalize the theory.

Within the canonical definition of renormalizability, a quantum field theory (QFT) is called renormalizable if all the divergences can be absorbed into a redefinition of a finite number of coupling constants and masses. In other words, the theory should have a finite number of superficially divergent diagrams. Considering that no operator has dimension greater than 4 , it can be shown that the dimensions $d$ of all coupling constants have to satisfy $d \leq 4$, otherwise the number of counterterms to cancel the infinities of the theory will be infinite and the QFT will not be renormalizable. ${ }^{31}$ QCD has only one coupling constant, $g_{s}$, which is dimensionless, thus it is a renormalizable theory. It is important to emphasize that a physical observable in a renormalizable theory should not, in principle, depend on the redefinition employed.

We will exemplify this procedure in QCD studying the one-loop correction to the quark propagator, illustrated in Fig. 8. In momentum space, the perturbative corrections to the quark propagator are

$$
S_{i j}(p)=-i \int \mathrm{d}^{4} x \mathrm{e}^{i p x}\left\langle 0\left|T\left\{q_{i}(x) \bar{q}_{j}(0) \mathrm{e}^{i \int \mathrm{d}^{4} z \mathscr{L}_{\text {int }}(z)}\right\}\right| 0\right\rangle,
$$

where $i$ and $j$ are color indices and $\mathscr{L}_{\text {int }}$ is the interaction part of the QCD Lagrangian, given by the second and third lines of Eq. (2.31). One can rewrite the quark propagator by means of perturbation theory as ${ }^{31}$

$$
S_{i j}(p)=\delta_{i j} S^{(0)}(p)+\delta_{i j} S^{(0)}(p) \Sigma(p) S^{(0)}(p)+\delta_{i j} S^{(0)}(p) \Sigma(p) S^{(0)}(p) \Sigma(p) S^{(0)}(p)+\ldots,
$$

where the function $\Sigma(p)$ is called the quark self-energy and, at order $g_{s}^{2}$, is given by 


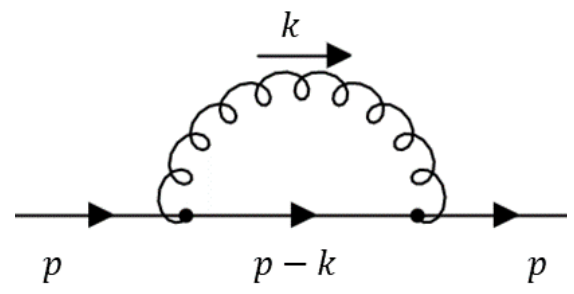

Figure 8 - One-loop correction to the quark propagator.

Source: By the author.

$$
\Sigma^{(1)}(p)=i g_{s}^{2} \frac{4}{3} \int \frac{\mathrm{d}^{4} k}{(2 \pi)^{4}}\left[\gamma^{\mu} S^{(0)}(p-k) \gamma^{\nu}\right] D_{\mu \nu}^{(0)}(k)
$$

Inserting the expressions for the free quark and gluon propagators given respectively in Eqs. (2.34) and (2.37) one finds in the Feynman gauge $(\epsilon=1)$

$$
\Sigma^{(1)}(p)=-i g_{s}^{2} \frac{4}{3} \int \frac{\mathrm{d}^{4} k}{(2 \pi)^{4}} \frac{g_{\mu \nu}\left[\gamma^{\mu}(\not p-\not k+m) \gamma^{\nu}\right]}{k^{2}\left[(p-k)^{2}-m^{2}\right]} .
$$

The integral above is ultraviolet divergent and to deal with it, it is necessary to regularize the integral. There are different methods to perform the regularization and the most used is dimensional regularization ${ }^{32,33}$ because it preserves gauge invariance. This is the mechanism that we will employ in this dissertation.

The main idea is to transform the 4-dimensional integral to a $D$-dimensional one by doing $D=4-2 \epsilon$ and then taking the limit $\epsilon \rightarrow 0$. We also need to introduce the term $\mu^{2 \epsilon} \mu^{-2 \epsilon}$, where $\mu$ is dubbed renormalization scale. The factor $\mu^{-2 \epsilon}$ is responsible for the coupling constant to remain dimensionless as in four dimensions. Using dimensional regularization one gets

$$
\Sigma^{(1)}(p)=-i \mu^{-2 \epsilon} g_{s}^{2} \mu^{2 \epsilon} \frac{4}{3} \int \frac{\mathrm{d}^{D} k}{(2 \pi)^{D}} \frac{g_{\mu \nu}\left[\gamma^{\mu}(\not p-\not k+m) \gamma^{\nu}\right]}{k^{2}\left[(p-k)^{2}-m^{2}\right]} .
$$

In order to solve the integral, one can rewrite the integrand applying Feynamn parametrization that is given by ${ }^{34}$

$$
\frac{1}{A_{1}^{m_{1}} A_{2}^{m_{2}}}=\frac{\Gamma\left(m_{1}+m_{2}\right)}{\Gamma\left(m_{1}\right) \Gamma\left(m_{2}\right)} \int_{0}^{1} \mathrm{~d} x \frac{x^{m_{1}-1}(1-x)^{m_{2}-1}}{\left[A_{1} x+A_{2}(1-x)\right]^{m_{1}+m_{2}}},
$$

with $\Gamma$ being the Euler $\Gamma$ function. So now we have

$$
\Sigma^{(1)}(p)=-i \mu^{-2 \epsilon} g_{s}^{2} \mu^{2 \epsilon} \frac{4}{3} \int \frac{\mathrm{d}^{D} k}{(2 \pi)^{D}} \int_{0}^{1} \mathrm{~d} x \frac{g_{\mu \nu}\left[\gamma^{\mu}(\not p-\not k+m) \gamma^{\nu}\right]}{\left\{k^{2}(1-x)+x\left[(p-k)^{2}-m^{2}\right]\right\}^{2}} .
$$

Shifting the momentum as $l=k-x p$ and performing a Wick rotation, which consists in rotating the time axis $l^{0}$ into an imaginary time direction, one finds 


$$
\Sigma^{(1)}(p)=\mu^{-2 \epsilon} g_{s}^{2} \mu^{2 \epsilon} \frac{4}{3} \int \frac{\mathrm{d}^{D} l}{(2 \pi)^{D}} \int_{0}^{1} \mathrm{~d} x \frac{\gamma_{\nu}[\not p(1-x)+m] \gamma^{\nu}}{\left(l^{2}+\Delta\right)^{2}}
$$

where $\Delta=x m^{2}-x p^{2}(1-x)$ and the linear term in $l$ was disconsidered because it vanishes by symmetry upon integration. One can then see that the quark propagator above can be written in the following manner ${ }^{31}$

$$
\Sigma^{(1)}(p)=\not p \Sigma_{p}^{(1)}(p)+m \Sigma_{m}^{(1)}(p),
$$

with $\Sigma_{p}^{(1)}(p)$ and $\Sigma_{m}^{(1)}(p)$ given by

$$
\begin{aligned}
& \Sigma_{p}^{(1)}(p)=\mu^{-2 \epsilon} g_{s}^{2} \mu^{2 \epsilon} \frac{4}{3} \int \frac{\mathrm{d}^{D} l}{(2 \pi)^{D}} \int_{0}^{1} \mathrm{~d} x \frac{(2-D)(1-x)}{\left(l^{2}+\Delta\right)^{2}}, \\
& \Sigma_{m}^{(1)}(p)=\mu^{-2 \epsilon} g_{s}^{2} \mu^{2 \epsilon} \frac{4}{3} \int \frac{\mathrm{d}^{D} l}{(2 \pi)^{D}} \int_{0}^{1} \mathrm{~d} x \frac{D}{\left(l^{2}+\Delta\right)^{2}} .
\end{aligned}
$$

To solve the integrals above, it is necessary first to compute the integral in $l$ and then expand it for $\epsilon=0$ up to order $\epsilon^{0}$, which generates the expressions

$$
\begin{aligned}
& \Sigma_{p}^{(1)}(p)=\frac{2 \alpha_{s}}{3 \pi} \int_{0}^{1} \mathrm{~d} x(1-x)\left[-\frac{1}{\epsilon}+\gamma_{E}-\ln 4 \pi-\ln \mu^{2}+\ln \Delta+1+\mathscr{O}(\epsilon)\right], \\
& \Sigma_{m}^{(1)}(p)=\frac{4 \alpha_{s}}{3 \pi} \int_{0}^{1} \mathrm{~d} x\left[\frac{1}{\epsilon}-\gamma_{E}+\ln 4 \pi+\ln \mu^{2}-\ln \Delta-\frac{1}{2}+\mathscr{O}(\epsilon)\right],
\end{aligned}
$$

where $\gamma_{E}$ is the Euler constant and $\alpha_{s}=\frac{g_{s}^{2} \mu^{-2 \epsilon}}{4 \pi}$ is known as the QCD coupling and depends on the renormalization scale.

Afterwards, one can calculate the integral in $x$ and finally expand $\Sigma_{p}^{(1)}$ and $\Sigma_{m}^{(1)}$ for small $m^{2}$ up to linear order, since it will not interfere in our purpose of renormalizing the quark propagator up to one-loop. Thus, the results for the functions $\Sigma_{p}^{(1)}$ and $\Sigma_{m}^{(1)}$ are

$$
\begin{aligned}
& \Sigma_{p}^{(1)}(p)=\frac{\alpha_{s}}{3 \pi}\left[-\frac{1}{\epsilon}+\gamma_{E}-\ln 4 \pi+\ln \left(-\frac{p^{2}}{\mu^{2}}\right)-1\right], \\
& \Sigma_{m}^{(1)}(p)=\frac{\alpha_{s}}{3 \pi}\left[\frac{4}{\epsilon}-4 \gamma_{E}+4 \ln 4 \pi-4 \ln \left(-\frac{p^{2}}{\mu^{2}}\right)+6\right] .
\end{aligned}
$$

To obtain the result in 4 dimensions one only needs to take the limit $\epsilon \rightarrow 0$, however the equations above diverge in this limit. Thus it is necessary to renormalize the theory, in other words, one has to redefine the mass and the fields so the divergences will be absorbed by the new parameters. For the one-loop renormalization of the quark propagator only 
the quark field and mass need to be redefined as

$$
q_{i}(x)=Z_{2 F}^{1 / 2} q_{i}^{R}(x) \quad \text { and } \quad m=Z_{m} m^{R} .
$$

The parameters $q_{i}$ and $m$ are called bare quantities, they generate divergent Green functions and are the lagrangian parameters, while $q_{i}^{R}$ and $m^{R}$ are the renormalized ones and lead to finite Green functions. The $Z$-factors are the renormalization constants and are constructed to cancel the divergences order by order, they can be expanded in perturbation theory in the following way

$$
Z_{2 F}=1+Z_{2 F}^{(1)} \frac{\alpha_{s}}{\pi}+\mathscr{O}\left(\alpha_{s}^{2}\right) \quad \text { and } \quad Z_{m}=1+Z_{m}^{(1)} \frac{\alpha_{s}}{\pi}+\mathscr{O}\left(\alpha_{s}^{2}\right) .
$$

Considering the inverse of the quark propagator, we can write in first order in the coupling ${ }^{30}$

$$
S^{-1}(p)=\not p+\not p Z_{2 F}^{(1)} \frac{\alpha_{s}}{\pi}-m^{R}-m^{R}\left(Z_{m}^{(1)}+Z_{2 F}^{(1)}\right) \frac{\alpha_{s}}{\pi}-\not p \Sigma_{p}^{(1)}(p)-m^{R} \Sigma_{m}^{(1)}(p) .
$$

Imposing that the expression above should be finite, we find

$$
Z_{2 F}^{(1)}=-\frac{1}{3 \hat{\epsilon}} \quad \text { and } \quad Z_{m}^{(1)}=-\frac{1}{\hat{\epsilon}},
$$

where we defined $\frac{1}{\hat{\epsilon}}=\frac{1}{\epsilon}-\gamma_{E}+\ln 4 \pi$. It is a choice to take the divergences to be contained in the term $\frac{1}{\hat{\epsilon}}$ and this is denominated modified minimal subtraction scheme or $\overline{\mathrm{MS}}$ scheme, ${ }^{35}$ which is the most popular renormalization scheme. There are also other renormalization schemes as for example the minimal subtraction scheme (MS scheme), where the renormalization constants incorporate just the divergent term $\frac{1}{\epsilon}$.

\subsection{Running Coupling Constant and Running Quark Mass}

As already seen, in the course of renormalization calculations one must choose a renormalization scheme to use. However, if $R\left(q, a_{s}, m\right)$ is a given observable, i.e., a physical quantity, where $q$ is the external momenta, $a_{s}=\frac{\alpha_{s}}{\pi}$ is the QCD coupling and $m$ is the quark mass, then it should not depend on the renormalization scale $\mu$. This requirement gives rise to the following relation

$$
\mu \frac{\mathrm{d}}{\mathrm{d} \mu} R\left(q, a_{s}, m\right)=\left[\mu \frac{\partial}{\partial \mu}+\mu \frac{\mathrm{d} a_{s}}{\mathrm{~d} \mu} \frac{\partial}{\partial a_{s}}+\mu \frac{\mathrm{d} m}{\mathrm{~d} \mu} \frac{\partial}{\partial m}\right] R\left(q, a_{s}, m\right)=0 .
$$

Through this expression, we can define the renormalization group functions ${ }^{\ddagger}$

$$
\begin{aligned}
\beta\left(a_{s}\right) & \equiv-\mu \frac{\mathrm{d} a_{s}}{\mathrm{~d} \mu}=\beta_{1} a_{s}^{2}+\beta_{2} a_{s}^{3}+\ldots & \beta \text { function, } \\
\gamma_{m}\left(a_{s}\right) & \equiv-\frac{\mu}{m} \frac{\mathrm{d} m}{\mathrm{~d} \mu}=\gamma_{m}^{(1)} a_{s}+\gamma_{m}^{(2)} a_{s}^{2}+\ldots & \text { anomalous mass dimension. }
\end{aligned}
$$

${ }_{\ddagger}$ In this work, we define $\gamma$ and $\beta$ functions with the coefficients $\gamma_{1}, \gamma_{2}, \ldots$ and $\beta_{1}, \beta_{2}, \ldots$ as indicated by our Eqs. (2.57) and (2.58). There are several different conventions in the literature involving the signals, factors of $\pi$, among others. In particular, note that our coefficient $\beta_{1}$ is positive. 
The $\gamma$ function rules the quark-mass dependence on the energy while the $\beta$ function rules the coupling constant dependence on the energy given by the renormalization scale $\mu$. Using these functions we can rewrite Eq. (2.56) as

$$
\left[\mu \frac{\partial}{\partial \mu}-\beta\left(a_{s}\right) \frac{\partial}{\partial a_{s}}-\gamma_{m}\left(a_{s}\right) m \frac{\partial}{\partial m}\right] R\left(q, a_{s}, m\right)=0,
$$

which is called the homogeneous renormalization group equation (RGE) of $R\left(q, a_{s}, m\right)$.

Nowadays, both the $\beta$ and $\gamma$ functions are known in perturbation theory up to the fifth-loop coefficients. ${ }^{36,37}$ The difficulty in calculations of this kind is expressed in the interval between the determinations of the coefficients of forth- and fifth-order: $\beta_{4}$ and $\gamma_{4}$ were computed in $1997 ;^{38-40}$ however $\gamma_{5}$ was obtained only in $2014,{ }^{37} 17$ years later, and $\beta_{5}$ in $2017,{ }^{36} 20$ years later.

In our convention, the $\gamma$ function coefficients in the $\overline{\mathrm{MS}}$ scheme with $N_{c}=3$ are $^{41}$

$$
\begin{aligned}
& \gamma_{m}^{(1)}=2, \quad \gamma_{m}^{(2)}=\frac{101}{12}-\frac{5}{18} N_{f}, \quad \gamma_{m}^{(3)}=\frac{1249}{32}-\left(\frac{277}{108}+\frac{5}{3} \zeta_{3}\right) N_{f}-\frac{35}{648} N_{f}^{2}, \\
& \gamma_{m}^{(4)}=\frac{4603055}{20736}+\frac{1060}{27} \zeta_{3}-\frac{275}{4} \zeta_{5}-\left(\frac{91723}{3456}+\frac{2137}{72} \zeta_{3}-\frac{55}{8} \zeta_{4}-\frac{575}{36} \zeta_{5}\right) N_{f}+ \\
& \quad+\left(\frac{2621}{15552}+\frac{25}{36} \zeta_{3}-\frac{5}{12} \zeta_{4}\right) N_{f}^{2}-\left(\frac{83}{7776}-\frac{1}{54} \zeta_{3}\right) N_{f}^{3}, \\
& \gamma_{m}^{(5)}=\frac{99512327}{82944}+\frac{23201233}{62208} \zeta_{3}+\frac{3025}{16} \zeta_{3}^{2}-\frac{349063}{2304} \zeta_{4}-\frac{28969645}{15552} \zeta_{5}+\frac{15125}{32} \zeta_{6}+\frac{25795}{32} \zeta_{7} \\
& +\left(\frac{150736283}{746496}+\frac{391813}{1296} \zeta_{3}+\frac{2365}{144} \zeta_{3}^{2}-\frac{1019371}{6912} \zeta_{4}-\frac{12469045}{31104} \zeta_{5}+\frac{39875}{288} \zeta_{6}+\frac{56875}{432} \zeta_{7}\right) N_{f} \\
& +\left(\frac{91865}{746496}+\frac{803}{2592} \zeta_{3}+\frac{7}{72} \zeta_{4}-\frac{10}{27} \zeta_{5}\right) N_{f}^{3}-\left(\frac{65}{31104}+\frac{5}{1944} \zeta_{3}-\frac{1}{216} \zeta_{4}\right) N_{f}^{4},
\end{aligned}
$$

where $\zeta_{i} \equiv \zeta(i)$ is the Riemann $\zeta$ function and $N_{f}$ the number of active quark flavors.

For the quark mass, Eq. (2.58) can be directly integrated by separation of variables

$$
\int_{m\left(\mu_{1}\right)}^{m\left(\mu_{2}\right)} \frac{\mathrm{d} m}{m}=-\int_{\mu_{1}}^{\mu_{2}} \frac{\mathrm{d} \mu}{\mu} \Longrightarrow \ln \left[\frac{m\left(\mu_{2}\right)}{m\left(\mu_{1}\right)}\right]=\int_{a_{s}\left(\mu_{1}\right)}^{a_{s}\left(\mu_{2}\right)} \mathrm{d} a_{s} \frac{\gamma_{m}\left(a_{s}\right)}{\beta\left(a_{s}\right)} .
$$

So, one obtains

$$
m\left(\mu_{2}\right)=m\left(\mu_{1}\right) \exp \left[\int_{a_{s}\left(\mu_{1}\right)}^{a_{s}\left(\mu_{2}\right)} \mathrm{d} a \frac{\gamma_{m}(a)}{\beta(a)}\right] .
$$

One can see that quark mass ratios are independent of renormalization conventions. The expression above can be rewritten as ${ }^{42}$ 


$$
m(\mu) \equiv \widehat{m}\left[\alpha_{s}(\mu)\right]^{\gamma_{m}^{(1)} / \beta_{1}} \exp \left\{\int_{0}^{a_{s}(\mu)} \mathrm{d} a\left[\frac{\gamma_{m}(a)}{\beta(a)}-\frac{\gamma_{m}^{(1)}}{\beta_{1} a}\right]\right\} .
$$

where $\widehat{m}$ is the renormalization group invariant (RGI) quark mass, which is scale independent. Thus, employing Eq. (2.63) in a perturbative series that is proportional to the quark mass (as the decay width of the Higgs boson into $b$ quarks) results in a series as a function of the invariant mass $\widehat{m}$. This series have the effects of the running quark masses encoded in the $\gamma$ function in the exponential term.

Furthermore, the coefficients of the $\beta$ function up to fifth order are a $^{41}$

$$
\begin{aligned}
\beta_{1} & =\frac{11}{2}-\frac{1}{3} N_{f}, \quad \beta_{2}=\frac{51}{4}-\frac{19}{12} N_{f}, \quad \beta_{3}=\frac{2857}{64}-\frac{5033}{576} N_{f}+\frac{325}{1728} N_{f}^{2} \\
\beta_{4} & =\frac{149753}{768}+\frac{891}{32} \zeta_{3}-\left(\frac{1078361}{20736}+\frac{1627}{864} \zeta_{3}\right) N_{f}+\left(\frac{50065}{20736}+\frac{809}{1296} \zeta_{3}\right) N_{f}^{2}+\frac{1093}{93312} N_{f}^{3}, \\
\beta_{5} & =\frac{8157455}{8192}+\frac{621885}{1024} \zeta_{3}-\frac{88209}{1024} \zeta_{4}-\frac{144045}{256} \zeta_{5}- \\
& -\left(\frac{336460813}{995328}+\frac{1202791}{10368} \zeta_{3}-\frac{33935}{3072} \zeta_{4}-\frac{1358995}{13824} \zeta_{5}\right) N_{f} \\
& +\left(\frac{25960913}{995328}+\frac{698531}{41472} \zeta_{3}-\frac{5263}{2304} \zeta_{4}-\frac{5965}{648} \zeta_{5}\right) N_{f}^{2}- \\
& -\left(\frac{630559}{2985984}+\frac{24361}{62208} \zeta_{3}-\frac{809}{6912} \zeta_{4}-\frac{115}{1152} \zeta_{5}\right) N_{f}^{3}+\left(\frac{1205}{1492992}-\frac{19}{5184} \zeta_{3}\right) N_{f}^{4} . \quad(2.64)
\end{aligned}
$$

As we did for the quark mass, Eq. (2.57) can be integrated by separation of variables

$$
\int_{a_{s}\left(\mu_{1}\right)}^{a_{s}\left(\mu_{2}\right)} \frac{\mathrm{d} a_{s}}{\beta\left(a_{s}\right)}=-\int_{\mu_{1}}^{\mu_{2}} \frac{\mathrm{d} \mu}{\mu}=\ln \frac{\mu_{1}}{\mu_{2}} .
$$

At one-loop this equation can be solved analytically

$$
\int_{a_{s}\left(\mu_{1}\right)}^{a_{s}\left(\mu_{2}\right)} \frac{\mathrm{d} a_{s}}{\beta_{1} a_{s}^{2}}=\frac{1}{\beta_{1}}\left[\frac{1}{a_{s}\left(\mu_{1}\right)}-\frac{1}{a_{s}\left(\mu_{2}\right)}\right]=\ln \frac{\mu_{1}}{\mu_{2}} \Longrightarrow a_{s}\left(\mu_{2}\right)=\frac{a_{s}\left(\mu_{1}\right)}{1-a_{s}\left(\mu_{1}\right) \beta_{1} \ln \frac{\mu_{1}}{\mu_{2}}} .
$$

Since $\beta_{1}$ is positive (in our convention), $a(\mu)$ decreases logarithmically for $\mu_{2}>\mu_{1}$ and vanishes when $\mu_{2} \rightarrow \infty$. This is the asymptotic freedom property of QCD: at high energies (small distances) the interaction between quark and antiquark becomes weaker; we can neglect this interaction when the pair is close enough, so they behave as free particles.

For the other terms of the $\beta$ function, the integral at Eq. (2.65) can be done numerically and the running of $\alpha_{s}$ can be seen in Fig. 9. The black line is the theoretical prediction using the known coefficients of the $\beta$ function. ${ }^{43}$ One can see that for high energies the 


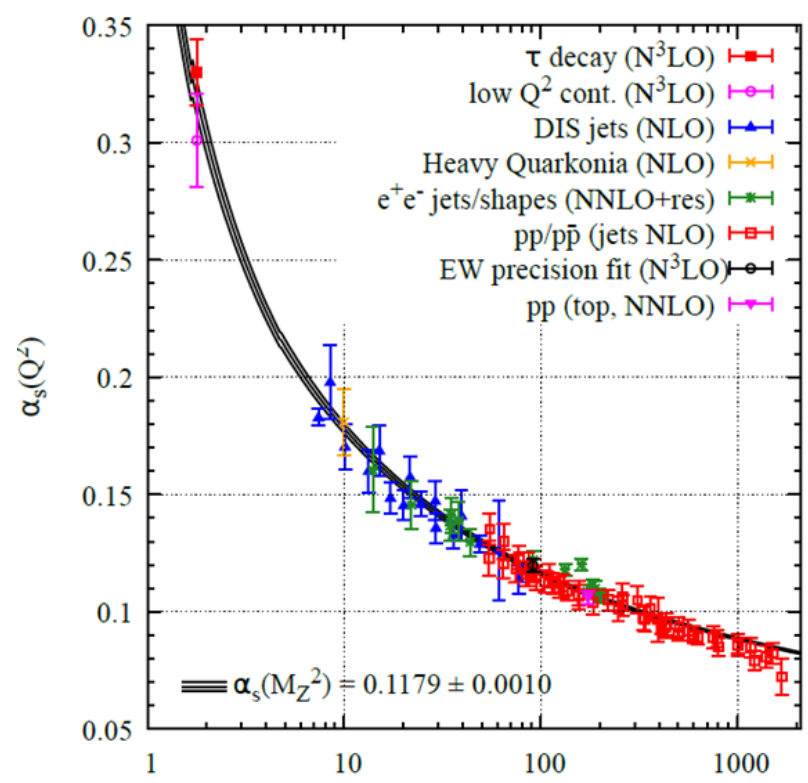

Figure 9 - Experimental determination of $\alpha_{s}$ collected by Particle Data Group.

Source: ZYLA et $a .^{43}$

coupling is relatively small, it is possible then to use perturbation theory in this regime to study QCD. The colored dots in Fig. 9 are the extractions done in different energies, where the terms in the brackets (NLO, NNLO and $\mathrm{N}^{3} \mathrm{LO}$ ) are the order of perturbation theory used in the extraction of $\alpha_{s} .{ }^{43}$ It is possible to notice how effectively the extractions of the coupling fit the theoretical prediction for distinct energies. This plot (or a similar one for the quark mass) can only be done if all the extractions of the coupling from experimental data are given in the same renormalization scheme, which in this case is the $\overline{\mathrm{MS}}$. This occurs because the QCD coupling $\alpha_{s}$ and the quark mass $m$ are not physical parameters due to their dependence on the renormalization scheme and scale.

The running of $a_{s}$ can be characterized by a starting value $a_{s}\left(\mu_{1}\right)$ or the point $\Lambda$ where the coupling diverges, known as Landau pole. In the case of Eq. (2.66), where just the first coefficient of the $\beta$ function was used, the Landau pole is where the denominator vanishes and is given by

$$
\Lambda=\mu_{1} \mathrm{e}^{\frac{-1}{\beta^{a_{s}\left(\mu_{1}\right)}}} .
$$

The constant $\Lambda$ is independent of scale, thus it is a RGI quantity (as $\widehat{m}$ ) and sets an energy scale for non-perturbative QCD. Through this constant it is possible to define, for a particular scheme, the point around which the coupling becomes excessively high to use perturbation theory. The Landau pole is scheme dependent hence it is not a physical quantity. However, it can be written in a scale independent form to all orders as

$$
\Lambda=\mu \mathrm{e}^{-\frac{1}{\beta_{1} \mu}}\left[a_{s}(\mu)\right]^{-\frac{\beta_{2}}{\beta_{1}^{2}}} \exp \left[\int_{0}^{a_{s}(\mu)} \frac{\mathrm{d} a}{\tilde{\beta}(a)}\right],
$$


where

$$
\frac{1}{\tilde{\beta}(a)} \equiv \frac{1}{\beta(a)}-\frac{1}{\beta_{1} a^{2}}+\frac{\beta_{2}}{\beta_{1}^{2} a} .
$$

As we already said, the coupling constant depends on the renormalization scheme, thus it is possible to write a perturbative scheme transformation from a coupling $a_{s}$ to a new coupling $a_{s}^{\prime}$ in a different scheme in the general form

$$
a_{s}^{\prime}=a_{s}+c_{1} a_{s}^{2}+c_{2} a_{s}^{3}+c_{3} a_{s}^{4}+\ldots .
$$

A remarkable result due to Celmaster and Gonsalves is that the new parameter $\Lambda^{\prime}$ in this new scheme only depends on $c_{1}$ through the relation below ${ }^{44}$

$$
\Lambda^{\prime}=\Lambda \mathrm{e}^{c_{1} / \beta_{1}} .
$$

Since the parameters $\Lambda$ and $\Lambda^{\prime}$ are related by only one constant, one can rewrite the transformation of $a_{s}$ to $a_{s}^{\prime}$ as a function of this variable. At one loop, this is an old idea but it has been extended to all orders recently by Boito, Jamin and Miravitllas, ${ }^{21}$ and, for $C=-\frac{2 c_{1}}{\beta_{1}}$, this relation is given by

$$
\frac{1}{a_{s}^{\prime}}+\frac{\beta_{2}}{\beta_{1}} \ln a_{s}^{\prime} \equiv \beta_{1}\left(\ln \frac{\mu}{\Lambda}+\frac{C}{2}\right)=\frac{1}{a_{s}}+\frac{\beta_{1}}{2} C+\frac{\beta_{2}}{\beta_{1}} \ln a_{s}-\beta_{1} \int_{0}^{a_{s}} \frac{\mathrm{d} a}{\tilde{\beta}(a)} .
$$

This equation can be solved iteratively, generating then the perturbative relation between the new coupling $a_{s}^{\prime}$ and the old one $a_{s}$. For $N_{f}=5$ we have

$$
\begin{aligned}
& a_{s}^{\prime}=a_{s}-\frac{23}{12} C a_{s}^{2}+\left(\frac{17521}{152352}-\frac{29}{12} C+\frac{529}{144} C^{2}\right) a_{s}^{3}+\left(\frac{1075144295}{756884736}-\frac{138625}{39744} C+\frac{3335}{288} C^{2}\right. \\
& \left.-\frac{12167}{1728} C^{3}-\frac{11027}{2484} \zeta_{3}\right) a_{s}^{4}+\left[\frac{1743062365679}{278533582848}-\left(\frac{1998367067}{197448192}-\frac{11027}{648} \zeta_{3}\right) C+\frac{380833}{13824} C^{2}\right. \\
& \left.-\frac{199433}{5184} C^{3}+\frac{279841}{20736} C^{4}+\frac{2149885883}{65816064} \zeta_{3}+\frac{11027}{1728} \zeta_{4}+\frac{11976865}{476928} \zeta_{5}\right] a_{s}^{5}+\ldots,
\end{aligned}
$$

and reversing the equation above one gets

$$
\begin{aligned}
& a_{s}=a_{s}^{\prime}+\frac{23}{12} C a_{s}^{\prime 2}-\left(\frac{17521}{152352}-\frac{29}{12} C-\frac{529}{144} C^{2}\right) a_{s}^{\prime 3}-\left(\frac{1075144295}{756884736}-\frac{63215}{26496} C-\frac{3335}{288} C^{2}\right. \\
& \left.-\frac{12167}{1728} C^{3}-\frac{11027}{2484} \zeta_{3}\right) a_{s}^{\prime 4}-\left[\frac{1732010889803}{278533582848}+\left(\frac{778160225}{98724096}-\frac{11027}{324} \zeta_{3}\right) C-\frac{164135}{6912} C^{2}\right. \\
& \left.-\frac{199433}{5184} C^{3}-\frac{279841}{20736} C^{4}-\frac{2149885883}{65816064} \zeta_{3}+\frac{11027}{1728} \zeta_{4}+\frac{11976865}{476928} \zeta_{5}\right] a_{s}^{\prime 5}+\ldots
\end{aligned}
$$

The formulas above generate the coupling in a new renormalization scheme, known as the $C$-scheme. ${ }^{21}$ Modifying the value of $C$, which alters the renormalization scheme, 
can generate a more perturbative series, in other words, a smaller value of $a_{s}$; or a less perturbative series, with a higher value for the coupling. We will show in Ch. 5 how a renormalization scheme variation can influence a perturbative series, as well as its convergence properties. 


\section{QCD IN THE DECAY OF THE HIGGS INTO BOTTOM QUARKS}

Since the Higgs boson interacts with all massive particles of the SM, it can decay in many different channels. One possibility is the decay into a fermion-antifermion pair. The Higgs boson is more likely to decay to heavier fermions since, as seen before, the coupling strength of their interaction is proportional to the fermion mass. The most common decay for the observable Higgs, whose mass is $125.10 \pm 0.14 \mathrm{GeV},{ }^{43}$ is into a bottom-antibottom pair as we can see in Fig. 10, that illustrates the branching ratios of the Higgs decay modes as a function of the Higgs mass. This decay occurs approximately $60 \%$ of the time and is the dominant mode because the Higgs boson does not have enough mass to decay into the heaviest fermion, the top quark.

Another possible channel is the decay into a pair of massive gauge bosons. The channel $H \rightarrow W W$ (with one of the $W$ off shell) is the second-to most likely to occur, as indicated in Fig. 10, and represents approximately $22 \%$ of the Higgs decays. The Higgs boson can also decay into massless gauge bosons, the photon and the gluon, through an intermediate loop of virtual heavy quarks because the Higgs boson does not couple with massless particles in the SM. Among these two channels, the most probable one is the decay into a pair of gluons, occurring around $9 \%$ of the time.

In this section we introduce the framework for the calculation of the decay rate of the Higgs boson into bottom quarks. First we will calculate the leading order term and then the method of the master integrals will be introduced in order to calculate the next-toleading-order term of this decay width. After that, we will discuss the state-of-the-art for this observable and all presently known terms due to higher-order QCD corrections.

\subsection{Leading-Order Term}

We will start with the calculation of the decay rate of the Higgs decay into bottom quarks at leading order (LO) in perturbative QCD. As already seen, the lagrangian that describes the interaction between the Higgs and quarks is

$$
\mathscr{L}_{\mathrm{int}}=-\frac{y_{q}}{\sqrt{2}} H \bar{q}_{f}^{\alpha} q_{f}^{\alpha},
$$

where $q_{f}^{\alpha}$ represents the quark field of color $\alpha$ and $y_{q}=\frac{\sqrt{2} m_{q}}{v}$. Thus, the amplitude $\mathscr{M}$ for the decay $H\left(p_{1}\right) \rightarrow b\left(p_{2}\right) \bar{b}\left(p_{3}\right)$ at lowest order is

$$
\mathscr{M}=\frac{N_{c} m_{b}^{2}}{v^{2}} \operatorname{Tr}\left[\left(\not p_{2}-m_{b}\right)\left(\not p_{3}-m_{b}\right)\right]
$$

where $p_{2}$ and $p_{3}$ are the four-momenta of $b$ and $\bar{b}$ respectively and $N_{c}$ represents the number of colors. Therefore, in the Higgs rest frame one finds 


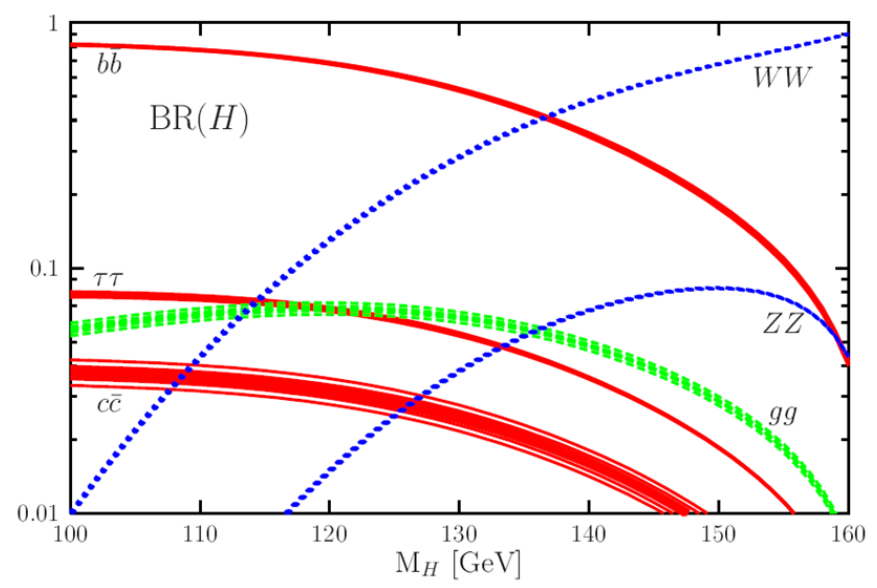

Figure 10 - Branching ratios of different decay modes of the SM Higgs as a function of its mass including the uncertainties in 2005 of the quark masses and the QCD coupling.

Source: DJOUADI. ${ }^{28}$

$$
|\mathscr{M}|^{2}=\frac{2 N_{c} m_{b}^{2}}{v^{2}}\left(m_{H}^{2}-4 m_{b}^{2}\right),
$$

where $m_{H}$ is the Higgs mass. With this result and the phase-space factor for the 2-body decays $^{29}$ we can calculate the decay width $\Gamma(H \rightarrow b \bar{b})$ at lowest order, which reads

$$
\Gamma(H \rightarrow b \bar{b})=\frac{N_{c} m_{b}^{2}}{8 \pi v^{2}} m_{H}\left(1-\frac{4 m_{b}^{2}}{m_{H}^{2}}\right)^{3 / 2} .
$$

Although it is possible to calculate the corrections to this result using this formalism, at higher orders, diagrams with real gluon emissions appear, which are, in practice, 3-body (or more) decays. These diagrams must be included since in the final state we will, eventually, have hadronic jets. For this reason and also to avoid issues related to infrared divergences in the emission of real gluons, it is advantageous to re-express this result using the Optical Theorem in terms of the imaginary part of the scalar-current $b \bar{b}$ correlator.

We will now recall the Optical Theorem, which is a straightforward consequence of the $S$-matrix unitarity. Writing it in the following way: $S=1-i T$, where $T$ is the transition matrix, we have $i\left(T-T^{\dagger}\right)=T^{\dagger} T$. The matrix element of this equation can be calculated between the initial state $|i\rangle$ and the final one $|f\rangle$ as

$$
i\left\langle f\left|T-T^{\dagger}\right| i\right\rangle=\widetilde{\sum_{n}}\left\langle f\left|T^{\dagger}\right| n\right\rangle\langle n|T| i\rangle,
$$

where the right-hand side was calculated introducing a complete set of intermediate states $|n\rangle$ and the notation below was used

$$
\widetilde{\sum_{n}} \equiv \sum_{n} \frac{1}{(2 n)^{3 n_{j}}} \int \prod_{l=1}^{n_{j}} \frac{\mathrm{d}^{3} p_{l}}{E_{l}},
$$

with $n_{j}$ representing the number of particles in the state $|n\rangle$. The $T$-matrix elements 
can be expressed as a product of invariant $\mathscr{M}$-matrix elements and the 4 -momentum conservation delta function. Thus, Eq. (3.3) can be written as

$$
-i\left[\mathscr{M}_{i \rightarrow f}-\mathscr{M}_{f \rightarrow i}^{*}\right]=\widetilde{\sum_{n}} \mathscr{M}_{f \rightarrow n}^{*} \mathscr{M}_{i \rightarrow n}(2 \pi)^{4} \delta^{4}\left(p_{i}-p_{n}\right),
$$

times an overall delta function $(2 \pi)^{4} \delta^{4}\left(p_{i}-p_{f}\right)$. The above identity can be written in a more compact way which corresponds to the standard form of the Optical Theorem

$$
2 \operatorname{Im}\left(\mathscr{M}_{i \rightarrow i}\right)=\widetilde{\sum_{n}}(2 \pi)^{4} \delta^{4}\left(p_{i}-p_{n}\right)\left|\mathscr{M}_{i \rightarrow n}\right|^{2} .
$$

If in the initial state there is just one particle, we are dealing with self-energy diagrams and the theorem can be rewritten as

$$
\operatorname{Im}\left(\mathscr{M}_{i \rightarrow i}\right)=m \Gamma(i \rightarrow \text { all }),
$$

where $\Gamma(i \rightarrow$ all $)$ is the decay width of the initial particle and $m$ is its mass. Hence, it is possible to obtain the decay width from the imaginary part of a correlator, which is the strategy used at high orders, because the calculations are simpler.

We can now calculate the decay width of the Higgs into bottom quarks in its dominant term using the Optical Theorem. The decay width that we want to obtain is related to the correlator of two scalar currents, which is defined by the expression below

$$
\Pi\left(p^{2}\right) \equiv i \int \mathrm{d} x \mathrm{e}^{i p x}\left\langle\Omega\left|T\left\{j(x) j^{\dagger}(0)\right\}\right| \Omega\right\rangle,
$$

with $|\Omega\rangle$ representing the non-perturbative QCD vacuum. The scalar current $j(x)$ arises from the interaction between the Higgs and the bottom quarks and is given by

$$
j(x)=m_{q}: \bar{q}_{f}(x) q_{f}(x):,
$$

where $: \bar{q}_{f}(x) q_{f}(x)$ : are the normal-ordered fields. This current is a RGI, as the invariant quark mass $\widehat{m}$, defined in Eq. (2.63).

In higher-order calculations, it is common to first compute the correlator $\Pi\left(p^{2}\right)$ and later take the imaginary part, that originates in the logarithms. We will use this method to calculate the dominant term of the decay rate of the Higgs into $b$ quarks. Performing the contractions, the leading-order term of Eq. (3.7) can be written as

$$
\Pi_{1}\left(p^{2}\right)=-i N_{c} m_{b}^{2} \int \mathrm{d} x \mathrm{e}^{i p x} \operatorname{Tr}[S(x) S(-x)],
$$

where $S(x)$ is the free quark propagator. The diagram of the expression above is illustrated in Fig. 11, which shows the Higgs self-energy with a loop of bottom quarks. Substituting the expressions for the quark propagators leads to the result below

$$
i \Pi_{1}\left(p^{2}\right)=-N_{c} m_{b}^{2} \int \frac{\mathrm{d}^{4} k}{(2 \pi)^{4}} \frac{\operatorname{Tr}\left[\left(\not k+\not p+m_{b}\right)\left(\not k+m_{b}\right)\right]}{\left[(k+p)^{2}-m_{b}^{2}\right]\left(k^{2}-m_{b}^{2}\right)} .
$$

In order to solve the integral above, a Feynman parameter will be introduced, as explained in Eq. (2.42). Defining the shifted momentum as $l=k+x p$, Eq. (3.10) can be rewritten, 


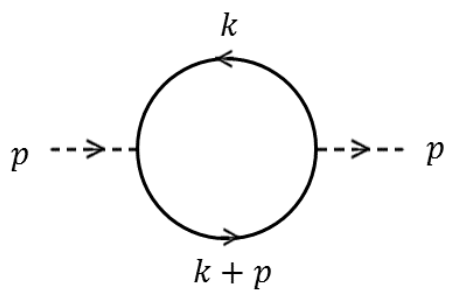

Figure 11 - One-loop diagram of bottom quarks.

Source: By the author.

dropping the linear terms in $l$, which vanish by symmetry upon integration, as

$$
i \Pi_{1}\left(p^{2}\right)=-4 N_{c} m_{b}^{2} \int_{0}^{1} \mathrm{~d} x \int \frac{\mathrm{d}^{4} l}{(2 \pi)^{4}} \frac{l^{2}+\Delta}{\left(l^{2}-\Delta\right)^{2}},
$$

where $\Delta=m_{b}^{2}-(1-x) x p^{2}$. In dimensional regularization, after doing a Wick rotation, calculating the integral in $l$ and taking the limit $\epsilon \rightarrow 0$, as we did in Sec. 2.2, the result is

$$
\Pi_{1}\left(p^{2}\right)=4 N_{c} m_{b}^{2} \int_{0}^{1} \mathrm{~d} x \frac{3 \Delta}{16 \pi^{2}}\left[-\frac{1}{\epsilon}-\frac{1}{3}+\gamma_{E}-\ln 4 \pi+\ln \Delta\right]+\mathscr{O}(\epsilon) .
$$

Calculating the integral $\int_{0}^{1} \mathrm{~d} x \Delta$ we get the following expression

$$
\Pi_{1}\left(p^{2}\right)=N_{c} \frac{3 m_{b}^{2}}{4 \pi^{2}}\left[\left(-\frac{1}{\epsilon}+\gamma_{E}-\ln 4 \pi-\frac{1}{3}\right)\left(m_{b}^{2}-\frac{p^{2}}{6}\right)+\int_{0}^{1} \mathrm{~d} x \Delta \ln \Delta\right] .
$$

To obtain the decay rate it is necessary to calculate the imaginary part of the scalar correlator $\Pi_{1}$. One can note that only the last term of the above expression will contribute to the imaginary part, which comes from the logarithm when $\Delta<0$, because all the other ones are real. Since the divergent part is purely real, the decay rate can be computed without necessarily renormalizing the result.

The values of $x$ that contribute to the imaginary part are between $x_{1}$ and $x_{2}$, the roots of the equation $\Delta=0$, due to the fact that $\Delta<0$ in this interval. Reminding that $\operatorname{Im}[\ln (-\alpha \pm i \epsilon)]= \pm \pi$, one finds

$$
\operatorname{Im} \Pi_{1}\left(p^{2}\right)=N_{c} \frac{3 m_{b}^{2}}{4 \pi^{2}}(-\pi) \int_{x_{1}}^{x_{2}} \mathrm{~d} x \Delta .
$$

Solving the last integral we finally get in the Higgs rest frame

$$
\operatorname{Im} \Pi_{1}\left(p^{2}\right)=N_{c} \frac{m_{b}^{2}}{8 \pi} m_{H}^{2}\left(1-\frac{4 m_{b}^{2}}{m_{H}^{2}}\right)^{3 / 2} .
$$

Comparing this result with the decay width of Eq. (3.2) the relation between $\operatorname{Im} \Pi_{1}$ and $\Gamma(H \rightarrow b \bar{b})$ is given by 


$$
\Gamma(H \rightarrow b \bar{b})=\frac{1}{v^{2} m_{H}} \operatorname{Im} \Pi_{1}\left(p^{2}\right),
$$

which is predicted by the Optical Theorem. The factor of $v^{2}$ has to be added in the expression predicted by the Optical Theorem in Eq. (3.6) because the current we are dealing with, defined in Eq. (3.8), does not contain the vacuum expectation value of Higgs, which appears in the Feynman rules for the vertex, as seen in Sec. 2.1.2, used in the calculations of $\Gamma(H \rightarrow b \bar{b})$ at leading order.

Using Eq. (3.16) it is possible to determine the corrections to the decay rate $\Gamma(H \rightarrow b \bar{b})$ through the calculations at higher orders of the imaginary part of the scalar correlator. We will calculate the next-to-leading-order term in QCD of the decay width of the Higgs into $b$ quarks. However, it is necessary previously to introduce the method of master integrals to calculate the scalar correlator $\Pi$ up to this order.

\subsection{Master Integrals Method}

\subsubsection{One-Loop Massless Propagator}

We start by introducing the master integral method for the one-loop massless case. We will work in the massless limit because the b-quark mass, which is around $4.18 \mathrm{GeV}$, is much smaller than the Higgs mass, which is $125.1 \mathrm{GeV}$, thus the squared ratio of these two masses is of the order of $\mathscr{O}\left(10^{-3}\right)$. Although some precision is lost using this limit, the calculations are much simpler. In addition, the results for the imaginary part of the scalar correlator up to $\alpha_{s}^{4}$ are known only in the limit $m_{b}^{2} \ll m_{H}^{2}$.

Consider the general one-loop massless propagator diagram shown in Fig. 12. The Lorentz scalar master integral for this diagram can be written in the following manner ${ }^{45}$

$$
\int \frac{\mathrm{d}^{D} k}{d_{1}^{n_{1}} d_{2}^{n_{2}}}=i \pi^{D / 2} \widetilde{G}_{1}\left(n_{1}, n_{2}\right)
$$

where the denominators $d_{1}$ and $d_{2}$ are defined as $d_{1}=-(k+p)^{2}$ e $d_{2}=-k^{2}$. This integral is a generalization of the previous case, for any $n_{1}$ and $n_{2}$, and is symmetric under the substitution $1 \leftrightarrow 2$.

Our main aim is to compute the function $\widetilde{G}_{1}$ for $p^{2}<0$, which is below the threshold of production of a real pair of particles. It will be important for the next calculations, serving as a master integral.

To determine it, a process similar to that employed in Sec. 3.1 will be used. First, a Wick rotation will be done and instead of using Feynman parametrization, we will employ the $\alpha$ parametrization (also known as Schwinger parametrization), which for a general integral is given by ${ }^{46}$

$$
\frac{1}{A_{1}^{m_{1}} A_{2}^{m_{2}} \cdots A_{n}^{m_{n}}}=\frac{1}{\Gamma\left(m_{1}\right) \cdots \Gamma\left(m_{n}\right)} \int \mathrm{d} \alpha_{1} \cdots \mathrm{d} \alpha_{n} \exp \left(\sum_{i}-\alpha_{i} A_{i}\right) \alpha_{1}^{m_{1}-1} \cdots \alpha_{n}^{m_{n}-1} .
$$




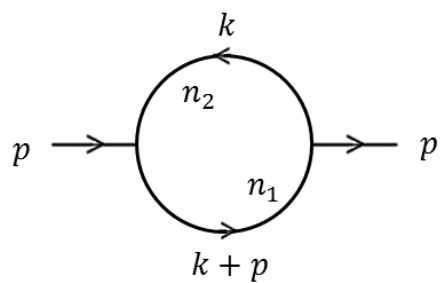

Figure 12 - One-loop massless propagator diagram, where $n_{i}$ is the power of the propagator.

Source: By the author.

The integrals in $\alpha_{i}$ are calculated between 0 and infinity. In this way the function $\widetilde{G}_{1}\left(n_{1}, n_{2}\right)$ can be written as

$$
\widetilde{G}_{1}\left(n_{1}, n_{2}\right)=\frac{\pi^{-D / 2}}{\Gamma\left(n_{1}\right) \Gamma\left(n_{2}\right)} \int \mathrm{d}^{D} k_{E} \mathrm{~d} \alpha_{1} \mathrm{~d} \alpha_{2} \quad \mathrm{e}^{-\alpha_{1}\left(k_{E}+p_{E}\right)^{2}-\alpha_{2} k_{E}^{2}} \quad \alpha_{1}^{n_{1}-1} \alpha_{2}^{n_{2}-1} .
$$

With the following change of variable $Q_{E}=k_{E}+\frac{\alpha_{1}}{\alpha_{1}+\alpha_{2}} p_{E}$ and solving the integral of $Q_{E}$

$$
\widetilde{G}_{1}\left(n_{1}, n_{2}\right)=\frac{1}{\Gamma\left(n_{1}\right) \Gamma\left(n_{2}\right)} \int \mathrm{d} \alpha_{1} \mathrm{~d} \alpha_{2} \exp \left[-\frac{\alpha_{1} \alpha_{2}}{\alpha_{1}+\alpha_{2}} p_{E}^{2}\right] \frac{\alpha_{1}^{n_{1}-1} \alpha_{2}^{n_{2}-1}}{\left(\alpha_{1}+\alpha_{2}\right)^{D / 2}}
$$

Another change of variables is needed to solve the remaining integrals: $\alpha_{1}=\eta x$ and $\alpha_{2}=\eta(1-x)$, giving the result below

$$
\widetilde{G}_{1}\left(n_{1}, n_{2}\right)=\left(p_{E}^{2}\right)^{D / 2-n_{1}-n-2} \frac{\Gamma\left(-D / 2+n_{1}+n_{2}\right)}{\Gamma\left(n_{1}\right) \Gamma\left(n_{2}\right)} \int_{0}^{1} \mathrm{~d} x x^{D / 2-n_{2}-1}(1-x)^{D / 2-n_{1}-1} .
$$

The integral above generates a Euler $B$ function and so the final result is

$$
\widetilde{G}_{1}\left(n_{1}, n_{2}\right)=\left(p_{E}^{2}\right)^{D / 2-n_{1}-n-2} \frac{\Gamma\left(-D / 2+n_{1}+n_{2}\right) \Gamma\left(D / 2-n_{1}\right) \Gamma\left(D / 2-n_{2}\right)}{\Gamma\left(n_{1}\right) \Gamma\left(n_{2}\right)} .
$$

Eq. (3.17) can be written in terms of a new function $G_{1}\left(n_{1}, n_{2}\right)$ as

$$
\int \frac{\mathrm{d}^{D} k}{d_{1}^{n_{1}} d_{2}^{n_{2}}}=i \pi^{D / 2}\left(-p^{2}\right)^{D / 2-n_{1}-n_{2}} G_{1}\left(n_{1}, n_{2}\right)
$$

where $G_{1}\left(n_{1}, n_{2}\right)$ is dimensionless and the integral dimension is all contained in the factor of $p^{2}$. Finally, the function $G_{1}\left(n_{1}, n_{2}\right)$ is given by

$$
G_{1}\left(n_{1}, n_{2}\right)=\frac{\Gamma\left(-D / 2+n_{1}+n_{2}\right) \Gamma\left(D / 2-n_{1}\right) \Gamma\left(D / 2-n_{2}\right)}{\Gamma\left(n_{1}\right) \Gamma\left(n_{2}\right)} .
$$

From the definition in Eq. (3.21), the following expression, that will be helpful later, can be computed

$$
\frac{G_{1}\left(n_{1}, n_{2}+1\right)}{G_{1}\left(n_{1}, n_{2}\right)}=-\frac{\left(D-2 n_{1}-2 n_{2}\right)\left(D-n_{1}-n_{2}-1\right)}{n_{2}\left(D-2 n_{2}-2\right)} .
$$

It is possible now to calculate the leading-order term for the decay rate in the limit 
where the $b$-quark mass is much smaller than the Higgs mass using this technique. As we already discussed, this limit is a good approximation since the squared ratio of these two masses is of the order of $\mathscr{O}\left(10^{-3}\right)$. Then, rewritten Eq. (3.10) in $D$ dimensions when $m_{b}^{2} \ll m_{H}^{2}$ gives us

$$
i \Pi_{1}=-4 N_{c} m_{b}^{2} \mu^{2 \epsilon} \mu^{-2 \epsilon} \int \frac{\mathrm{d}^{D} k}{(2 \pi)^{D}} \frac{\operatorname{Tr}[(\not k+\not p) \not k]}{(k+p)^{2} k^{2}} .
$$

Computing the trace in $D$ dimensions and rewriting it in terms of the variables $d_{1}$ and $d_{2}$ defined before the result is expressed in terms of scalar integrals as

$$
\Pi_{1}=-2 i N_{c} m_{b}^{2} \mu^{2 \epsilon} \mu^{-2 \epsilon} \int \frac{\mathrm{d}^{D} k}{(2 \pi)^{D}} \frac{d_{1}+d_{2}+p^{2}}{d_{1} d_{2}} .
$$

The integrals will be solved using the one-loop master integral given in Eq. (3.20). Since it depends on the function $G_{1}\left(n_{1}, n_{2}\right)$, which is equal to zero if $n_{1}=0$ or $n_{2}=0$, only the last term of the integral will contribute. In this way

$$
\Pi_{1}=-2 N_{c} m_{b}^{2} \mu^{2 \epsilon} \mu^{-2 \epsilon} \frac{(-p)^{D / 2-1}}{(4 \pi)^{D / 2}} g_{1},
$$

where the notation $g_{1}=G_{1}(1,1)$ was introduced. Reminding that $D=4-2 \epsilon$, it is possible to expand $\Pi_{1}$ near $\epsilon=0$ reaching the result below

$$
\Pi_{1}=\frac{N_{c} m_{b}^{2} p^{2}}{8 \pi^{2}}\left[\frac{1}{\epsilon}+2-\gamma_{E}+\ln 4 \pi-\ln \left(-\frac{p^{2}}{\mu^{2}}\right)\right]+\mathscr{O}(\epsilon) .
$$

One can calculate the imaginary part of this expression in order to obtain the decay rate $\Gamma(H \rightarrow b \bar{b})$. Since only the logarithm has an imaginary part, it is not necessary to renormalize the result. Hence, taking the imaginary part one finds

$$
\operatorname{Im} \Pi_{1}=\frac{N_{c} m_{b}^{2} p^{2}}{8 \pi}
$$

which, when computed in the Higgs rest frame (where $p^{2}=m_{H}^{2}$ ), coincides exactly with the result of Eq. (3.15) in the limit $m_{b}^{2} \ll m_{H}^{2}$. It is possible to see how powerful and simple this technique is compared to the evaluation from scratch using Feynman rules.

\subsubsection{Two-Loop Massless Propagator}

We now generalize the master integral method for the two-loop massless case, which will be used to compute the first-order correction to the decay rate $\Gamma(H \rightarrow b \bar{b})$. Consider the general two-loop massless propagator diagram shown in Fig. 13. For this diagram, the following scalar integral can be written ${ }^{45}$

$$
\int \frac{\mathrm{d}^{D} k_{1} \mathrm{~d}^{D} k_{2}}{d_{1}^{n_{1}} d_{2}^{n_{2}} d_{3}^{n_{3}} d_{4}^{n_{4}} d_{5}^{n_{5}}}=i \pi^{D / 2}\left(-p^{2}\right)^{D-\sum_{i} n_{i}} G_{2}\left(n_{1}, n_{2}, n_{3}, n_{4}, n_{5}\right),
$$

where the power of $-p^{2}$ is determined by the equation dimensionality and the denominators 


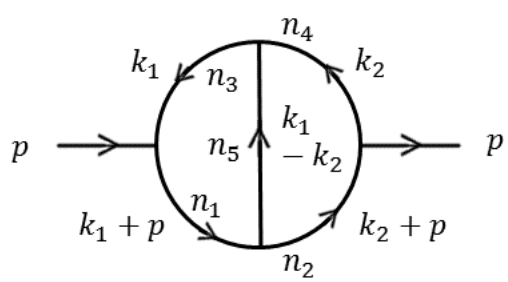

Figure 13 - Two-loop massless propagator diagram, where $n_{i}$ is the power of the propagator.

Source: By the author.

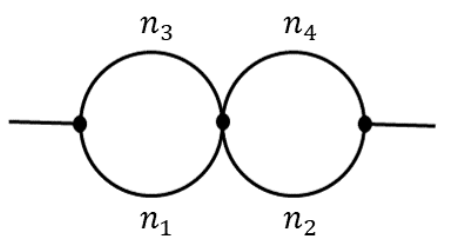

Figure $14-$ Diagram for the case $n_{5}=0$.

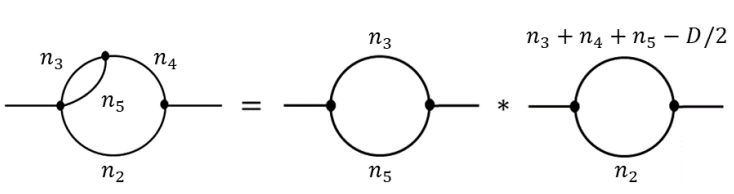

Figure 15 - Diagram for the case $n_{1}=0$.

Source: By the author.

$d_{i}$ are defined as

$$
d_{1}=-\left(k_{1}+p\right)^{2}, \quad d_{2}=-\left(k_{2}+p\right)^{2}, \quad d_{3}=-k_{1}^{2}, \quad d_{4}=-k_{2}^{2}, \quad d_{5}=-\left(k_{1}-k_{2}\right)^{2} .
$$

The dimensionless function $G_{2}\left(n_{1}, n_{2}, n_{3}, n_{4}, n_{5}\right)$, whose expression we wish to obtain, is symmetric with respect to the changes $(1 \leftrightarrow 2,3 \leftrightarrow 4)$ and $(1 \leftrightarrow 3,2 \leftrightarrow 4)$. It is not possible to get a closed form for $G_{2}\left(n_{1}, n_{2}, n_{3}, n_{4}, n_{5}\right)$ in the general case, it is necessary to investigate each case separately.

For the cases where one of the indices $n_{i}$ is zero, one can easily find an expression for $G_{2}\left(n_{1}, n_{2}, n_{3}, n_{4}, n_{5}\right)$ in terms of the one-loop function $G_{1}\left(n_{1}, n_{2}\right)$ discussed in Sec. 3.2.1. Thus, if $n_{5}=0$, the diagram factors as a product of two one-loop diagrams, as illustrated in Fig. 14 and

$$
G_{2}\left(n_{1}, n_{2}, n_{3}, n_{4}, 0\right)=G_{1}\left(n_{1}, n_{2}\right) G_{1}\left(n_{3}, n_{4}\right) .
$$

Assuming now that $n_{1}=0$, then the first one-loop diagram in Fig. 15 will contribute with a factor $G_{1}\left(n_{3}, n_{5}\right)\left(-k_{2}^{2}\right)^{D / 2-n_{3}-n_{5}}$ and so the function $G_{2}\left(0, n_{2}, n_{3}, n_{4}, n_{5}\right)$ will be

$$
G_{2}\left(0, n_{2}, n_{3}, n_{4}, n_{5}\right)=G_{1}\left(n_{3}, n_{5}\right) G_{1}\left(n_{2}, n_{3}+n_{4}+n_{5}-D / 2\right) .
$$

In a similar way, the following expressions for the other cases can be evaluated

$$
\begin{aligned}
& G_{2}\left(n_{1}, 0, n_{3}, n_{4}, n_{5}\right)=G_{1}\left(n_{4}, n_{5}\right) G_{1}\left(n_{1}, n_{3}+n_{4}+n_{5}-D / 2\right), \\
& G_{2}\left(n_{1}, n_{2}, 0, n_{4}, n_{5}\right)=G_{1}\left(n_{1}, n_{5}\right) G_{1}\left(n_{4}, n_{1}+n_{2}+n_{5}-D / 2\right), \\
& G_{2}\left(n_{1}, n_{2}, n_{3}, 0, n_{5}\right)=G_{1}\left(n_{2}, n_{5}\right) G_{1}\left(n_{3}, n_{1}+n_{2}+n_{5}-D / 2\right) .
\end{aligned}
$$

However, if all the indices are positive, it is necessary to use integration by parts (or 
IBP). Applying the derivative $\partial / \partial k_{2}$ in the integrand of Eq. (3.28) (that we are calling $A$ ) times the term $\left(k_{2}-k_{1}\right)$ one gets

$$
\frac{\partial}{\partial k_{2}^{\mu}}\left[A\left(k_{2}^{\mu}-k_{1}^{\mu}\right)\right]=A\left[\frac{2 n_{2}}{d_{2}}\left(k_{2}+p\right)\left(k_{2}-k_{1}\right)+\frac{2 n_{4}}{d_{4}} k_{2}\left(k_{2}-k_{1}\right)+\frac{2 n_{5}}{d_{5}}\left(k_{2}-k_{1}\right)^{2}+D\right] .
$$

One can observe that the operation above is analogous to multiplying the integrand $A$ by ${ }^{45}$

$$
D-n_{2}-n_{4}-n_{5}+\frac{n_{2}}{d_{2}}\left(d_{1}-d_{5}\right)+\frac{n_{4}}{d_{4}}\left(d_{3}-d_{5}\right),
$$

where the definitions in Eq. (3.29) were adopted. The resulting integral vanishes, since the integral over total derivatives is zero. The action of these terms in the integrand can be expressed as a function of $G_{2}$. In order to do that, we introduce the following notation ${ }^{45}$

$$
\begin{aligned}
& \mathbf{1}^{ \pm} G_{2}\left(n_{1}, n_{2}, n_{3}, n_{4}, n_{5}\right)=G_{2}\left(n_{1} \pm 1, n_{2}, n_{3}, n_{4}, n_{5}\right), \\
& \mathbf{2}^{ \pm} G_{2}\left(n_{1}, n_{2}, n_{3}, n_{4}, n_{5}\right)=G_{2}\left(n_{1}, n_{2} \pm 1, n_{3}, n_{4}, n_{5}\right),
\end{aligned}
$$

and analogously for the others. Thus, the relation below can be written as

$$
G_{2}=\frac{n_{2} \mathbf{2}^{+}\left(\mathbf{5}^{-}-\mathbf{1}^{-}\right)+n_{4} \mathbf{4}^{+}\left(\mathbf{5}^{-}-\mathbf{3}^{-}\right)}{D-n_{2}-n_{4}-2 n_{5}} G_{2} .
$$

Hence, this formula can be applied in the function $G_{2}\left(n_{1}, n_{2}, n_{3}, n_{4}, n_{5}\right)$ that we want to obtain until one of the indices vanishes so Eqs. (3.30) to (3.32) can be employed.

This procedure is better understood with an example. Let us consider the function $G_{2}(1,1,1,1,1)$. Applying Eq. (3.35) to this function one gets

$$
\begin{aligned}
G_{2}(1,1,1,1,1)=\frac{1}{D-4}\left[G_{2}(1,2,1,1,0)-G_{2}(0,2,1,1,1)\right. & + \\
& \left.+G_{2}(1,1,1,2,0)-G_{2}(1,1,0,2,1)\right] .
\end{aligned}
$$

Since this function is symmetric with respect to the change $(1 \leftrightarrow 3,2 \leftrightarrow 4)$, as said before, it can be rewritten as

$$
G_{2}(1,1,1,1,1)=\frac{2}{D-4} g_{1}\left[G_{1}(2,1)-G_{1}(2,3-D / 2)\right]
$$

Defining $g_{2}=g_{1} G_{1}(1,2-D / 2)$ and applying Eq. (3.22), the relation below can be obtained

$$
G_{2}(1,1,1,1,1)=\frac{2}{D-4}\left[-(D-3) g_{1}+\frac{(3 D-8)(3 D-10)}{D-4} g_{2}\right] \text {. }
$$

Now that the master integrals method was introduced, it is possible to calculate the first-order QCD correction to the decay width of the Higgs boson into bottom quarks.

\subsection{3 $H \rightarrow b \bar{b}$ at Next-to-Leading Order}

The QCD corrections to the Higgs decay into $b$ quarks are due to the exchange of gluons. For the first-order correction, there are three diagrams that contribute, shown in 

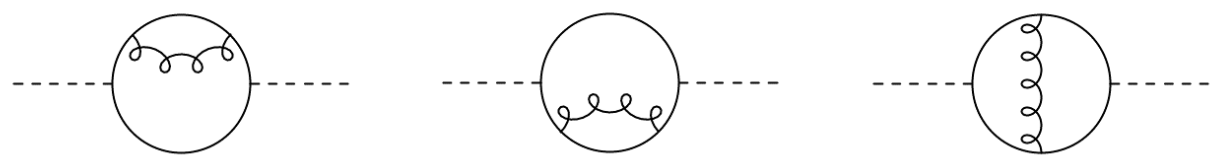

Figure 16 - Diagrams that contribute to the first-order correction.

Source: By the author.

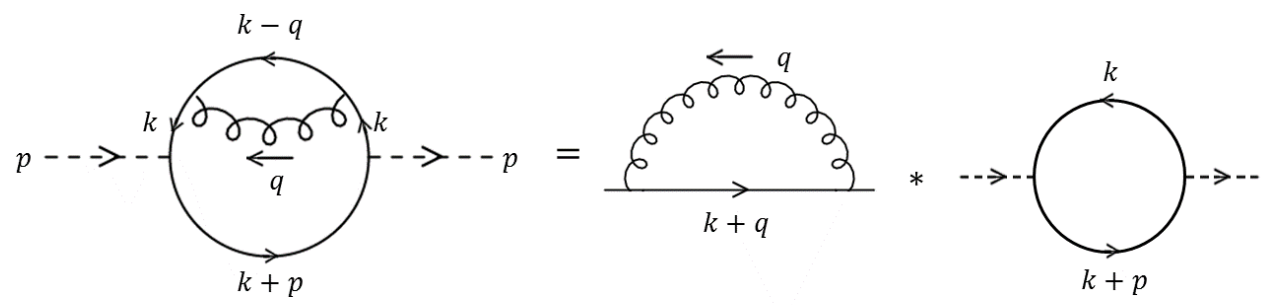

Figure 17 - Diagram for the first contribution.

Source: By the author.

Fig. 16, where some of them are not authentic two-loop diagrams. The result of the first two diagrams turns out to be the same, so it is sufficient to compute one of them and multiply the result by two.

We will start with the first diagram exhibited in Fig. 17. It is not a real two-loop diagram, because it factorizes as a product of a quark self-energy diagram and a one-loop diagram. To begin with, we will calculate using the master integral method the $b$-quark self-energy, $\Sigma(k)$, which, in the massless QCD, has the following form

$$
\Sigma(k)=\not k \Sigma_{V}\left(k^{2}\right) \text {. }
$$

Applying the Feynman rules and taking $\frac{1}{4} \operatorname{Tr}(\not k)$ on both sides of the equation the scalar function $\Sigma_{V}\left(k^{2}\right)$ is obtained

$$
\Sigma_{V}\left(k^{2}\right)=\frac{i \mu^{2 \epsilon} g_{s}^{2} \mu^{-2 \epsilon}}{(-k)^{2}} 4(2-D) \int \frac{\mathrm{d}^{D} q}{(2 \pi)^{D}} \frac{(q \cdot k)+k^{2}}{q^{2}(k+q)^{2}} .
$$

In order to write the expression above as a scalar integral, we will express it in terms of the parameters $d_{1}$ and $d_{2}$ shown in Sec. 3.2.1. The term $k^{2}$ is straightforward to obtain since $d_{2}=-k^{2}$, however for the first term $q \cdot k$ we have to remind that

$$
d_{1}=-(k+q)^{2}=d_{2}-2 k \cdot q-q^{2} \Longrightarrow k \cdot q=\frac{1}{2}\left(d_{2}-d_{1}-q^{2}\right) .
$$

Solving the integrals employing the master integral of Eq. (3.20), one finds the result below

$$
\Sigma_{V}\left(k^{2}\right)=-2 \mu^{2 \epsilon} g_{s}^{2} \mu^{-2 \epsilon}(D-2) \frac{\left(-k^{2}\right)^{D / 2-2}}{(4 \pi)^{D / 2}} g_{1} .
$$

Then we can evaluate the total expression for the diagram of Fig. 17. Hence we have

$$
i \Pi_{2}^{(1)}=-\int \frac{\mathrm{d}^{D} k}{(2 \pi)^{D}} \operatorname{Tr}\left[\left(-i m_{b}\right) \frac{i(\not k+\not p)}{(k+p)^{2}}\left(-i m_{b}\right) \frac{i \not k}{k^{2}}\left(-i \not k \Sigma_{V}\right) \frac{i \not k}{k^{2}}\right]=
$$




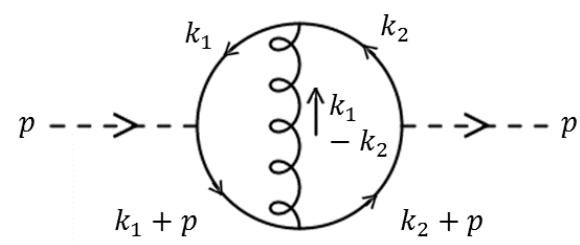

Figure 18 - Diagram for the second contribution.

Source: By the author.

$$
=-m_{b}^{2} \int \frac{\mathrm{d}^{D} k}{(2 \pi)^{D}} \quad \Sigma_{V} \frac{\operatorname{Tr}[(\not k+\not p) \not k]}{k^{2}(k+p)^{2}}=-m_{b}^{2} \int \frac{\mathrm{d}^{D} k}{(2 \pi)^{D}} \quad \Sigma_{V} \frac{4\left(p \cdot k+k^{2}\right)}{k^{2}(k+p)^{2}} .
$$

Substituting the bottom-quark self-energy given in Eq. (3.40), writing the integral in terms of $d_{1}$ and $d_{2}$ and solving it, we get the following answer for the first diagram

$$
\Pi_{2}^{(1)}=4 \mu^{2 \epsilon} g_{s}^{2} \mu^{-2 \epsilon} m_{b}^{2} \frac{\left(-p^{2}\right)^{D-3}}{(4 \pi)^{D}} \frac{2(D-2)^{2}}{D-4} g_{2} .
$$

We will at this point calculate the diagram responsible for the second contribution to the Higgs decay into $b \bar{b}$, whose diagram is shown in Fig. 18. In this case, it is a genuine two-loop diagram, whose integral in $D$-dimensions is given by

$$
i \Pi_{2}^{(2)}=-i \mu^{2 \epsilon} g_{s}^{2} \mu^{-2 \epsilon} m_{b}^{2} \int \frac{\mathrm{d}^{D} k_{1}}{(2 \pi)^{D}} \frac{\mathrm{d}^{D} k_{2}}{(2 \pi)^{D}} \frac{\operatorname{Tr}\left[\left(k_{1}+\not p\right) \gamma^{\mu}\left(k_{2}+\not p\right) k_{2} \gamma_{\mu} k_{1}\right]}{k_{1}^{2}\left(k_{1}+p\right)^{2}\left(k_{2}+p\right)^{2} k_{2}^{2}\left(k_{1}-k_{2}\right)^{2}} \operatorname{Tr}\left[\frac{1}{4} \lambda^{a} \lambda^{a}\right]
$$

where $\lambda^{a}$ are the Gell-Mann matrices that appear in the QCD vertex. Writing the function $\Pi_{2}^{(2)}$ in terms of $d_{1}, d_{2}, d_{3}, d_{4}$ and $d_{5}$, the non-vanishing integrals are

$$
\begin{aligned}
\Pi_{2}^{(2)}=8 \mu^{2 \epsilon} g_{s}^{2} \mu^{-2 \epsilon} m_{b}^{2} \int \frac{\mathrm{d}^{D} k_{1}}{(2 \pi)^{D}} \frac{\mathrm{d}^{D} k_{2}}{(2 \pi)^{D}} & \left\{\frac{(D-2)\left(d_{1} d_{4}+d_{2} d_{3}\right)}{d_{1} d_{2} d_{3} d_{4} d_{5}}+\right. \\
+ & \left.\frac{\left[2\left(d_{1}+d_{2}+d_{3}+d_{4}+p^{2}\right)+(D-4) d_{5}\right] p^{2}}{d_{1} d_{2} d_{3} d_{4} d_{5}}\right\} .
\end{aligned}
$$

All the integrals above are straightforward to compute using the two-loop master integral in Eq. (3.28) and the expressions for the function $G_{2}\left(n_{1}, n_{2}, n_{3}, n_{4}, n_{5}\right)$ given in Eqs. (3.30) to (3.32) and (3.37). After solving them one obtains as a function of $g_{1}$ and $g_{2}$

$$
\Pi_{2}^{(2)}=8 \mu^{2 \epsilon} g_{s}^{2} \mu^{-2 \epsilon} m_{b}^{2} \frac{\left(-p^{2}\right)^{D-3}}{(4 \pi)^{D}} \frac{(D-2)^{2}}{D-4}\left[\frac{2 D}{D-4} g_{2}-g_{1}^{2}\right] .
$$

Since all contributions were calculated, we can use the results for the two diagrams, given in Eqs. (3.42) and (3.43) to determine the total correlator $\Pi_{2}$ of the first-order correction. Reminding that the first diagram contributes twice, the result is

$$
\Pi_{2}=2 \Pi_{2}^{(1)}+\Pi_{2}^{(2)}=8 \alpha_{s} \mu^{2 \epsilon} m_{b}^{2} \frac{\left(-p^{2}\right)^{D-3}}{(4 \pi)^{D-1}} \frac{(D-2)^{2}}{D-4}\left[\frac{4(D-2)}{D-4} g_{2}-g_{1}^{2}\right] .
$$

Expanding the expression above near $\epsilon=0$ one finds 


$$
\begin{aligned}
\Pi_{2} & =\frac{1}{\epsilon} \frac{\alpha_{s}}{8 \pi^{3}} m_{b}^{2} p^{2}\left[\frac{3}{\epsilon}-6 \gamma_{E}+6 \ln 4 \pi+\frac{29}{2}+6 \ln \left(-\frac{p^{2}}{\mu^{2}}\right)\right]+ \\
& +\frac{\alpha_{s}}{32 \pi^{3}} m_{b}^{2} p^{2}\left\{227+24 \gamma_{E}^{2}-2 \pi^{2}+4 \ln 4 \pi(29+6 \ln 4 \pi)-4 \gamma_{E}(29+12 \ln 4 \pi)+\right. \\
& \left.-48 \zeta_{3}+24 \ln ^{2}\left(-\frac{p^{2}}{\mu^{2}}\right)+12 \ln \left(-\frac{p^{2}}{\mu^{2}}\right)\left[-29+12 \gamma_{E}-12 \ln 4 \pi\right]\right\}+\mathscr{O}(\epsilon) .
\end{aligned}
$$

The terms that contribute to the imaginary part, the logarithms, are still multiplied by a divergent term, $\frac{1}{\epsilon}$. Thus, before the Optical Theorem is applied, it is necessary to renormalize the theory so the result will be finite. In order to calculate the imaginary part, it is enough to renormalize the quark mass in the one-loop result, given in Eq. (3.25), which generates new terms of the order $\alpha_{s}$.

As already seen in Sec. 2.2, the renormalized mass and the renormalization constant up to first order in the $\overline{\mathrm{MS}}$ scheme are given by

$$
m=Z_{m} m^{R}, \quad Z_{m}=1-\frac{\alpha_{s}}{\pi \hat{\epsilon}}=1-\frac{\alpha_{s}}{\pi}\left[\frac{1}{\epsilon}-\gamma_{E}+\ln 4 \pi\right] .
$$

Then, the renormalized function $\Pi_{R}$ is written up to first order as

$$
\Pi_{R}=Z_{m}^{2} \Pi_{1}+\Pi_{2},
$$

where $\Pi_{1}$ and $\Pi_{2}$ are determined in Eqs. (3.25) and (3.44) respectively. Then, after substituting the renormalization constant and expanding it for $\epsilon \rightarrow 0$ we get

$$
\begin{aligned}
\Pi_{R} & =\frac{3 m_{b}^{2} p^{2}}{8 \pi^{2}}\left\{2-\gamma_{E}+\ln 4 \pi+\frac{1}{\epsilon}-\ln \left(-\frac{p^{2}}{\mu^{2}}\right)+\frac{\alpha_{s}}{\pi}\left[\frac{131}{12}+\frac{5}{3} \ln 4 \pi+\right.\right. \\
& -\ln ^{2} 4 \pi-\gamma_{E}\left(\frac{5}{3}+\gamma_{E}-2 \ln 4 \pi\right)+\frac{1}{\epsilon}\left(-\frac{1}{\epsilon}+\frac{5}{6}+2 \gamma_{E}-2 \ln 4 \pi\right)+ \\
& \left.\left.-\frac{17}{3} \ln \left(-\frac{p^{2}}{\mu^{2}}\right)+\ln ^{2}\left(-\frac{p^{2}}{\mu^{2}}\right)-4 \zeta_{3}\right]\right\}+\mathscr{O}\left(\alpha_{s}^{2}\right),
\end{aligned}
$$

where we adopted $N_{c}=3$. As one can notice, the logarithm terms are no longer divergent when $\epsilon \rightarrow 0$.

With the result above, it is possible now to compute the first order correction to the decay width of the Higgs boson into bottom quarks. Taking the imaginary part of the expression above, we get in the limit $\left(m_{b} / m_{H}\right)^{2} \rightarrow 0$

$$
\Gamma(H \rightarrow b \bar{b})=\frac{3}{8 \pi v^{2}} m_{b}^{2} p^{2}\left[1+\frac{17}{3 \pi} \alpha_{s}+\mathscr{O}\left(\alpha_{s}^{2}\right)\right],
$$


which agrees exactly with the results in the literature. ${ }^{6,7,47,48}$ The calculations done in this section are the basis of the methods employed at higher orders. However, the computations of the three-loop or higher corrections are more complicated and totally out of the scope of this dissertation. The difficulty of these calculations is evident in the interval between the computation of the coefficients of the imaginary part: the first-order correction was determined in $1980^{47,48}$ and the second-order in $1990^{49}$ after 10 years, the third-order coefficient was obtained in $1997^{8}$ and the fourth-order 10 years later ${ }^{6}$ in 2006. As of today, the result at $\alpha_{s}^{5}$ remains unknown. Now we will present and discuss the state-of-the-art of this calculation.

\subsection{Scalar Correlator at Higher Orders}

As already mentioned, the decay width of the Higgs boson into the pair $b \bar{b}$ is known up to fourth order in $\mathrm{QCD}^{6,7}$ for $\left(m_{b} / m_{H}\right)^{2} \rightarrow 0$ and is related to the imaginary part of the scalar correlator $\Pi$ due to the Optical Theorem. This correlator, that is not directly associated with any physical quantity, is defined in Eq. (3.7) and its purely perturbative expansion in terms of the strong coupling is given by the following general form ${ }^{41}$

$$
\Pi(s)=-\frac{N_{c}}{8 \pi^{2}} m^{2} s \sum_{n=0}^{\infty} a_{s}^{n}(\mu) \sum_{k=0}^{n+1} d_{n, k} L^{k},
$$

where $s \equiv p^{2}$. The quark mass $m$ and the QCD coupling $a_{s}$ are renormalized at the scale $\mu$, which appears in $L \equiv \ln \left(-s / \mu^{2}\right)^{\S}$.

The coefficients $d_{n, 0}$ depend on the conventions related to the renormalization procedure employed and do not contribute in any physical quantity. Furthermore, the coefficients $d_{n, k}$, with $k>1$, can be obtained from the RGE of the scalar correlator, which is given by

$$
-\mu \frac{\mathrm{d} \Pi}{\mathrm{d} \mu}=\left[2 \frac{\partial}{\partial L}+m \gamma_{m}\left(a_{s}\right) \frac{\partial}{\partial m}+\beta\left(a_{s}\right) \frac{\partial}{\partial a_{s}}\right] \Pi=m^{2} s \gamma_{S S}\left(a_{s}\right),
$$

with $\gamma_{S S}$ being the scalar-scalar anomalous dimension. ${ }^{8}$ Hence the only independent coefficients for each perturbation order are $d_{n, 1}$, whose expressions in terms of the number of quark flavors $N_{f}$ and for $N_{c}=3$ are, up to fourth order, ${ }^{6,7}$

$$
\begin{aligned}
d_{0,1} & =1, \quad d_{1,1}=\frac{17}{3}, \quad d_{2,1}=\frac{10801}{144}-\frac{39}{2} \zeta_{3}+\left(-\frac{65}{24}+\frac{2}{3} \zeta_{3}\right) N_{f}, \\
d_{3,1} & =\frac{6163613}{5184}-\frac{109735}{216} \zeta_{3}+\frac{815}{12} \zeta_{5}- \\
& -\left(\frac{46147}{486}-\frac{262}{9} \zeta_{3}+\frac{5}{6} \zeta_{4}+\frac{25}{9} \zeta_{5}\right) N_{f}+\left(\frac{15511}{11664}-\frac{1}{3} \zeta_{3}\right) N_{f}^{2}, \\
d_{4,1} & =\frac{10811054729}{497664}-\frac{3887351}{324} \zeta_{3}+\frac{458425}{432} \zeta_{3}^{2}+\frac{265}{18} \zeta_{4}+\frac{373975}{432} \zeta_{5}-\frac{1375}{32} \zeta_{6}-\frac{178045}{768} \zeta_{7}+
\end{aligned}
$$

\footnotetext{
${ }^{\S}$ Sometimes we will omit the dependence of $\alpha_{s}$ and $m$ on the renormalization scale.
} 


$$
\begin{aligned}
& -\left(\frac{1045811915}{373248}-\frac{5747185}{5148} \zeta_{3}+\frac{955}{16} \zeta_{3}^{2}+\frac{955}{16} \zeta_{4}-\frac{41215}{432} \zeta_{5}-\frac{2875}{288} \zeta_{6}+\frac{178045}{768} \zeta_{7}\right) N_{f}+ \\
& +\left(\frac{220313525}{2239488}-\frac{11875}{432} \zeta_{3}+\frac{5}{6} \zeta_{3}^{2}+\frac{25}{96} \zeta_{4}-\frac{5015}{432} \zeta_{5}\right) N_{f}^{2}- \\
& -\left(\frac{520771}{559872}-\frac{65}{432} \zeta_{3}-\frac{1}{144} \zeta_{4}-\frac{5}{18} \zeta_{5}\right) N_{f}^{3}
\end{aligned}
$$

The first two coefficients, $d_{0,1}$ and $d_{1,1}$, were calculated by us in the last section in Eqs. (3.26) and (3.48) respectively. The numerical values of these coefficients for $N_{f}=5$ are

$$
d_{1,1}=5.6667, \quad d_{2,1}=42.032, \quad d_{3,1}=353.229, \quad d_{4,1}=3512.2 .
$$

In our case, the observable is related to the imaginary part of the correlator $\Pi(s)$. Setting the renormalization scale to $\mu^{2}=s \equiv m_{H}^{2}$, so the logarithms are summed, the general perturbative expansion for the imaginary part is

$$
\operatorname{Im} \Pi(s)=\frac{N_{c}}{8 \pi} m^{2} s \sum_{n=0}^{\infty} a_{s}^{n}\left(m_{H}\right) \sum_{k=0}^{[n / 2]} d_{n, 2 k+1}(i \pi)^{2 k},
$$

with $[x]$ representing the least integer not greater than $x$. Therefore, for $N_{f}=5$, the coefficients up to fourth order in QCD are

$$
\begin{aligned}
\operatorname{Im} \Pi(s) & =\frac{N_{c}}{8 \pi} m^{2} s \sum_{n=0}^{\infty} c_{n} a_{s}^{n}= \\
& =\frac{N_{c}}{8 \pi} m^{2} s\left[1+5.6667 a_{s}+29.1467 a_{s}^{2}+41.7576 a_{s}^{3}-825.747 a_{s}^{4}+\ldots\right],
\end{aligned}
$$

whose results up to first order were previously computed at Sec. 3.2.3. The exact calculations for the higher-order corrections are even harder to compute: the interval between the calculation of the third- and fourth-order coefficients was 10 years. ${ }^{6,8}$ It does not seem possible to compute the fifth-order coefficient soon, because of the arduous calculations due to the gluon self-interactions. Hence, it is important to develop other techniques to obtain estimates for the higher-order corrections, as we will do in this work.

The perturbative expansion of $\operatorname{Im} \Pi$ can be written without the influence of the running quark mass, i.e., in terms of the invariant mass $\widehat{m}$ defined in Eq. (2.63). In this way, re-expanding the imaginary part in $a_{s}$, one finds

$$
\begin{aligned}
\operatorname{Im} \Pi(s) & =\frac{N_{c}}{8 \pi^{2}} \widehat{m}^{2} s\left(\alpha_{s}\right)^{2 \gamma_{m}^{(1)} / \beta_{1}}\left\{1+\sum_{n=1}^{\infty} \widehat{c}_{n} a_{s}^{n}\right\}= \\
= & \frac{N_{c}}{8 \pi} \widehat{m}^{2} s\left(\alpha_{s}\right)^{2 \gamma_{m}^{(1)} / \beta_{1}}\left[1+8.0176 a_{s}+46.8521 a_{s}^{2}+138.992 a_{s}^{3}-571.886 a_{s}^{4}+\ldots\right]
\end{aligned}
$$

whose numerical values were calculated for $N_{f}=5$. A negative coefficient appears for the first time in the term of order $a_{s}^{4}$ of the imaginary part and, as we will see in the next 
chapter, it may indicate the influence of an UV renormalon. Since the imaginary part of the scalar correlator is a physical observable, it satisfies a homogeneuos RGE and the logarithms can be reconstructed from it.

Another quantity that is directly related to a physical observable is the second derivative of the scalar correlator with respect to $s$, since it removes the two scheme-dependent constants. Its perturbative expansion is given by

$$
\Pi^{\prime \prime}(s)=-\frac{N_{c}}{8 \pi^{2}} \frac{m^{2}}{s} \sum_{n=0}^{\infty} a_{s}^{n}(\mu) \sum_{k=1}^{n+1} d_{n, k} k\left[L^{k-1}+(k-1) L^{k-2}\right] .
$$

In order to sum all the logarithms in the expression of the second derivative, we choose the scale $\mu^{2}=-s$ and get the following expression

$$
\Pi^{\prime \prime}(s)=-\frac{N_{c}}{8 \pi^{2}} \frac{m^{2}}{s}\left[1+\sum_{n=1}^{\infty} a_{s}^{n}\left(d_{n, 1}+2 d_{n, 2}\right)\right]=-\frac{N_{c}}{8 \pi^{2}} \frac{m^{2}}{s}\left[1+\sum_{n=1}^{\infty} r_{n} a_{s}^{n}\right],
$$

whose coefficients for $N_{f}=5$ are

$$
\Pi^{\prime \prime}(s)=-\frac{N_{c}}{8 \pi^{2}} \frac{m^{2}}{s}\left[1+3.6667 a_{s}+12.8098 a_{s}^{2}+39.6839 a_{s}^{3}+153.955 a_{s}^{4}+\ldots\right] .
$$

Similarly, one can write the second derivative in terms of the invariant mass $\widehat{m}$ as

$$
\begin{aligned}
& \Pi^{\prime \prime}(s)=-\frac{N_{c}}{8 \pi^{2}} \frac{\widehat{m}^{2}}{s}\left(\alpha_{s}\right)^{2 \gamma_{m}^{(1)} / \beta_{1}}\left[1+\sum_{n=1}^{\infty} \widehat{r}_{n} a_{s}^{n}\right]= \\
& \quad=-\frac{N_{c}}{8 \pi^{2}} \frac{\widehat{m}^{2}}{s}\left(\alpha_{s}\right)^{2 \gamma_{m}^{(1)} / \beta_{1}}\left[1+6.0176 a_{s}+25.8132 a_{s}^{2}+89.7442 a_{s}^{3}+323.587 a_{s}^{4}+\ldots\right]
\end{aligned}
$$

where the coefficients were calculated for $N_{f}=5$ and, as we can see, all the known ones are positive. Due to the fact that the second derivative is a physical observable, as the imaginary part, it also satisfies a homogeneous RGE and its logarithmic terms can be resummed from it.

It is important to remember that the perturbative series of the imaginary part, the second derivative and the scalar correlator are divergent and expected to be asymptotic. We will discuss this divergence in the next chapter and introduce the Borel transform, which is an effective method of working with series of this type. 



\section{ASYMPTOTIC SERIES AND PADÉ APPROXIMANTS}

\subsection{Divergent Series and Renormalons}

Until the late 40s it was the general believe that the perturbative series were convergent at least for sufficiently small values of the coupling. However, in 1952 Dyson $^{50}$ argued that the series in $e^{2}$ in QED should be divergent because negative values for this coupling produce a theory with unstable vacuum. This is know as the Dyson argument. A few years later Lipatov concluded that in scalar field theories of the type $\lambda \phi^{n}$, for even $n \geq 4$, the perturbative series are asymptotic and are factorially divergent. ${ }^{51}$ The arguments used could also be generalized for other quantum field theories as QED and the Yang-Mills.

Thus, in general, the perturbative series in a realistic quantum field theory is divergent. In addition, there are at least two sources for the factorial divergence in Yang-Mills theory, which is the case of QCD, that are described below ${ }^{9,34,52}$

* Instanton-antiinstanton singularities: classical solutions of the QCD equations of motion. They are associated with the number of diagrams, which grows factorially with the order of the perturbative expansion.

* Renormalons: single diagrams that contribute a factor $n$ ! at order $n$ to the amplitude.

Here we are concerned with divergences of the second type and, in order to study them, we will consider an example that has become standard: the Adler function, defined as

$$
D\left(Q^{2}\right)=-4 \pi^{2} Q^{2} \frac{\mathrm{d} \Pi\left(Q^{2}\right)}{\mathrm{d} Q^{2}},
$$

where $\Pi$ is related to the correlation function $\Pi_{\mu \nu}$ of two vector currents $\left(j_{\mu}=\bar{q} \gamma_{\mu} q\right)$ of massless quarks by

$$
\Pi_{\mu \nu}(q)=i \int \mathrm{d}^{4} x \mathrm{e}^{-i q x}\left\langle 0\left|T\left[j_{\mu}(x) j_{\nu}(0)\right]\right| 0\right\rangle=\left(q_{\mu} q_{\nu}-q^{2} g_{\mu \nu}\right) \Pi\left(Q^{2}\right),
$$

where $q^{2}=-Q^{2}$ is the external momentum. This correlator appears in the calculation of the vacuum polarization of the photon, for example.

Corrections to this correlator can be obtained using the methods described in Ch. 3. An important class of diagrams is the one with a single gluon line replaced by a fermion bubble chain. We can, at this point, calculate the contributions due to the fermion bubble chains to the Adler function, illustrated at Fig. 19. Each renormalized fermion loop, which is gauge invariant, is given by ${ }^{9}$

$$
-\beta_{0 f} \alpha_{s}\left[\ln \left(-\frac{k^{2}}{\mu^{2}}\right)+C-\frac{5}{3}\right],
$$




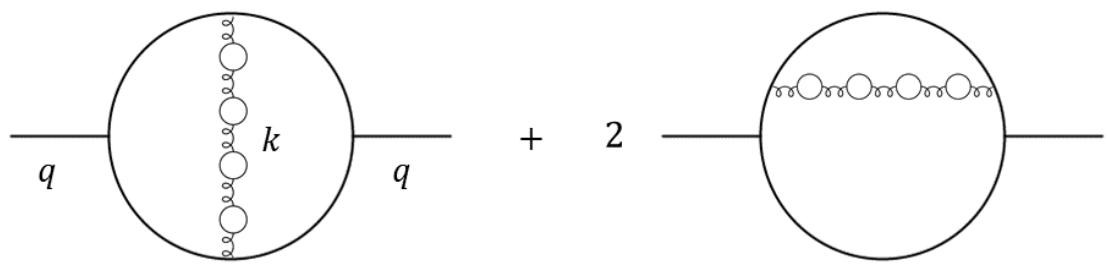

Figure 19 - Corrections to the Adler function.

Source: By the author.

where $\beta_{0 f}$ is the fermionic contribution to the one-loop $\beta$ function and $C$ is related to the renormalization scheme. It is necessary to sum over all the fermion loops and integrate over the gluon momentum $k$, which, for $k_{E}^{2}=-k^{2}$, results in the $\overline{\mathrm{MS}}$ scheme $(C=0)^{9}$

$$
D\left(Q^{2}\right)=\sum_{n=0}^{\infty} \alpha_{s}\left(Q^{2}\right) \int_{0}^{\infty} \frac{\mathrm{d} k_{E}^{2}}{k_{E}^{2}} F\left(k_{E}^{2}, Q^{2}\right)\left[\frac{\beta_{1}}{2 \pi} \alpha_{s}\left(Q^{2}\right) \ln \left(\frac{k_{E}^{2} \mathrm{e}^{-5 / 3}}{Q^{2}}\right)\right]^{n} .
$$

The function $F\left(k_{E}^{2}, Q^{2}\right)$ is a scheme- and scale-independent distribution function of loop momentum obtained from the Borel transform of the series, whose exact result was calculated by Neubert. ${ }^{53}$ We substituted $\beta_{0 f}$ with $\frac{\beta_{1}}{2 \pi}$, a procedure denominated nonabelianization, and it will be clear later the reason to do it. The equation above is essentially a two-loop integral, with the integrals over the external quark-loop momentum and the solid angle associated with $k$ already done. The logarithm inside the integral appears due to writing the strong coupling $\alpha_{s}$ at the scale $Q^{2}$ using only the one-loop beta function, which is given in Eq. (2.66).

One can now analyze the integral of Eq. (4.4) in the limits $k_{E}^{2} \gg Q^{2}$ (high energies) and $k_{E}^{2} \ll Q^{2}$ (low energies). We will start with the high-energy case, where the distribution function $F\left(k_{E}^{2}, Q^{2}\right)$ can be approximated by ${ }^{9,53}$

$$
F_{\mathrm{UV}}\left(k_{E}^{2}, Q^{2}\right)=\left[A \ln \left(\frac{k_{E}^{2}}{Q^{2}}\right)+B\right] \frac{Q^{2}}{k_{E}^{2}},
$$

where $A$ and $B$ are constants. Replacing the expression above in Eq. (4.4) and with the change of variable $t=k_{E}^{2} / Q^{2}$ one finds

$$
D_{\mathrm{UV}}\left(Q^{2}\right)=\sum_{n=0}^{\infty} \int_{1}^{\infty} \frac{\mathrm{d} t}{t^{2}}(A \ln t+B)\left(-\frac{\beta_{1}}{2 \pi}\right)^{n} \alpha_{s}^{n+1}\left(Q^{2}\right) \ln ^{n}(t) .
$$

Performing another change of variable, $t=\mathrm{e}^{y}$, one gets

$$
\begin{aligned}
D_{\mathrm{UV}}\left(Q^{2}\right) & =\sum_{n=0}^{\infty}\left(-\frac{\beta_{1}}{2 \pi}\right)^{n} \alpha_{s}^{n+1}\left(Q^{2}\right) \int_{0}^{\infty} \mathrm{d} y(A y+B) y^{n} \mathrm{e}^{-y}= \\
& =\sum_{n=0}^{\infty}\left(-\frac{\beta_{1}}{2 \pi}\right)^{n} \alpha_{s}^{n+1}\left(Q^{2}\right)(A n+A+B) n !
\end{aligned}
$$


One can notice that the series above is divergent because of the factor $n$ !. No matter how small the coupling is, eventually, the factorial growth of the coefficients dominates and the series diverges. This divergence is called ultraviolet (UV) renormalon due to its origin from high energies. It is also important to observe that this series is sign-alternating.

Let us consider now the low-energy limit. The function $F\left(k_{E}^{2}, Q^{2}\right)$ takes the form ${ }^{9,53}$

$$
F_{\mathrm{IR}}\left(k_{E}^{2}, Q^{2}\right)=E\left(\frac{k_{E}^{2}}{Q^{2}}\right)^{2},
$$

where $E$ is a constant. Substituting $F_{\mathrm{IR}}\left(k_{E}^{2}, Q^{2}\right)$ in Eq. (4.4) and changing the variable as $k_{E}^{2} / Q^{2}=\mathrm{e}^{-y}$, the expression for the Adler function is

$$
\begin{aligned}
D_{\mathrm{IR}}\left(Q^{2}\right) & =\sum_{n=0}^{\infty}\left(-\frac{\beta_{1}}{2 \pi}\right)^{n} \alpha_{s}^{n+1}\left(Q^{2}\right) \int_{0}^{\infty} \mathrm{d} y E(-y)^{n} \mathrm{e}^{-2 y}= \\
& =\frac{E}{2} \sum_{n=0}^{\infty}\left(\frac{\beta_{1}}{4 \pi}\right)^{n} \alpha_{s}^{n+1}\left(Q^{2}\right) n ! .
\end{aligned}
$$

This series is also divergent for all values of $\alpha_{s}$, however it is a fixed-sign one. The factorial divergence in this case is known as infrared (IR) renormalon, since it is derived from the low-energy limit.

The calculations demonstrated here are not complete, since the strong coupling was written in terms of the one-loop $\beta$ function and only a small part of the higher-order diagrams are considered, although all of them are present in the QCD calculations. Regarding the one-loop approximation for the running of the strong coupling $\alpha_{s}$, the $\beta$ function in QCD is very convergent, and including more loops does not lead to qualitative changes. Thus, the results obtained here are in essence preserved in QCD, although some differences appear, as we will see later. ${ }^{9}$

\subsection{Asymptotic Series and Borel Transform}

As we discussed in Sec. 4.1, the perturbative series in $\alpha_{s}$ in QFT is generally divergent and at best asymptotic. ${ }^{50}$ An important property of an asymptotic series is to provide a good approximation for a given function when truncated after a finite number of terms.

The main characteristic of this kind of series is that its partial sum up to a certain finite order approaches a given function, showing an asymptotic behavior, and then it starts to diverge. Let $f(\alpha)$ be a given function, so a power series $R(\alpha)=\sum_{k=0}^{\infty} r_{k} \alpha^{k}$ is an asymptotic expansion of $f(\alpha)$ if, for a fixed $N$, it converges to the function $f(\alpha)$ in the limit $\alpha \rightarrow 0$; i.e., for all $N>0^{54}$

$$
\lim _{\alpha \rightarrow 0} \frac{1}{\alpha^{N}}\left[f(\alpha)-\sum_{k=0}^{N} r_{k} \alpha^{k}\right]=0 .
$$


When increasing $N$ enough for fixed $\alpha$, the partial sum of the series $R(\alpha)$ will first exhibit an asymptotic behavior, approaching the true value, and eventually will diverge. The coefficients $r_{k}$ of an asymptotic series in a theory as QCD are factorially divergent, and, for a given order $k$, they behave as ${ }^{9,52}$

$$
r_{k} \approx c_{k} a^{k} k ! k^{b}
$$

where $a$ and $b$ are constants and $c_{k}$ is a numerical coefficient of order one.

As mentioned before, series that arise from QFT are usually asymptotic, and it is convenient to employ the Borel transform method. The Borel transform of a series is essentially the inverse Laplace transform and it suppresses, at least partially, the factorial divergence of the series.

Let $R=\sum_{k=0}^{\infty} r_{k} \alpha^{k+1}$ be an asymptotic expansion of an observable $R$. After multiplying and dividing each coefficient by $k$, reminding that $k !=\int_{0}^{\infty} \mathrm{e}^{-t} t^{k} \mathrm{~d} t$, we find

$$
R=\sum_{k=0}^{\infty} \int_{0}^{\infty} \mathrm{e}^{-t} t^{k} \frac{r_{k}}{k !} \alpha^{k+1} \mathrm{~d} t .
$$

Making the change of variable $u=\alpha t$, we obtain the following result

$$
R=\int_{0}^{\infty} \sum_{k=0}^{\infty} \frac{r_{k}}{k !} u^{k} \mathrm{e}^{-u / \alpha} \mathrm{d} u=\int_{0}^{\infty} B[R](u) \mathrm{e}^{-u / \alpha} \mathrm{d} u,
$$

where $B[R](u)$ is the Borel transform of $R$ defined as $^{9}$

$$
B[R](u)=\sum_{k=0}^{\infty} \frac{r_{k}}{k !} u^{k} .
$$

Thus it is possible to notice that the factorial divergence of the coefficient $r_{k}$ is now eliminated through the term $(k !)^{-1}$. The Borel transform can be used as a generator of the original function $R$. Knowing the coefficients of $B[R]$, given in Eq. (4.14), we can multiply the $k$ th coefficient by $k$ ! recovering the coefficients of $R$.

When the Borel transform does not have singularities on the positive axis of $u$, the integral shown in Eq. (4.13), denominated Borel integral, can be done regularly and it will give the value of $R$ summed in the Borel sense. In these cases, we say that the function is Borel summable. On the other hand, when there are singularities on the positive real axis of $u$, we must encircle the poles to calculate the integral, which can be done in more than one way. Because of that there is an ambiguity of the Borel integral and we say that the function is not Borel summable.

The singularities of the Borel transform on the complex plane of $u$, which in our case are the renormalons of perturbation theory introduced in Sec. 4.1, govern the behavior of the perturbative series at intermediate and higher orders. ${ }^{9}$ If the poles are located on the positive real axis, they are the infrared (IR) renormalons and the series will be fixed-sign. But when they are on the negative real axis, the series will be sign-alternated and the 
renormalons are the ultraviolet (UV) ones $\mathbb{I}$.

In order to observe the relation between the renormalons of Sec. 4.1 and the singularities of the Borel transform, we can now calculate the Borel transform of the Adler function. Let us start with the high-energy limit, where the Adler function, which is a sign-alternating series, is given by Eq. (4.7). Using Eq. (4.14), we obtain the following expression

$$
B\left[D_{\mathrm{UV}}\right]=\sum_{n=0}^{\infty}\left(-\frac{\beta_{1}}{2 \pi}\right)^{n}(A n+A+B) t^{n},
$$

and performing the change of variable $u=\frac{\beta_{1} t}{2 \pi}$

$$
B\left[D_{\mathrm{UV}}\right](u)=\sum_{n=0}^{\infty}(-1)^{n}(A n+A+B) u^{n}=\frac{A}{(1+u)^{2}}+\frac{B}{1+u} .
$$

As we can see, in this limit the Borel transform has a pole at $u=-1$, and if we considered the full expression of $F\left(k_{\mathrm{E}}^{2}, Q^{2}\right)$, there would be poles at $u=-1,-2,-3, \ldots{ }^{9}$ Thus, the UV renormalon is a singularity on the negative real axis of the Borel transform.

Considering now the Adler function in the low-energy limit, which is a fixed-sign series indicated in Eq. (4.9), we can calculate the Borel transform below, where $u=\frac{\beta_{1} t}{2 \pi}$,

$$
B\left[D_{\mathrm{IR}}\right](u)=\sum_{n=0}^{\infty} \frac{E}{2}\left(\frac{u}{2}\right)^{n}=\frac{E}{u-2} .
$$

At low energies, the Borel transform has a single pole at $u=2$ and if the full expression of the distribution function $F\left(k_{\mathrm{E}}^{2}, Q^{2}\right)$ was considered, the poles $u=3,4,5, \ldots$ would also be present. ${ }^{9}$ The IR renormalons are then singularities on the positive axis of the Borel transform. The use of the naive non-abelianization, the procedure that changes the fermionic contribution to the one-loop $\beta$ function for $\beta_{1}$, is very important since it inverts the position of the poles, because $\beta_{0 f}$ and $\beta_{1}$ have different signs, and incorporates, albeit effectively, non-abelian effects in the result.

We will consider now, as an example, the following generic divergent series

$$
R(\alpha)=\sum_{k=0}^{\infty} \alpha^{k+1} k ! \frac{r}{p^{k+1}},
$$

whose Borel transform can be computed as

$$
B[R](u)=\sum_{k=0}^{\infty} u^{k} \frac{r}{p^{k+1}}=\frac{r}{p-u} .
$$

It is possible to see that, if the Borel transform has an UV renormalon, i.e., $p<0$, the series $R$ is sign-alternating; and if the renormalon is an IR one $(p>0)$ then the series is fixed-sign. Let us now analyze the behavior of two specific cases, which are

$$
f_{1}(\alpha)=\sum_{k=0}^{\infty} \alpha^{k+1} k ! \quad \text { and } \quad f_{2}(\alpha)=\sum_{k=0}^{\infty}(-\alpha)^{k+1} k ! .
$$

$\overline{\mathbb{I}}$ There are rare exceptions to this rule, however they do not appear in this dissertation. 


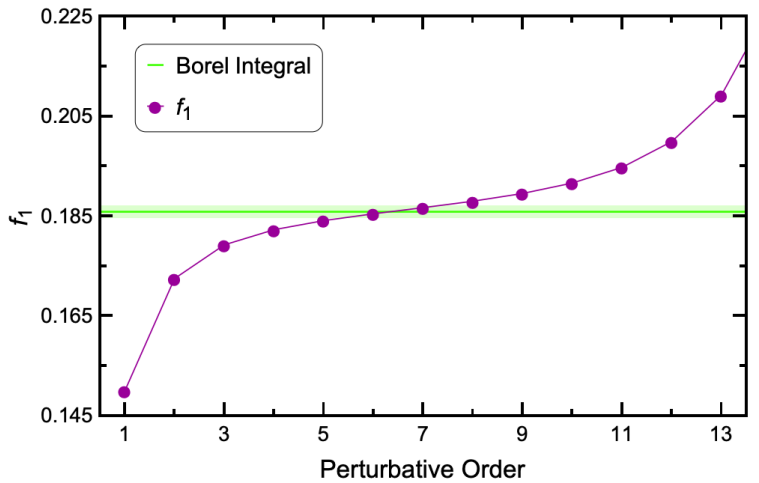

Figure $20-B\left[f_{1}\right](u)$ given in Eq. (4.21) adopting $\alpha=0.15$.

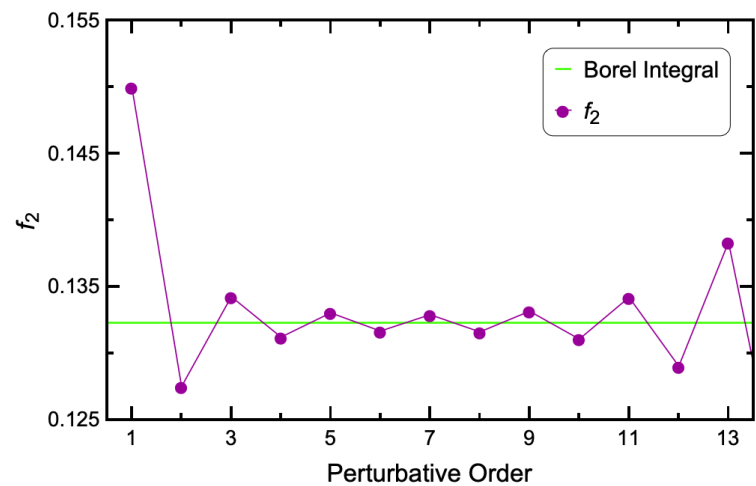

Figure $21-B\left[f_{2}\right](u)$ given in Eq. (4.21) adopting $\alpha=0.15$.

Source: By the author.

The Borel transforms of the functions $f_{1}$ and $f_{2}$ are indicated below

$$
B\left[f_{1}\right](u)=\frac{1}{1-u} \quad \text { and } \quad B\left[f_{2}\right](u)=\frac{1}{1+u} .
$$

The first function $f_{1}$ is a fixed-sign series since it has an IR renormalon and $f_{2}$ is a sign-alternating series due to its UV renormalon. This behavior can be seen order by order in Figs. 20 and 21. The green lines in both graphics are the Borel integrals, calculated using $\alpha=0.15$, and the light-green shaded area in Fig. 20 is the Borel ambiguity: the integral ambiguity divided by $\pi$. Fig. 21 does not have an ambiguity since $B\left[f_{2}\right](u)$ is Borel summable. Analyzing Figs. 20 and 21, we can notice that the first function $f_{1}$ starts to diverge at the eighth-order and the other one, $f_{2}$, diverges at the eleventh-order. It is important to emphasize that the higher the value of $\alpha$ the earlier the divergence appears.

A highly used method to assign a value for an asymptotic series is to truncate it in the term that provides the lowest contribution. This mechanism is denominated Optimal Truncation and there is no guarantee of its effectiveness, however it is in general empirically valid. ${ }^{55}$ As an example, let us consider again the functions $f_{1}$ and $f_{2}$ given in Eq. (4.20). By examining Figs. 20 and 21 the last term we should use to sum those series is the seventh-order coefficient of both functions.

One can, at this point, analyze what happens when the Borel transform has more than one pole on the real axis. Let $p_{1}$ and $p_{2}$ be two poles where $\left|p_{1}\right|>\left|p_{2}\right|$ and the residues are the same. From Eq. (4.18), it is possible to identify the contribution of the poles to the $k$ th coefficient of the sum as $p_{1}^{-(k+1)}+p_{2}^{-(k+1)}$. For large values of $k$, the contribution of $p_{1}$ is subdominant to $p_{2}$, since $\left|p_{1}\right|>\left|p_{2}\right|$. Hence, the poles closer to the origin dominate the series behavior at intermediate and large orders. 


\subsection{Padé Approximants}

In this work, we aim at analyzing the Borel transform of the perturbative series in QCD for the Higgs decay into bottom quarks using the mathematical method of Padé approximants. Therefore, in this section we will introduce the most important concepts about Padé approximants.

The Padé approximant (PA) is a rational approximation of a function whose Taylor series is known. ${ }^{13}$ The PAs are largely suitable for the application to functions with singularities (as the Borel transforms which we will study), for the reason that they also have poles, so they are capable of providing a good approximation near the singularities. ${ }^{12}$

Let us consider a function $f(z)$ whose series expansion in the complex plane around $z=0$ is given by

$$
f(z)=\sum_{n=0}^{\infty} f_{n} z^{n} .
$$

A Padé approximant to the function $f(z),{ }^{11}$ denoted as $P_{N}^{M}(z)$, is defined as the ratio of two polynomials $Q_{M}(z)$ and $R_{N}(z)$ of order $M$ and $N$ respectively, where the definition $R_{N}(0)=1$ is employed without loss of generality

$$
P_{N}^{M}(z)=\frac{Q_{M}(z)}{R_{N}(z)}=\frac{a_{0}+a_{1} z+\cdots+a_{M} z^{M}}{1+b_{1} z+\cdots+b_{N} z^{N}} .
$$

The PA makes a "contact" of order $M+N$ with the expansion of the function around $z=0 ;{ }^{56}$ which means that, when $P_{N}^{M}(z)$ is expanded around $z=0$, it will reproduce exactly the first $M+N$ coefficients $f_{n}$.

The coefficients $a_{0}, \ldots, a_{M}, b_{1}, \ldots, b_{N}$ are determined performing a matching between the Padé expansion and the expansion of Eq. (4.22) up to order $M+N$. In this way, the Padé can be reconstructed and used to make predictions about the coefficients with order higher than $M+N$. This procedure is better illustrated with a concrete example: we will calculate the Padé $P_{1}^{1}$ to the function $f(z)=\frac{1-z}{(1+4 z)^{2}}$. The Taylor expansion of $f(z)$ is

$$
f(z)=\frac{1-z}{(1+4 z)^{2}} \approx 1-9 z+56 z^{2}-304 z^{3}+1536 z^{4}+\ldots
$$

And expanding now $P_{1}^{1}$

$$
P_{1}^{1}(z)=\frac{a_{0}+a_{1} z}{1+b_{1} z} \approx a_{0}+\left(a_{1}-a_{0} b_{1}\right) z+\left(a_{0} b_{1}^{2}-a_{1} b_{1}\right) z^{2}+\ldots
$$

Matching the first three coefficients of $f(z)$ and $P_{1}^{1}$ the following values for the coefficients $a_{0}, a_{1}$ and $b_{1}$ can be obtained

$$
a_{0}=1, \quad a_{1}=-\frac{25}{9}, \quad b_{1}=\frac{56}{9},
$$

which can now be used to reconstruct the Padé as 


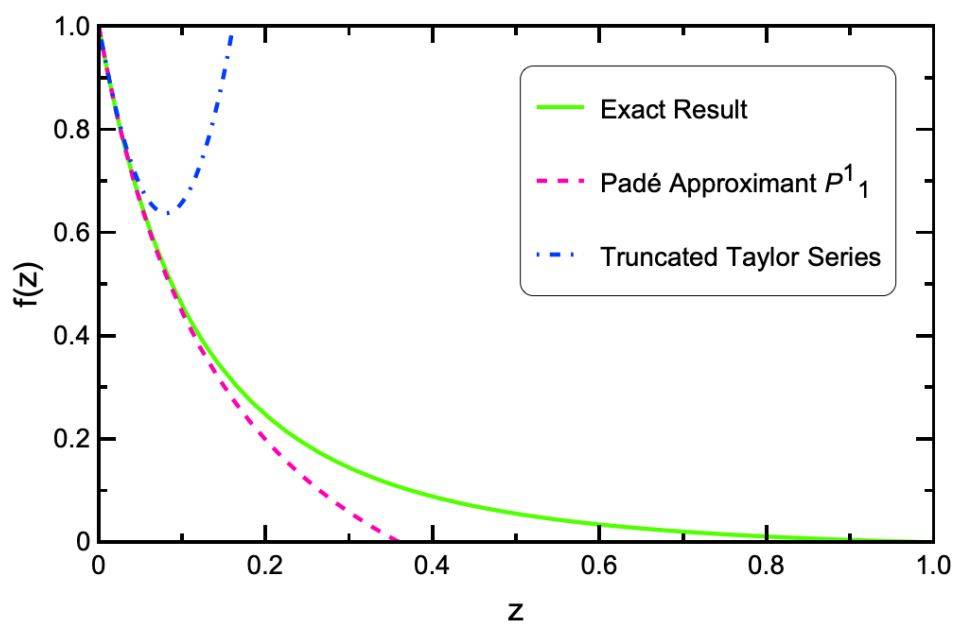

Figure 22 - Comparison between the exact result of $f(z)=\frac{1-z}{(1+4 z)^{2}}$, its Padé Approximant $P_{1}^{1}$ and its Taylor series truncated at second order.

Source: By the author.

$$
P_{1}^{1}(z)=\frac{1-\frac{25}{9} z}{1+\frac{56}{9} z} .
$$

Then, comparing the series expansion of $f(z)$ and $P_{1}^{1}$

$$
\begin{aligned}
f(z) & \approx 1-9 z+56 z^{2}-304 z^{3}+1536 z^{4}-7424 z^{5}+34816 z^{6}+\ldots, \\
P_{1}^{1}(z) & \approx 1-9 z+56 z^{2}-348 z^{3}+2168 z^{4}-13490 z^{5}+83940 z^{6}+\ldots,
\end{aligned}
$$

the predictions of the PA for the higher-order coefficients of the series can be read off. The estimates are written in magenta and we will always adopt this convention in this dissertation. The errors of the predicted third- and fourth-order coefficients are respectively $15 \%$ and $41 \%$, which can be considered as rather small since the PA only used up to the second-order coefficient of $f(z)$. The Padé gives also a prediction for the pole of the function $f(z)$. In this example, $f(z)$ has a double pole at $z=-0.25$, and $P_{1}^{1}$ has a single pole at $z=-0.16$, the pole position has an error of $36 \%$.

For meromorphic functions, in other words, whose singularities are just isolated poles, which is the case of $f(z)$, some of the poles and residues of the PA may be complex, even if the original function does not have complex poles. ${ }^{56}$ These poles cannot be identified with any singularity of the function, however it is still possible to employ the Padé to study the function away from them since close to their location the Padé approximation fails. These complex poles are transient, in other words, they appear and disappear when the order of the Padé is raised. ${ }^{56}$

In Fig. 22 it is possible to see how efficiently the Padé approaches the original function $f(z)$ compared to the truncated Taylor expansion. The green line represents $f(z)$, the blue one the Taylor series truncated at second order and in pink we have the Padé $P_{1}^{1}$. Using the same amount of coefficients as the Taylor series, the PA predicts the behavior of 
$f(z)$ more efficaciously.

In addition to using the standard Padés, we can employ other types of rational approximants, as the partial Padé approximant (PPA), where some knowledge about the analytical structure of the function is imposed, such as residues or poles. Considering that $k$ poles of the original function are imposed, then the PPA is defined as ${ }^{56}$

$$
\mathbb{P}_{N, k}^{M}(z)=\frac{Q_{M}(z)}{R_{N}(z) T_{k}(z)},
$$

where the polynomials $Q_{M}(z)$ and $R_{N}(z)$ are the same as before and $T_{k}(z)$ is constructed to have $k$ zeros exactly at the location of the lowest lying poles of the function $f(z)$

$$
T_{k}(z)=\left(z-p_{1}\right)^{m_{1}}\left(z-p_{2}\right)^{m_{2}} \ldots\left(z-p_{l}\right)^{m_{l}},
$$

with $p_{i}$ being the first $i$ pole of $f(z)$ and $\sum_{i=1}^{l} m_{i}=k$. As the PA, the PPA recreates precisely the first $M+N$ coefficients of the function $f(z)$ and its coefficients are determined in the same manner as for the PAs.

We are interested in Padé sequences where $M=N+k$, for $k$ being a fixed integer. For the case which $k \neq 0$, we have the near-diagonal sequence, and when $k=0$ the sequence is called diagonal. In the Padé approximant theory, there are some theorems that guarantee the convergence to the original function in a few cases. We will list two that apply to meromorphic functions.

Montessus' Theorem: Let $f(z)$ be a meromorphic function in the disk $|z| \leq R$, with $m$ poles in distinct positions $z_{1}, z_{2}, \ldots, z_{m}$ where

$$
0<\left|z_{1}\right| \leq\left|z_{2}\right| \leq \cdots \leq\left|z_{m}\right|<R
$$

Consider the pole $z_{k}$ with multiplicity $\mu_{k}$ and the total multiplicity precisely $\sum_{k=1}^{m} \mu_{k}=N$. Then

$$
\lim _{M \rightarrow \infty} P_{N}^{M}(z)=f(z),
$$

uniformly in any compact subset

$$
\mathscr{D}_{N}=\left\{z,|z| \leq R, z \neq z_{k}, k=1,2, \ldots, m\right\} .
$$

Hence, this theorem guarantees the Padé convergence inside the circle $\left|z_{m}\right|<R$. In addition, it also ensures that the poles and residues of the PA converge to the original values when $M \rightarrow \infty$. It is important to point out that in Montessus' theorem the Padé converges only if the polynomial in the denominator has $N$ coefficients in order to reproduce the analytical structure of the original function.

Pomerenke's Theorem: Let $f(z)$ be an analytic function at the origin and at the entire $z$ plane except for a countable number of isolated poles and essential singularities. 
Then the Padé sequence with $M=\lambda N$ (with $\lambda \neq 0$ and $\lambda \neq \infty$ ) satisfies

$$
\lim _{N \rightarrow 0} P_{N}^{\lambda N}(z)=f(z)
$$

in any compact set of the $z$ plane.

This theorem ensures that, in any compact region in the complex plane, the extraneous poles of the Padé will move far from this region when the order of the PA is increased or they will appear in pairs close to zeros of the function, constituting what is known as defects. A corollary of this theorem ${ }^{11}$ guarantees that it can be generalized to Padé sequences as $P_{N}^{N+k}$, for fixed $k$, and the convergence also occurs when $N \rightarrow \infty$.

The substantial advantage of using Pomerenke's theorem instead of Montessus' one is that is not necessary to previously know the number of poles of the original function. On the other hand, the disadvantage is that when the order of the Padé sequence $P_{N}^{N+k}$ is increased by one, it is necessary, at least, two new coefficients as input parameters (one for the numerator and another for the denominator of the PA), while in the Montessus' theorem case, raising one order in the Padé demands only one new parameter, since the denominator's order is fixed. In this work we will follow the spirit of Pomerenke's theorem.

As we will see, in the large- $\beta_{0}$ limit the Borel transform of the imaginary part of the scalar correlator is a meromorphic function. The Pomerenke's theorem will then be fundamental in this limit due to the appearance of the aforementioned defects in some cases, which will be analyzed in Sec. 5.2.

However, the Borel transform of the imaginary part in QCD contains cuts instead of poles. And there are no convergence theorems for functions with cuts, but we can analyze in practice if Padés are able to approximate functions of this type.

We will examine now the function $g(z)=\sqrt{\frac{3+z}{1+z}}$, that has a cut on the real axis along $[-3,-1]$. In order to construct the Padé $P_{1}^{1}$, we perform as before: expand $g(z)$ and $P_{1}^{1}$ and compare the coefficients of the same order. So the Padé $P_{1}^{1}$ is given by

$$
P_{1}^{1}(z)=\frac{1.732+0.866 z}{1+0.833 z}
$$

Expanding both $f(z)$ and $P_{1}^{1}$

$$
\begin{aligned}
g(z) & \approx 1.732-0.577 z+0.481 z^{2}-0.417 z^{3}+0.372 z^{4}-0.338 z^{5}+0.311 z^{6}+\ldots \\
P_{1}^{1}(z) & \approx 1.732-0.577 z+0.481 z^{2}-0.401 z^{3}+0.334 z^{4}-0.278 z^{5}+0.232 z^{6}+\ldots,
\end{aligned}
$$

one can notice that $P_{1}^{1}$ makes predictions for the coefficients starting from the third order. Theses predictions are excellent, with errors around $4 \%$ and $10 \%$ for third and fourth orders. Furthermore, the PA mimics the sign-alternating behavior of $g(z)$ and has a pole at $z=-1.2$, which is inside the cut region.

One can also analyze the convergence of the Padé studying the sequence $P_{N}^{N}$. For this 


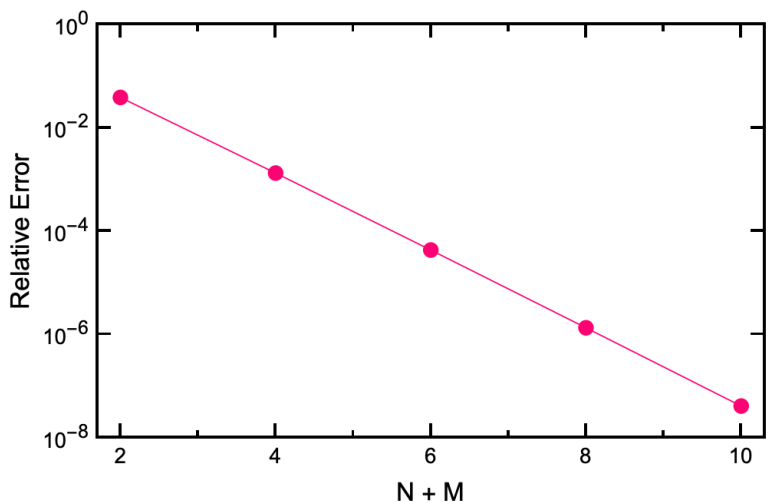

Figure 23 - Relative error of the first coefficient predicted by $P_{N}^{N}$ obtained from $g(z)=\sqrt{\frac{3+z}{1+z}}$.

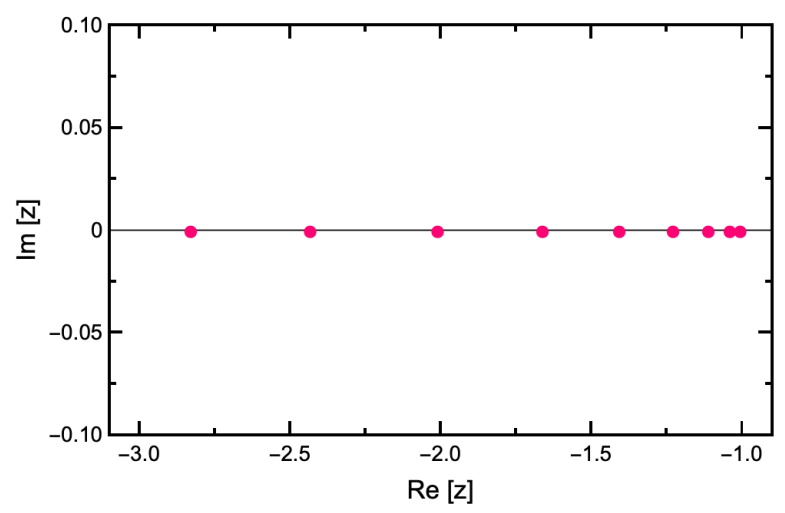

Figure 24 - Poles predicted by $P_{13}^{13}$ obtained from $g(z)=\sqrt{\frac{3+z}{1+z}}$.

Source: By the author.

purpose, the relative error of the first coefficient predicted by the PA will be examined and its definition is

$$
\sigma_{\mathrm{rel}}=\left|\frac{c_{n}^{P}-c_{n}}{c_{n}}\right|,
$$

where $c_{n}$ is the $n$ th-order coefficient of the original function and $c_{n}^{P}$ is the one predicted by the Padé. In Fig. 23 one can notice that when increasing the order of the Padé, the error reduces exponentially. Thus, apparently the PA converges to the exact result even though the function has a cut. Although there is no theorem, in practice the PA also approximates very well functions with cuts. ${ }^{18}$

It is interesting to investigate what happens in this case to the poles of the PA when the order of the Padé is raised. In Fig. 24 we have the location of the poles of $P_{13}^{13}$. One can observe that all of them lay inside the region of the cut $[-3,-1]$, then a PA which has a lot of poles "tries" to reproduce the cut through the accumulation of poles along the cut.

A different kind of Padé that is useful for functions with cuts or branch points is the D-log Padé. Before we define it, let us consider the following function

$$
f(z)=A(z) \frac{1}{(\mu-z)^{\gamma}}+B(z),
$$

where $A(z)$ and $B(z)$ are functions with little structure and analytic at $z=\mu$. We can define now a new function $F(z)$ near $z=\mu$ as $^{11,18}$

$$
F(z)=\frac{\mathrm{d}}{\mathrm{d} z} \ln f(z) \approx \frac{\gamma}{(\mu-z)} .
$$

Even though $\gamma$ is not an integer, i.e., the function $f(z)$ has a cut, $F(z)$ has a simple pole and the residue is the exponent of the cut of $f(z)$. Thus, with $P_{N}^{M}(z)$ being the Padé 
constructed to $F(z)$ defined above, the D-log Padé, $\operatorname{Dlog}_{N}^{M}(z)$, of $f(z)$ is given by the expression below ${ }^{11,18}$

$$
\operatorname{Dlog}_{N}^{M}(z)=f(0) \exp \left\{\int \mathrm{d} z P_{N}^{M}(z)\right\} .
$$

Because of the derivative in Eq. (4.35), the $f(0)$ term is lost and must be reintroduced in order to correctly normalize the D-log Padé. The $\operatorname{Dlog}_{N}^{M}$ then reproduces exactly the first $M+N+1$ coefficients of $f(z)$. The D-log Padé is no longer a rational approximant, however the function $F(z)$ is meromorphic and, because of that, it is easily approximated by the Padé $P_{N}^{M}$.

It is possible to determine from the Padé applied to $F(z)$ the position of the cut $z=\mu$ and its residue $-\gamma$, which is related to the multiplicity of the singularity $\mu$ of the function $f(z)$. Since no assumption about $\mu$ or $\gamma$ is made, their estimates are exclusively obtained from the series coefficients.

As an example of how to construct the D-log Padé, the $\operatorname{Dlog}_{1}^{1}$ of the function $f(z)=$ $\frac{1}{(1+4 z)^{2}}$ will be calculated. This is the same function approximated by the PA $P_{1}^{1}$ of Eq. (4.27). First of all, we have to calculate the function $F(z)$ as defined in Eq. (4.35)

$$
F(z)=-\frac{8}{1+4 z}-\frac{1}{1-z} \text {. }
$$

It is possible to notice that the new function $F(z)$ has two single poles, one at $z=1$ and the other at $z=-0.25$. Hence, the new function transforms the double pole of $f(z)$ into a single pole. Now one needs to calculate the Padé $P_{1}^{1}$ to $F(z)$ in the same way done before, which results in

$$
P_{1}^{1}(z)=-\frac{9+\frac{200}{31} z}{1+\frac{129}{31} z} .
$$

Using Eq. (4.36), the $\operatorname{Dlog}_{1}^{1}$ of $f(z)$ can be constructed by calculating the integral of $P_{1}^{1}$ and then exponentiating the result, which leads to

$$
\operatorname{Dlog}_{1}^{1}(z)=\frac{3.625 \mathrm{e}^{-1.55 z}}{(0.24+z)^{1.79}}
$$

One can note that this D-log Padé predicts a pole at $z=-0.24$ with multiplicity 1.79, which is in very good agreement with the values of $f(z)$, a double pole at $z=-0.25$. We can determine now the higher-order coefficients of $\operatorname{Dlog}_{1}^{1}$, so expanding the D-log Padé and $f(z)$ we have

$$
\begin{aligned}
f(z) & \approx 1-9 z+56 z^{2}-304 z^{3}+1536 z^{4}-7424 z^{5}+34816 z^{6}+\ldots, \\
\operatorname{Dlog}_{1}^{1}(z) & \approx 1-9 z+56 z^{2}-304 z^{3}+1542 z^{4}-7519 z^{5}+35694 z^{6}+\ldots .
\end{aligned}
$$

The errors of the first two predicted coefficients (fourth- and fifth-order) are $0.4 \%$ and $1.3 \%$ respectively, which is one order of magnitude smaller than the ones obtained from 
$P_{1}^{1}$ of Eq. (4.27) showing how efficient the D-log Padés can be.

We close this section with a discussion of the Stieltjes function. These functions play an important role in Padé theory although, as we will see, they are not central to our work. A function $f(z)$ is said to be Stieltjes if it can be written as a Stieltjes integral according to the expression ${ }^{11}$

$$
f(z)=\int_{0}^{\infty} \frac{\mathrm{d} \phi(u)}{1+z u},
$$

where $\phi(u)$ is a measure on $0 \leq u<\infty$ which has for all $m \geq 0$ finite and positive $\mu_{m}$ moments defined as

$$
\mu_{m}=\int_{0}^{\infty} u^{m} \mathrm{~d} \phi(u) .
$$

The formal expansion of $f(z)$, which may not be convergent for any $z$ (except $z=0$ ), is given by

$$
f(z)=\sum_{m=0}^{\infty} \mu_{m}(-z)^{m},
$$

and is called Stieltjes series if the coefficients $\mu_{m}$ are the moments of a measure $\phi(u)$ on $0 \leq u<\infty$. From Eq. (4.42), one finds

$$
f(z)=\sum_{m=0}^{\infty}(-z)^{m} \int_{0}^{\infty} u^{m} \mathrm{~d} \phi(u) .
$$

A necessary condition for $f(z)$ to be a Stieltjes function is that all the Hankel determinants $D(m, n)$, with $m \geq 0$ and $n \geq 0$, constructed with the $\mu_{m}$ momenta of the function must be strictly positive, ${ }^{11}$ i.e.

$$
D(m, n)=\left|\begin{array}{cccc}
\mu_{m} & \mu_{m+1} & \cdots & \mu_{m+n} \\
\mu_{m+1} & \mu_{m+2} & \cdots & \mu_{m+n+1} \\
\vdots & \vdots & \ddots & \vdots \\
\mu_{m+n} & \mu_{m+n+1} & \cdots & \mu_{m+2 n}
\end{array}\right|>0 .
$$

A Padé sequence of a Stieltjes function has some interesting convergence properties which may be useful. It is possible to show that for $z>0^{11}$

* the Padé sequences $P_{N}^{N-1}(z)$ increase monotonically when $N$ increases;

* the Padé sequences $P_{N}^{N+J}(z)$, with $J \geq-1$, uniformly converge to a continuous function $f^{(J)}(z)$;

* the poles of the Padés $P_{N}^{N+J}(z)$, with $J \geq-1$, are simple ones and lie on the negative real axis of $z$ with positive residues. 
If the function $f(z)$ is analytic at $|z|<R$, then the poles of the Padés $P_{N}^{N+J}(z)$, with $J \geq-1$, lie on the real axis in the interval $-\infty<z<-R$. Also, the function $f^{(J)}(z)$ the Padés converge to, is equal to the original function $f(z)$ for all $J \geq-1$. An important consequence of these properties is that the Padé sequences $P_{N}^{N-1}(z)$ and $P_{N}^{N}(z)$ act as a lower and upper bounds of $f(z)$, i.e.

$$
\lim _{N \rightarrow \infty} P_{N}^{N-1}(z) \geq f(z) \geq \lim _{N \rightarrow \infty} P_{N}^{N}(z)
$$

The above inequalities can be generalized in the following way: for any $J \geq-1$, the Padé sequence $P_{N}^{N+J}(z)$ generated from a Stieltjes function is monotonically increasing if $J$ is odd and monotonically decreasing if $J$ is even.

We will use the Padé approximants properties and convergence theorems in the large- $\beta_{0}$ limit, where all coefficients are known, to determine an efficient method to predict higherorder coefficients for the imaginary part of the scalar correlator in QCD. However, we will not be able to use the Stieltjes function's convergence theorems since all the functions we will study are not Stieltjes. 


\section{LARGE- $\beta_{0}$ LIMIT}

We will first apply the Padé approximants to the perturbative series in the limit called large- $\beta_{0} \cdot{ }^{19}$ We choose to work initially in this limit because this is an entirely consistent simplified model where higher-order corrections are known up to all orders in the coupling, thus the method applied can be evaluated quantitatively. The IR renormalons of the large- $\beta_{0}$ limit are connected with the operators present in the Operator Product Expansion (OPE) of the correlator. These renormalons, due to their location on the positive real axis, have an ambiguity associated, which can be, in principle, nullified by the non-perturbative contributions from OPE. However, just a part of these non-perturbative contributions can be calculated in practice, thus the cancellations in QCD cannot be demonstrated although it is expected to happen. ${ }^{9,10,57}$ An important fact is that the singularities in QCD are at the same position as the renormalons in large- $\beta_{0}$, however they are branch cuts, not poles. ${ }^{9}$

The contributions that are considered in this limit come from the insertion of fermion loops in the gluon propagator that appears at NLO as shown in Fig. 25. Those contributions are the same ones considered in Sec. 4.1 to the Adler function. In the large- $\beta_{0}$ limit, we need first to take the limit of large number of fermion flavors $N_{f}$, keeping the product $\alpha_{s} N_{f} \sim 1$ constant. Therefore, the corrections to the gluon propagator due to the insertion of $q \bar{q}$ bubble chains, whose diagrams are represented in Fig. 25, must be ressumed to all orders. The result in the large- $\beta_{0}$ limit is obtained by the non-abelianization procedure, discussed in Sec. 4.1, which consists of replacing $N_{f}$ with the dominant coefficient of the QCD $\beta$ function through the expression $N_{f} \rightarrow-3 \beta_{1}$, which, in practice, truncates the $\beta$ function at first order".

In order to evaluate the contributions from the fermion-bubble chains, the Borel transform of the hole chain will be computed. The effective gluon propagator after the insertion of an infinite number of fermion loops is, in the Landau gauge,

$$
D^{\mu \nu}=\frac{1}{k^{2}+i 0}\left(\frac{k^{\mu} k^{\nu}}{k^{2}}-g^{\mu \nu}\right) \frac{1}{1+\Pi\left(k^{2}\right)},
$$

where the function $\Pi\left(k^{2}\right)$ is the renormalized fermion loop and is given by ${ }^{34}$

$$
\Pi\left(k^{2}\right)=-\frac{N_{f}}{6} a_{s}\left(\mu^{2}\right)\left[\ln \left(-\frac{k^{2}}{\mu^{2}}\right)-\frac{5}{3}+C\right] .
$$

In the $\overline{\mathrm{MS}}$ scheme, where $\ln 4 \pi$ and $\gamma_{E}$ are also subtracted in the renormalization, the

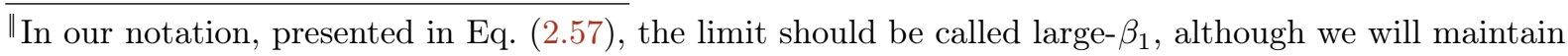
the nomenclature established in literature. 


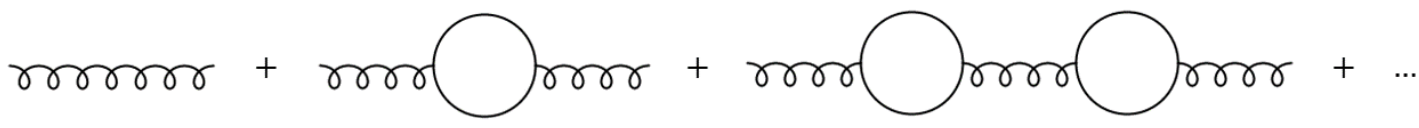

Figure 25 - Gluon propagator corrected by fermion-loop chains.

Source: By the author.

constant $C$ is zero**. We apply the naive non-abelianization, where $N_{f}$ is substituted by $-3 \beta_{1}$, and then the dressed gluon propagator, illustrated in Fig. 25, is ${ }^{9,34}$

$$
a_{s}\left(k^{2}\right) \delta_{a b} D^{\mu \nu}=i \delta_{a b}\left(\frac{k^{\mu} k^{\nu}-k^{2} g^{\mu \nu}}{k^{4}}\right) \frac{a_{s}\left(\mu^{2}\right)}{1+\frac{\beta_{1}}{2} a_{s}\left(\mu^{2}\right) \ln \left(-\frac{k^{2}}{\mu^{2}} \mathrm{e}^{C-5 / 3}\right)},
$$

where it was multiplied by the QCD coupling $a_{s}=\alpha_{s} / \pi$ at the scale $k^{2}$, the gluon momentum. Expanding the last fraction in series one obtains

$$
\frac{a_{s}\left(\mu^{2}\right)}{1+\frac{\beta_{1}}{2} a_{s}\left(\mu^{2}\right) \ln \left(-\frac{k^{2}}{\mu^{2}} \mathrm{e}^{C}\right)}=\sum_{n=0}^{\infty} a_{s}^{n+1}\left(-\frac{\beta_{1}}{2}\right)^{n} \ln ^{n}\left(-\frac{k^{2}}{\mu^{2}} \mathrm{e}^{C-5 / 3}\right) .
$$

In this way, the gluon propagator is now a series in $a_{s}$ where each order contains the highest power of $N_{f}$ from QCD. Performing now the Borel transform of the expression above, as indicated in Eq. (4.14), one gets

$$
\sum_{n=0}^{\infty} a_{s}^{n+1}\left(-\frac{\beta_{1}}{2}\right)^{n} \ln ^{n}\left(-\frac{k^{2}}{\mu^{2}} \mathrm{e}^{C-5 / 3}\right) \Longrightarrow\left(-\frac{\mu^{2} \mathrm{e}^{-C+5 / 3}}{k^{2}}\right)^{\beta_{1} t / 2},
$$

that generates the transformed propagator below ${ }^{9,34}$

$$
B\left[a_{s}\left(k^{2}\right) \delta_{a b} D^{\mu \nu}\right]=\frac{i \delta_{a b}}{k^{2}}\left(-g^{\mu \nu}+\frac{k^{\mu} k^{\nu}}{k^{2}}\right)\left(-\frac{\mu^{2} \mathrm{e}^{-C+5 / 3}}{k^{2}}\right)^{u},
$$

where we defined $u=\frac{\beta_{1} t}{2}$.

Hence, to determine the expression for a diagram that includes just one bubble chain it is necessary to compute the diagram with the gluon propagator given in Eq. (5.6). In practice, ${ }^{34}$ the term $-\frac{1}{k^{2}}$ that appears in the gluon propagator in the NLO diagrams should be replaced by $\left(-\frac{1}{k^{2}}\right)^{1+u}$.

\subsection{Scalar Correlator and Renormalons in the Large- $\boldsymbol{\beta}_{0}$ Limit}

In this dissertation we will apply the Padé approximants to the Borel transform of the perturbative series in the large- $\beta_{0}$ limit first since in this case we know the result to all orders. The objective is to determine the best method to apply in QCD, where we know just the first four coefficients. At this point we will analyze the scalar correlator in the large- $\beta_{0}$ limit and its renormalons.

\footnotetext{
${ }^{* *}$ Another convention used in some papers is to define $C^{\prime}=C-5 / 3$, thus in the $\overline{\mathrm{MS}}$ scheme $C^{\prime}=-5 / 3$.
} 


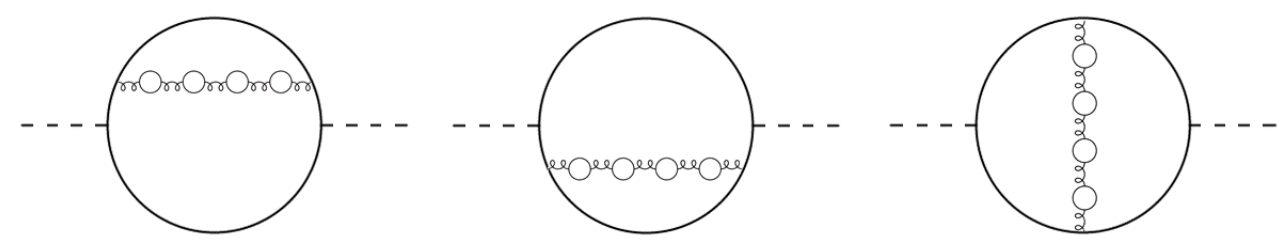

Figure 26 - Diagrams that contribute to the first-order correction in the large- $\beta_{0}$ limit.

Source: By the author.

The first-order correction to the scalar correlator in this limit is shown in Fig. 26 and, instead of the single gluon line we had before in QCD in Fig. 16, there is now a gluon propagator with a fermion-bubble chain. Thus, in order to determine this correction, we have to use the dressed gluon propagator, whose expression is in Eq. (5.6). Furthermore, the result to all orders, which is available in the 2001 paper of Broadhurst, Kataev and Maxwell, ${ }^{20}$ involves the quark mass renormalization in large- $\beta_{0}$, hence the $\gamma$ function will appear. This calculation follows the formalism described by Grozin ${ }^{58}$ and the results present here appear in a similar form to the ones reported by Jamin and Miravitllas. ${ }^{41}$

In the large- $N_{f}$ limit, the scalar correlator $\Pi_{L \beta}(s)$ is given by ${ }^{20}$

$$
\Pi_{L \beta}(s)=-\frac{N_{c}}{8 \pi^{2}} m^{2} s\left[L-2+\frac{C_{F} b}{2 T_{F} N_{f}} H(L, b)+\mathscr{O}\left(\frac{1}{N_{f}^{2}}\right)\right],
$$

where $L \equiv \ln \left(-\frac{s}{\mu^{2}}\right)$ and the strong coupling enters through the variable $b=\frac{T_{F} N_{f} a_{s}}{3}$. The function $H(L, b)$ can be expanded as

$$
H(L, b)=\sum_{n=1}^{\infty} H_{n+1}(L) b^{n-1}=\sum_{n=1}^{\infty} H_{n+1}(L)\left(\frac{T_{F} N_{f}}{3 \pi}\right)^{n-1} \alpha_{s}^{n-1},
$$

whose coefficients $H_{n}(L)$ are calculated through the formula below ${ }^{20}$

$$
n(n+1) H_{n+1}(L)=(n+1)\left[h_{n+2}+4(L-2) g_{n+1}\right]+4 g_{n+2}+9(-1)^{n} \mathscr{D}_{n+1}(L) .
$$

The parameters $g_{n}$ are the coefficients of the function $g(b)$, which is the contribution to all orders to the quark-mass anomalous dimension $\gamma_{m}(a)$ in the large- $N_{f}$ limit. In this limit the anomalous dimension is determined $b^{20}$

$$
\gamma_{m}(a)=-\frac{\mu}{m} \frac{\mathrm{d} m}{\mathrm{~d} \mu}=\frac{2 C_{F} b}{T_{F} N_{f}} g(b)+\mathscr{O}\left(\frac{1}{N_{f}^{2}}\right),
$$

with $C_{F}$ being the quadratic Casimir operator in the fundamental fermion representation and $T_{F}$ specifies the normalization of the corresponding matrices. In QCD, $C_{F}=4 / 3$ and $T_{F}=1 / 2$. One can obtain the coefficients $g_{n}$ through the following expression

$$
g(b)=\sum_{n=1}^{\infty} g_{n} b^{n-1}=\frac{(3-2 b)^{2}}{(4-2 b)} \frac{\Gamma(2-2 b)}{[\Gamma(2-2 b)]^{2}} \frac{\sin \pi b}{\pi b} .
$$

Thus, the expansion for the function $g(b)$, which is an efficient way to obtain its coefficients, 
is shown below ${ }^{20,41}$

$$
g(b)=\sum_{n=1}^{\infty} g_{n} b^{n-1}=\left[4-\sum_{n=2}^{\infty}\left(\frac{3}{2^{n}}+\frac{n}{2}\right) b^{n-2}\right] \exp \left(\sum_{k=3}^{\infty} \frac{2^{k}-3-(-1)^{k}}{k} \zeta(k) b^{k}\right) .
$$

Comparing the general expansions of $g(b)$ and $\gamma_{m}$, the relation between their coefficients in the large- $N_{f}$ limit is

$$
\gamma_{m}^{(n)}=4 C_{F} \frac{N_{f}^{n-1}}{6^{n}} g_{n}
$$

The coefficients $h_{n}$ that appear in Eq. (5.9) are scheme dependent constants and are related to the scalar-scalar anomalous dimension $\gamma_{S S} .{ }^{8,20}$ They can be computed through the following formula ${ }^{20}$

$$
\frac{h_{n+1}+4 g_{n+1}}{4}=\frac{4 g_{n-2}-4 g_{n-1}-5 g_{n}}{3 n} .
$$

The last piece in the result of Eq. (5.9) is the function $\mathscr{D}_{n}(L)$, which is determined by the following Borel transform

$$
\sum_{n=0}^{\infty} \frac{\mathscr{D}_{n}(L)}{n !} u^{n}=\left[1+u G_{D}(u)\right] \mathrm{e}^{-(L-5 / 3) u},
$$

where the term $5 / 3$ appears in the exponent since we are working in the $\overline{\mathrm{MS}}$ scheme and the function $G_{D}(u)$ is given by

$$
G_{D}(u)=\frac{2}{1-u}-\frac{1}{2-u}+\frac{2}{3} \sum_{k=3}^{\infty} \frac{(-1)^{k}}{(k-u)^{2}}-\frac{2}{3} \sum_{k=1}^{\infty} \frac{(-1)^{k}}{(k+u)^{2}} .
$$

The renormalons, which are present in the expression above, are the singularities of $G_{D}(u)$ and will be discussed in more detail later.

Applying the naive non-abelianization, which consists in changing $N_{f}$ for $-3 \beta_{1}$, the scalar correlator in the large- $\beta_{0}$ limit is obtained analytically up to second order as

$$
\begin{aligned}
\Pi_{L \beta}(s)=\frac{N_{c}}{4 \pi^{2}} m^{2} s\left[1-\frac{L}{2}-\frac{1}{9} \sum_{n=1}^{\infty}\left(-\frac{\beta_{1}}{2}\right)^{n-1} H_{n+1}(L) a_{s}^{n}\right]= \\
=\frac{N_{c}}{4 \pi^{2}} m^{2} s\left[1-\frac{L}{2}+a_{s}\left(\frac{131}{24}-\frac{17 L}{6}+\frac{L^{2}}{2}-2 \zeta_{3}\right)+a_{s}^{2}\left(\frac{11753}{432}-\frac{1495 L}{96}+\right.\right. \\
\left.\left.\quad+\frac{23 L}{6} \zeta_{3}+\frac{253 L^{2}}{72}-\frac{23 L^{3}}{72}+\frac{23}{36} \psi_{1}^{(2)}-\frac{115}{18} \zeta_{3}\right)+\ldots\right]
\end{aligned}
$$

where the quark mass and the QCD coupling are renormalized at the scale $\mu, \psi_{i}^{(2)}$ is the second derivative of the digamma function and we adopted $N_{f}=5$ to compute the second-order coefficient. The result at first order in $a_{s}$ should be equal in QCD and large- $\beta_{0}$ 
since it is independent of $N_{f}$. We can notice that the coefficient of $a_{s}$ shown above is equal to the one in Eq. (3.48) calculated by us in Sec. 3.2.3, after renormalization.

Choosing the scale $\mu$ as $\mu^{2}=-s$, so all the logarithms are resummed, the coefficients for the scalar correlator using $N_{f}=5$ are

$$
\Pi_{L \beta}(s)=\frac{N_{c}}{4 \pi^{2}} m^{2} s\left[1+3.0542 a_{s}+17.990 a_{s}^{2}+63.519 a_{s}^{3}+443.45 a_{s}^{4}+2958.45 a_{s}^{5}+\ldots\right] .
$$

This result can be compared with the one in QCD. In order to do that, we will write the coefficients in terms of $N_{f}$

$$
\begin{aligned}
\Pi_{L \beta}(s)=\frac{N_{c}}{4 \pi^{2}} m^{2} s\left[1+3.0542 a_{s}+\left(25.812-1.5644 N_{f}\right) a_{s}^{2}+\right. & \\
& \left.+\left(130.76-15.850 N_{f}+0.4803 N_{f}^{2}\right) a_{s}^{3}+\ldots\right] .
\end{aligned}
$$

Doing the same with the series of $\Pi(s)$ in QCD given by Eq. (3.50) for $\mu^{2}=-s$ we have

$$
\begin{aligned}
\Pi(s)=\frac{N_{c}}{4 \pi^{2}} m^{2} s\left[1+3.0542 a_{s}\right. & +\left(30.092-1.5644 N_{f}\right) a_{s}^{2}+ \\
& \left.+\left(353.32-36.822 N_{f}+0.4803 N_{f}^{2}\right) a_{s}^{3}+\ldots\right] .
\end{aligned}
$$

The result in large- $\beta_{0}$ reproduces exactly the highest-order term in $N_{f}$ for each power of $a_{s}$ of the scalar correlator series in QCD as expected. We can also compare the values of the coefficients. The values for $N_{f}=5$ in large- $\beta_{0}$ are in Eq. (5.18) and the ones for QCD are

$$
\Pi(s)=\frac{N_{c}}{4 \pi^{2}} m^{2} s\left[1+3.0542 a_{s}+22.2702 a_{s}^{2}+181.221 a_{s}^{3}+\ldots\right] .
$$

As we can notice, the large- $\beta_{0}$ limit is a good approximation in this case since the signs are all the same and the coefficient values are rather similar.

In order to isolate the non-trivial part of the perturbative series of the scalar correlator, we can define a function $J_{L \beta}$ as

$$
J_{L \beta}=\frac{4 \pi^{2}}{N_{c} m^{2} s} \Pi_{L \beta}(s)-1=-\frac{1}{9} \sum_{n=1}^{\infty}\left(-\frac{\beta_{1}}{2}\right)^{n-1} H_{n+1} a_{s}^{n} .
$$

Using Eq. (4.14), changing the variable $u=\frac{\beta_{1} t}{2}$ and employing the definitions for the coefficients $H_{n}$, the Borel transform of the function $J_{L \beta}$ can be written as

$$
\begin{aligned}
B\left[J_{L \beta}\right](u)= & -\frac{1}{9} \sum_{n=1}^{\infty} \frac{(-1)^{n-1}}{(n-1) !} H_{n+1} u^{n-1}=-\frac{1}{9} \sum_{n=1}^{\infty} \frac{(-1)^{n-1}}{(n-1) !} C_{n+1} u^{n-1}-\frac{1}{u^{2}}-\frac{11}{3 u}+ \\
& +\frac{\mathrm{e}^{5 u / 3}}{u}\left[\frac{1}{u}+\frac{2}{1-u}-\frac{1}{2-u}+\frac{2}{3} \sum_{k=3}^{\infty} \frac{(-1)^{k}}{(k-u)^{2}}-\frac{2}{3} \sum_{k=1}^{\infty} \frac{(-1)^{k}}{(k+u)^{2}}\right], \quad(5.21
\end{aligned}
$$

whose coefficients $C_{n}$ are defined as

$$
C_{n+1}=H_{n+1}-\frac{9(-1)^{n}}{n(n+1)} \mathscr{D}_{n+1} .
$$


This Borel transform does not appear in the papers of Broadhurst, Kataev and Maxwell ${ }^{20}$ or in the one of Jamin and Miravitllas, ${ }^{41}$ but it can be calculated from the results found in these references.

The terms of the first line in the Borel transform do not contain renormalons, because the possible renormalon at $u=0$ is canceled by the expression in the second line. Thus there are renormalons at $u=1,2,3, \ldots$ and $u=-1,-2,-3, \ldots$, where only the poles $u=1$ and $u=2$ are simple ones, the others are double poles. The closest poles to the origin are $u=1$ and $u=-1$, hence the residues have to be analyzed. Since the latter has a smaller residue, we expect that the renormalon $u=1$ governs the series behavior, which will have fixed-sign coefficients. This can be verified in Eq. (5.18), where all the series coefficients are positive.

The position of these renormalons does not change in full $\mathrm{QCD},{ }^{9}$ as already stated before. In addition, the location of the IR renormalons, $u=n$ for $n>0$, is related to the operators of dimension $2 n$ that participate in the Operator Product Expansion (OPE) of the scalar correlator, ${ }^{9,10}$ due to the uncertainty associated with the truncation of the asymptotic series. Since there is no gauge-invariant operator of dimension two in massless QCD, the renormalon $u=1$ of the scalar correlator is associated with the fact that this function is not a physical observable. It is expected that this renormalon disappears in physical quantities as we will see afterward. The IR pole at $u=2$ is related to the gluon condensate and is a physical renormalon in this sense. It introduces an ambiguity in the Borel integral, as we explained in Ch. 4, which can be canceled, at least in principle, by non-perturbative terms of the gluon condensate. The same happens for the other IR renormalons, $u=3,4, \ldots$, which would generate an exact series for the correlator, i.e., a series without ambiguities. ${ }^{9,10,57}$

Since the decay width is related to the imaginary part of the scalar correlator through the Optical Theorem, we will now calculate the imaginary part. Taking the imaginary part of Eq. (5.7) and choosing $\mu^{2}=s$ in the large- $N_{f}$ limit one finds ${ }^{20}$

$$
\operatorname{Im} \Pi_{L \beta}=\frac{N_{c}}{8 \pi} m^{2} s\left[1+\frac{3}{T_{F} N_{f}} \sum_{n=1}^{\infty} b^{n} S_{n+1}\right]=\frac{N_{c}}{8 \pi} m^{2} s\left[1+\sum_{n=1}^{\infty}\left(\frac{T_{F} N_{f}}{3}\right)^{n-1} S_{n+1} a_{s}^{n}\right],
$$

where the coefficients $S_{n}$ can be calculated through

$$
\begin{aligned}
S_{n+1} & =A_{n+1}+\Delta_{n+1}, \\
A_{n+1} & =\frac{(-1)^{n}}{n-1} \frac{g_{n}}{g_{1}}, \\
\sum_{n=2}^{\infty} \frac{u^{n-1} \Delta_{n}}{(n-2) !} & =2 \mathrm{e}^{5 u / 3}\left[1+u G_{D}(u)\right] \frac{\sin \pi u}{\pi u}-2,
\end{aligned}
$$

with $g_{n}$ and $G_{D}(u)$ provided in Eqs. (5.12) and (5.16), and the exponent $5 / 3$ in the expression for $\Delta_{n}$ being related to the $\overline{\mathrm{MS}}$ scheme. 
The naive non-abelianization can be applied then to get the imaginary part of the scalar correlator in the large- $\beta_{0}$ limit

$$
\begin{aligned}
\operatorname{Im} \Pi_{L \beta} & =\frac{N_{c}}{8 \pi} m^{2} s\left[1+\sum_{n=2}^{\infty}\left(\frac{\beta_{1}}{2}\right)^{n-2} a_{s}^{n-1} S_{n}\right]=\frac{N_{c}}{8 \pi} m^{2} s\left[1+5.6667 a_{s}+15.625 a_{s}^{2}+\right. \\
& \left.+34.249 a_{s}^{3}+31.117 a_{s}^{4}+37.969 a_{s}^{5}-1403.66 a_{s}^{6}+6129.94 a_{s}^{7}-129864 a_{s}^{8}+\ldots\right]
\end{aligned}
$$

whose values were computed adopting $N_{f}=5$. These coefficients must be compared to the exact ones in QCD given in Eq. (3.55) and one can see that this limit is a good approximation up to the order $a_{s}^{3}$. The sign of the coefficient of order $a_{s}^{4}$ is the opposite of large- $\beta_{0}$. This indicates that the dominant renormalon at these orders is different in QCD.

As we did for the scalar correlator, we will write the coefficients above in powers of $N_{f}$ to compare them with the ones from QCD. Thus we have in the large- $\beta_{0}$ limit

$$
\begin{aligned}
\operatorname{Im} \Pi_{L \beta}= & \frac{N_{c}}{8 \pi} m^{2} s\left[1+5.6667 a_{s}+\left(22.4177-1.3587 N_{f}\right) a_{s}^{2}+\left(70.5057-8.5461 N_{f}+\right.\right. \\
& \left.\left.+0.2590 N_{f}^{2}\right) a_{s}^{3}+\left(91.9082-16.7106 N_{f}+1.0128 N_{f}^{2}-0.0205 N_{f}^{3}\right) a_{s}^{4}+\ldots\right] .
\end{aligned}
$$

Rewriting also the series of $\operatorname{Im} \Pi$ in QCD, present in Eq. (3.54), we have

$$
\begin{aligned}
\operatorname{Im} \Pi= & \frac{N_{c}}{8 \pi} m^{2} s\left[1+5.6667 a_{s}+\left(35.94-1.3587 N_{f}\right) a_{s}^{2}+\left(164.14-25.771 N_{f}+\right.\right. \\
& \left.\left.+0.2590 N_{f}^{2}\right) a_{s}^{3}+\left(39.337-220.93 N_{f}+9.6848 N_{f}^{2}-0.0205 N_{f}^{3}\right) a_{s}^{4}+\ldots\right] .
\end{aligned}
$$

The large- $\beta_{0}$ result contains all the highest-order terms in $N_{f}$ for every power of $a_{s}$ that are in the QCD coefficients, as expected.

We will define a more convenient function in order to study the Borel transform of the imaginary part. This function has only the $\alpha_{s}$ corrections of the series of the imaginary part and is given by

$$
F_{L \beta}=\frac{8 \pi}{N_{c} m^{2} s} \operatorname{Im} \Pi_{L \beta}-1=\sum_{n=1}^{\infty}\left(\frac{\beta_{1}}{2}\right)^{n-1} S_{n+1} a_{s}^{n} .
$$

Computing the Borel transform of the function above, reminding that $u=\frac{\beta_{1} t}{2}$, and employing the definitions for the $S_{n}$ coefficients given in Eqs. (5.24) to (5.26), one finds

$$
\begin{aligned}
B\left[F_{L \beta}\right](u)=\sum_{n=1}^{\infty} \frac{S_{n+1}}{(n-1) !} u^{n-1} & =\sum_{n=1}^{\infty} \frac{A_{n+1}}{(n-1) !} u^{n-1}+2 \mathrm{e}^{5 u / 3}\left[\frac{1}{u}+\frac{2}{1-u}-\frac{1}{2-u}+\right. \\
& \left.+\frac{2}{3} \sum_{k=3}^{\infty} \frac{(-1)^{k}}{(k-u)^{2}}-\frac{2}{3} \sum_{k=1}^{\infty} \frac{(-1)^{k}}{(k+u)^{2}}\right] \frac{\sin \pi u}{\pi u}-\frac{2}{u} .
\end{aligned}
$$

This Borel transform, given explicitly for the first time here, can be worked out from the results present in the papers of Broadhurst, Kataev and Maxwell ${ }^{20}$ and of Jamin and 
Miravitllas. ${ }^{41}$ The first term of the Borel transform does not contain any renormalons, which are in the final part of the expression. Due to the zeros of the function $\frac{\sin \pi u}{\pi u}$, there are no renormalons in $u=1$ and $u=2$ and the other ones, that would be double poles, are now simple poles. It was expected that there would be no renormalon at $u=1$, because the imaginary part is a physical observable. Since the pole closest to the origin is $u=-1$, it is presumed that $F_{L \beta}$ is a sign-alternating series at higher orders, which indeed can be verified in Eq. (5.27).

As in QCD, the imaginary part of the scalar correlator can also be written in terms of the invariant mass $\widehat{m}$, whose relation with $m(\mu)$ is given by Eq. (2.63). In the large- $\beta_{0}$ limit, the $\beta$ function is truncated at first order and the $\gamma$ function coefficients are obtained through Eq. (5.13) with $N_{f} \rightarrow-3 \beta_{1}$. In this way, the scale-dependent mass $m(\mu)$ is

$$
m(\mu) \equiv \widehat{m}\left[\alpha_{s}(\mu)\right]^{\gamma_{m}^{(1)} / \beta_{1}} \exp \left\{\sum_{n=1}^{\infty}\left[\frac{\gamma_{m}^{(n+1)}}{\beta_{1}} \frac{a_{s}^{n}}{n}\right]\right\} .
$$

Then expanding the expression above and keeping only the highest terms in $N_{f}$ the expression for the imaginary part of the scalar correlator is

$$
\begin{aligned}
\operatorname{Im} \Pi_{L \beta}(s)=\frac{N_{c}}{8 \pi} \widehat{m}^{2} s\left(\alpha_{s}\right)^{2 \gamma_{m}^{(1)} / \beta_{1}}\{1+ & \frac{3 C_{F}}{\beta_{1}} \int_{0}^{\infty} \mathrm{d} u \mathrm{e}^{-2 u /\left(\beta_{1} a_{s}\right)} \times \\
\times & \left.\left.\times\left(1+u G_{D}(u)\right) \frac{\sin \pi u}{\pi u} \mathrm{e}^{5 u / 3}-1\right] \frac{1}{u}\right\}
\end{aligned}
$$

whose coefficients for $N_{f}=5$ in the $\overline{\mathrm{MS}}$ scheme are

$$
\begin{array}{r}
\operatorname{Im} \Pi_{L \beta}(s)=\frac{N_{c}}{8 \pi} \widehat{m}^{2} s\left(\alpha_{s}\right)^{2 \gamma_{m}^{(1)} / \beta_{1}}\left[1+7.3333 a_{s}+13.761 a_{s}^{2}+31.185 a_{s}^{3}+30.727 a_{s}^{4}+\right. \\
\left.+40.061 a_{s}^{5}-1402.21 a_{s}^{6}+6130.65 a_{s}^{7}-129865 a_{s}^{8}+\cdots\right] .
\end{array}
$$

These coefficients can be compared to the QCD ones, given in Eq. (3.56). Differently of what happened for the imaginary part in terms of the scale-dependent quark mass, the large- $\beta_{0}$ limit in this case is not a good approximation for orders higher than two.

Another related quantity considered in the literature, ${ }^{41}$ since it is a physical observable that can be used in quark-mass extractions, is the second derivative of the scalar correlator. Taking the second derivative of Eq. (5.7) and converting $N_{f}$ to $-3 \beta_{1}$ in large- $\beta_{0}$ one has

$$
\begin{aligned}
\Pi_{L \beta}^{\prime \prime}(s)=- & \frac{N_{c}}{8 \pi^{2}} \frac{m^{2}}{s}\left\{1-\frac{2}{\beta_{1}} \sum_{n=1}^{\infty} \frac{\gamma_{m}^{(n+1)}}{n} a_{s}^{n}+\right. \\
& \left.+\frac{3 C_{F}}{\beta_{1}} \int_{0}^{\infty} \mathrm{d} u \mathrm{e}^{-2 u /\left(\beta_{1} a_{s}\right)}\left[(1-u)\left[1+u G_{D}(u)\right] \mathrm{e}^{-(L-5 / 3) u}-1\right] \frac{1}{u}\right\} .
\end{aligned}
$$


The second derivative can also be written in terms of the invariant mass. Then using Eq. (5.30) and keeping terms up to the order $\beta_{1}^{-1}, \Pi_{L \beta}^{\prime \prime}$ results in

$$
\begin{aligned}
\Pi_{L \beta}^{\prime \prime}(s)=-\frac{N_{c}}{8 \pi^{2}} \frac{\widehat{m}^{2}}{s}\left(\alpha_{s}\right)^{2 \gamma_{m}^{(1)} / \beta_{1}}\{1 & +\frac{3 C_{F}}{\beta_{1}} \int_{0}^{\infty} \mathrm{d} u \mathrm{e}^{-2 u /\left(\beta_{1} a_{s}\right)} \times \\
\times & \left.\left.\times(1-u)\left[1+u G_{D}(u)\right] \mathrm{e}^{-(L-5 / 3) u}-1\right] \frac{1}{u}\right\} .
\end{aligned}
$$

The coefficients for $N_{f}=5$ are

$$
\begin{array}{r}
\Pi_{L \beta}^{\prime \prime}(s)=-\frac{N_{c}}{8 \pi^{2}} \frac{\widehat{m}^{2}}{s}\left(\alpha_{s}\right)^{2 \gamma_{m}^{(1)} / \beta_{1}}\left[1+5.3333 a_{s}+6.0111 a_{s}^{2}+42.8911 a_{s}^{3}+0.7729 a_{s}^{4}+\right. \\
\left.+1512.48 a_{s}^{5}-8410.57 a_{s}^{6}+163609 a_{s}^{7}-2.09 \times 10^{6} a_{s}^{8}+\ldots\right],
\end{array}
$$

which have to be compared with the ones in Eq. (3.60), the exact values in QCD. We can observe that the coefficients have the same sign in QCD, however the values are considerably smaller in the large- $\beta_{0}$ limit, especially for the order $a_{s}^{4}$.

\subsubsection{Scheme Variation}

As we discussed in Sec. 2.3, it is possible to change the renormalization scheme of the strong coupling $a_{s}$ to a more perturbative scheme or a less perturbative one. This modification can lead to a greater or a minor dominance of the UV renormalons, which can accelerate the convergence of the Padé approximants that we will apply. The relation between the coupling in a scheme parametrized by a constant $C$ and one in the $\overline{\mathrm{MS}}$ in QCD is determined in Eq. (2.72). In the large- $\beta_{0}$ limit the $\beta$ function is truncated at first order thus this relation can be simplified to

$$
\frac{1}{\tilde{a}_{s}} \equiv \frac{1}{a_{s}^{\prime}}=\frac{1}{a_{s}^{\overline{\mathrm{MS}}}}+C \frac{\beta_{1}}{2} .
$$

Hence, to modify the scheme of a perturbative series, it is sufficient to substitute the coupling $a_{s}$ in the $\overline{\mathrm{MS}}$ scheme for the coupling $\tilde{a}_{s}$ parametrized by a constant $C$ using Eq. (5.36). Thus for $C=0$ we have the $\overline{\mathrm{MS}}$ scheme; $C$ positive corresponds to more perturbative cases, since the coupling decreases; and $C$ negative increases the value of the coupling, resulting in a less perturbative case.

One can change the renormalization scheme of the imaginary part of the scalar correlator in the large- $\beta_{0}$ limit, given by Eq. (5.31). The resulting expression is

$$
\begin{aligned}
\operatorname{Im} \Pi_{L \beta}(s)=\frac{N_{c}}{8 \pi} \widehat{m}^{2} s\left(\tilde{\alpha}_{s}\right)^{2 \gamma_{m}^{(1)} / \beta_{1}}\{1 & +\frac{3 C_{F}}{\beta_{1}} \int_{0}^{\infty} \mathrm{d} u \mathrm{e}^{-2 u /\left(\beta_{1} \tilde{a}_{s}\right)} \times \\
\times & {\left.\left[\left(1+u G_{D}(u)\right) \frac{\sin \pi u}{\pi u} \mathrm{e}^{(C+5 / 3) u}-1\right] \frac{1}{u}\right\} . }
\end{aligned}
$$


For $C=0$, we recover the previous result of Eq. (5.31) for the imaginary part. Hence, we are generalizing the expression for any $\overline{\mathrm{MS}}$-like scheme, since $C$ is a continuous parameter that allows the variations between infinite schemes where $C=0$ is the $\overline{\mathrm{MS}}$.

As we did before, we define the function $\widehat{F}_{L \beta}^{C}$, which we will call reduced imaginary part, to contain only the $\alpha_{s}$ corrections of the function above

$$
\begin{aligned}
\widehat{F}_{L \beta}^{C} & =\left(\tilde{\alpha}_{s}\right)^{-2 \gamma_{m}^{(1)} / \beta_{1}} \frac{8 \pi}{N_{c} \widehat{m}^{2} s} \operatorname{Im} \Pi_{L \beta}(s)-1= \\
& =\frac{3 C_{F}}{\beta_{1}} \int_{0}^{\infty} \mathrm{d} u \mathrm{e}^{-2 u /\left(\beta_{1} \tilde{a}_{s}\right)}\left\{\left[1+u G_{D}(u)\right] \frac{\sin \pi u}{\pi u} \mathrm{e}^{(C+5 / 3) u}-1\right\} \frac{1}{u} .
\end{aligned}
$$

Its Borel transform is given by

$$
\begin{aligned}
B\left[\widehat{F}_{L \beta}^{C}\right](u) & =\frac{3 C_{F}}{\beta_{1}}\left\{\left[1+u G_{D}(u)\right] \frac{\sin \pi u}{\pi u} \mathrm{e}^{(C+5 / 3) u}-1\right\} \frac{1}{u}=\frac{3 C_{F}}{\beta_{1}}\left\{\mathrm{e}^{(C+5 / 3) u} \frac{\sin \pi u}{\pi u} \times\right. \\
& \left.\times\left[\frac{1}{u}+\frac{2}{1-u}-\frac{1}{2-u}+\frac{2}{3} \sum_{k=3}^{\infty} \frac{(-1)^{k}}{(k-u)^{2}}-\frac{2}{3} \sum_{k=1}^{\infty} \frac{(-1)^{k}}{(k+u)^{2}}\right]-\frac{1}{u}\right\} .
\end{aligned}
$$

This Borel transform, as the one of Eq. (5.29), can be determined from the results present in Broadhurst, Kataev and Maxwell's paper ${ }^{20}$ and in the one of Jamin and Miravitllas. ${ }^{41}$ It is important to notice that the location of the renormalons of the Borel transform above does not change for different values of $C$, only their residues. The position of the renormalons are the same as in the Borel transform defined in Eq. (5.29). For negative values of $C$, the Borel transform is more dominated by the first UV pole, $u=-1$, due to the exponential term. Therefore, the sign-alternating behavior must be manifest in lower orders.

Analogously, the renormalization scheme can also be changed in the perturbative series of the second derivative. Employing Eq. (5.36) in Eq. (5.34) culminates in the expression ${ }^{41}$

$$
\begin{aligned}
\Pi_{L \beta}^{\prime \prime}(s)=-\frac{N_{c}}{8 \pi^{2}} \frac{\widehat{m}^{2}}{s}( & \left.\alpha_{s}^{C_{m}}\right)^{2 \gamma_{m}^{(1)} / \beta_{1}}\left\{1-\frac{2 \gamma_{m}^{(1)}}{\beta_{1}} \ln \left[1-\left(C_{m}+\frac{5}{3}\right) \frac{\beta_{1}}{2} a_{s}^{C_{m}}\right]+\right. \\
& \left.+\frac{3 C_{F}}{\beta_{1}} \int_{0}^{\infty} \mathrm{d} u \mathrm{e}^{-2 u /\left(\beta_{1} a_{s}^{C_{a}}\right)} \mathrm{e}^{\left(C_{a}+5 / 3\right) u}\left[(1-u) G_{D}(u)-1\right]\right\},
\end{aligned}
$$

where we introduced two separate constants $C_{m}$ and $C_{a}$ that refer to the dependence on the renormalization scheme of the quark mass and the QCD coupling respectively. The notation $\tilde{a}_{s}=a_{s}^{C}$ was used to make this difference explicit. We will consider $C_{m}=C_{a}=C$ in this dissertation.

Finally, one can define again a new function that facilitates the analysis of the Borel transform of the second derivative. The function $\widehat{D}_{L \beta}^{C}$, which we will refer as the reduced 
second derivative, is given by

$$
\widehat{D}_{L \beta}^{C}=-\left(\tilde{\alpha}_{s}\right)^{-2 \gamma_{m}^{(1)} / \beta_{1}} \frac{8 \pi^{2} s}{N_{c} \widehat{m}^{2}} \Pi_{L \beta}^{\prime \prime}(s)-1 .
$$

Adopting the same method employed before, the Borel transform of $\widehat{D}_{L \beta}^{C}$ is now

$$
\begin{array}{r}
B\left[\widehat{D}_{L \beta}^{C}\right](u)=\frac{3 C_{F}}{\beta_{1}}\left\{\left[(1-u)\left[1+u G_{D}(u)\right] \mathrm{e}^{(C+5 / 3) u}-1\right] \frac{1}{u}\right\}=\frac{3 C_{F}}{\beta_{1}}\left\{-\frac{1}{u}+\right. \\
\left.+\mathrm{e}^{(C+5 / 3) u}\left[\frac{1}{u}+\frac{1}{2-u}-\frac{2}{3} \sum_{k=3}^{\infty}(-1)^{k} \frac{(u-1)}{(k-u)^{2}}+\frac{2}{3} \sum_{k=1}^{\infty}(-1)^{k} \frac{(u-1)}{(k+u)^{2}}\right]\right\} .
\end{array}
$$

This Borel transform has only one simple pole, $u=2$. The other renormalons, $u=-1,-2,-3, \ldots$ and $u=3,4,5, \ldots$, are all double poles. Since the second derivative is physical, there is no renormalon at $u=1$. The closest one to the origin is $u=-1$, then it will govern the behavior of $\widehat{D}_{L \beta}^{C}$ at higher orders. Thus this function will be a sign-alternating series, which in the $\overline{\mathrm{MS}}$ scheme can be verified in Eq. (5.35). As we discussed for the Borel transform of $\widehat{F}_{L \beta}^{C}, B\left[\widehat{D}_{L \beta}^{C}\right]$ is more dominated by the UV pole $u=-1$ for negative values of $C$, hence the sign-alternation should start earlier (in a lower order coefficient compared to the $\overline{\mathrm{MS}}$ ). Furthermore, the location of the renormalons is not altered when the value of $C$ is modified, only the residues change.

Now that we have all the Borel transforms and the series in $\alpha_{s}$ in the large- $\beta_{0}$ limit, we can apply the Padé approximants in order to determine the best strategy to employ in the QCD case, where only the first four coefficients are known.

Analyzing all the Borel transforms and their renormalons, we expect that the standard Padé approximants will have difficulties to approximate the scalar correlator and its second derivative due to the double poles that force the Padés to spend coefficients "trying" to reproduce them. On the one hand, the function $\frac{\sin \pi u}{\pi u}$ turns the double poles into simple ones, which is an advantage for the imaginary part, on the other it may be complicated for the Padés to replicate the characteristics of this function. The presence of double poles is not a problem for the D-log Padés, since, as we saw in Sec. 4.3, they are transformed into single poles, thus we believe that the D-log Padés should work better than the standard Padés. Changing renormalization scheme to less perturbative ones, where the UV renormalons dominate more, is promising as well, since it will be easier for the Padé to replicate the sign-alternating behavior of the imaginary part and the second derivative. The particular scheme where $C=-5 / 3$ also has the benefit of canceling the exponential term for both cases, which may improve the Padé's results as we will see. 


\subsection{Padé Approximants to the Imaginary Part in the Large- $\boldsymbol{\beta}_{0}$}

In this section we will build Padé approximants to several Borel transforms discussed in the last section. Our main objective is to study the quality of the predictions of these Padés, in order to refine the method that will be applied in QCD.

We will start calculating Padés to the Borel transform of $F_{L \beta}$ in the large- $\beta_{0}$ limit, whose perturbative coefficients are in Eq. (5.27). This series contains only the non-trivial terms of the imaginary part written in terms of the scale-dependent quark mass $m(\mu)$. The dominant poles of this function are located at $u=-1$ and $u=-2$, which are simple poles. In addition, the sign-alternating behavior establishes at the sixth-order coefficient, indicating the dominance of the UV renormalon at intermediate and higher orders.

In the spirit of Pomerenke's theorem, we will work mostly with Padé sequences of the type $P_{N}^{N+k}$ with $k$ being an integer value. It is important to remind that a Padé $P_{N}^{M}$ applied to the Borel transform needs the first $M+N+1$ coefficients of the series in $\alpha_{s}$ and hence predicts the coefficient of order $M+N+2$.

Let us start with the analysis of the Padé sequence $P_{N}^{N}$. The first non-trivial Padé is $P_{1}^{1}$, which requires three coefficients as input and whose expression is

$$
P_{1}^{1}(u)=\frac{9.9096+8.5891 u}{1.7487-u} .
$$

The value of the first predicted coefficient, $c_{4}$, is 112.6, which is not a good prediction because the real value is 31.12 , as indicated in Eq. (5.27). This Padé also does not reproduce the sign-alternating behavior of $F_{L \beta}$ and its pole is located at $u=1.75$, that is not related to any renormalon of the Borel transform. This occurs due to the fact that in a function with many poles, the PA with less parameters predicts better the poles closer to the origin or it can generate effective poles, that do not correspond to any renormalon, just to reproduce the global behavior of the original series. ${ }^{11,12}$ Thus, it is expected that the pole of this PA would not obviously be related to any renormalon of the original function.

One can now analyze the next Padé in this sequence, $P_{2}^{2}$, which is evaluated as

$$
P_{2}^{2}(u)=\frac{22562.4+28902.5 u+13440.4 u^{2}}{(633.654+u)(6.28355-u)} .
$$

The poles of this PA cannot be identified with any renormalon of the original function and the coefficients are not sign-alternating, as the previous Padé. In addition, the prediction of the coefficient $c_{6}, c_{6}^{P}=57.908$, is not satisfactory as well, since the original one is -1403.6. A reason for the terrible results is the location of the poles: they are far from the origin, which, in practice, reduces the order of the Padé. This happens because $P_{2}^{2}$ can be split in a sum of the two poles and since the one at $u=-634$ is very distant from the origin, it does not contribute, effectively decreasing the order of the PA.

Let us consider the next Padé in this sequence, $P_{3}^{3}$. This Padé estimates the coefficient 


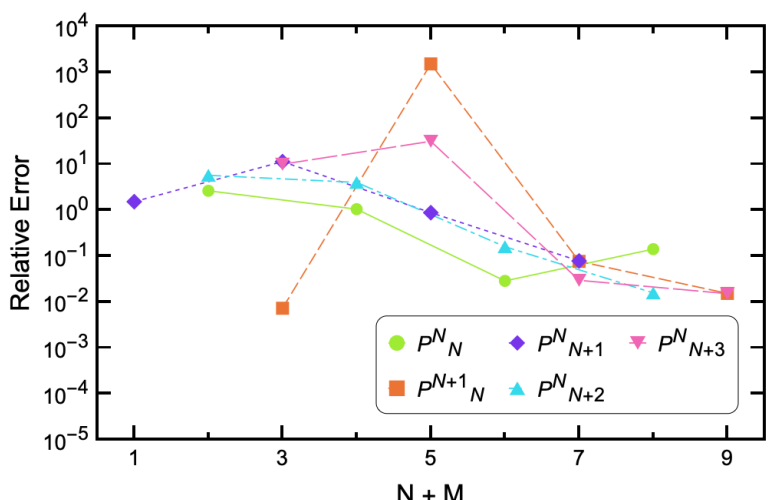

Figure 27 - Relative error for the first predicted coefficient $c_{n}$ for each Padé to the imaginary part.

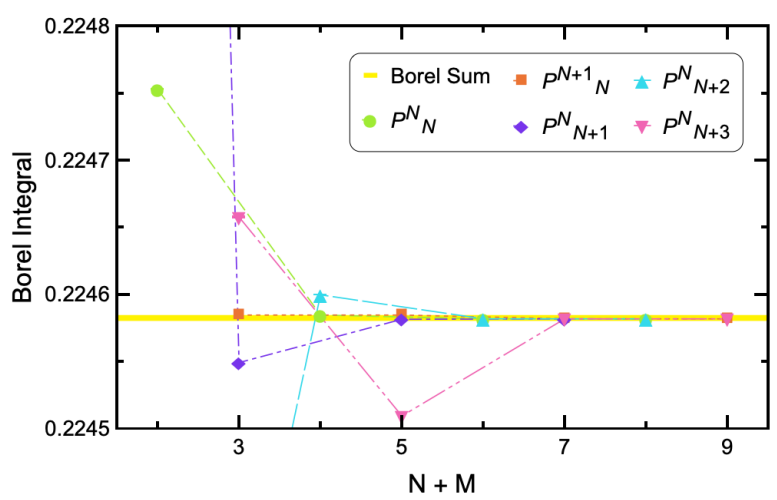

Figure 28 - Borel integral of $F_{L \beta}$ for several Padés to the imaginary part in large- $\beta_{0}$ using $\alpha_{s}\left(m_{H}\right)=0.1125$.

Source: By the author.

of order $a_{s}^{8}: c_{8}^{P}=-126164$, which represents an error of just $2.85 \%$. We also notice that when the order of the Padé is increased, the PA starts to mimic the renormalon closest to the origin, $u=-1$, as well as its residue. $P_{3}^{3}$ has a pole at $u=-0.9964$ with a residue of 0.2453 , where the pole position has an error of just $0.36 \%$ and the residue is $2.58 \%$ off the original value. As we can see, the Padé approximant works very well; this example shows how efficiently the PA reproduces the analytical structure of the original function and also indicates a convergence to the real values. However, the price to pay for excellent results is high: we need three more coefficients than we have in QCD. Thus it is important to search for other methods that also give good results but using less coefficients as input.

Other Padé sequences were investigated: $P_{N}^{N+1}, P_{N+1}^{N}, P_{N+2}^{N}$ and $P_{N+3}^{N}$. In order to verify the convergence of the sequences, we will analyze the relative error, defined in Eq. (4.33) of the first unknown coefficient in each case. The plot in Fig. 27 shows the relative error of the first predicted coefficient for all the five Padé sequences studied as a function of $N+M$. We can see that the error decreases when the order of the Padé is raised in most of the cases and the results are unsatisfactory for lower orders, as occurred for the sequence $P_{N}^{N}$. An increase by one unit in $N$ usually lowers the error by one order of magnitude. The cases where the error grows or does not reduce significantly can be explained by Pomerenke's theorem. This theorem states that the Padés can have what is called defects, poles almost canceled by a close-by zero, which effectively reduces the order of the PA. One can see this for example in the sequence $P_{N}^{N+1}$ shown in orange in Fig. 27: $P_{1}^{2}$ has a pole at $u=6.329$ and $P_{2}^{3}$ also has one pole at $u=6.329$ and another closer to the origin at $u=-0.0018$. This last pole, which could ruin the convergence of the PA, is almost exactly canceled by a zero at $u=-0.0018$, reducing the order of the Padé to a $P_{1}^{2}$. This cancellation is of the order of $10^{-17}$, in other words, the residue of the pole $u=-0.0018$ is $\mathscr{O}\left(10^{-17}\right)$. A similar behavior can also be observed in the Pade $P_{4}^{4}$, 
which appears in the right-most point in green in Fig. 27. When this almost cancellation happens we know that the result tends to be bad and therefore we can discard the Padé.

Furthermore, the PAs can be used to resum the original series through the calculation of the Borel integral using the reconstructed Padés. Fig. 28 exhibits the value of the integral in terms of $N+M$ for all the Padé sequences studied so far. We can observe that for $N+M>3$ most of the results are in good agreement with the one calculated from $F_{L \beta}$ and there is an apparent convergence to the true value. The ambiguity of the Borel integral is extremely small due to the low value of the QCD coupling at the Higgs mass, $\alpha_{s}\left(m_{H}\right)=0.1125$, which explains why it can barely be seen in the plot of Fig. 28 .

It was possible to observe that some Padés present extraneous poles, a pair of complex poles, which are not related to any singularity of the original function. As we explained in Ch. 4 , the appearance of these poles can happen when the function to be approximated is meromorphic ${ }^{11}$ and it does not prevent us to use the Padés to study the function away from these singularities.

Let us now turn to $\widehat{F}_{L \beta}^{C}$, the series of the non-trivial terms of the imaginary part of the scalar correlator written in terms of the invariant mass $\widehat{m}$. The Padé approximants were applied to the Borel transform of this function, given in Eq. (5.39), and the results found are essentially the same as the ones exhibited above, so we will abstain from showing them.

Considering that in QCD only the first four coefficients are known, it is necessary to analyze other strategies to obtain low errors with a smaller number of coefficients used as input, since the relative errors showed in Fig. 27 are only negligible for high values of $N$.

\subsubsection{Scheme Variation}

One strategy that we will consider at this point is changing the renormalization scheme. For the series of the reduced imaginary part written as a function of the invariant quark mass, $\widehat{F}_{L \beta}^{C}$, it is enough to alter the value of $C$. We choose to employ $C=-5 / 3$ because this is the value that exactly cancels the exponential in Eq. (5.38), which is an advantage since the PAs no longer have to deal with it. In addition, as already discussed, with negative values of $C$ the Borel transform is more dominated by the first UV pole, thus it is easier for the Padé to approximate this series. In this new scheme, the coupling is $\tilde{\alpha}_{s}\left(m_{H}\right)=0.1270$ and the coefficients of $\widehat{F}_{L \beta}^{(C=-5 / 3)}$ in the large- $\beta_{0}$ limit are

$$
\widehat{F}_{L \beta}^{(C=-5 / 3)}=4 \tilde{a}_{s}-4.3408 \tilde{a}_{s}^{2}+6.7614 \tilde{a}_{s}^{3}-58.7378 \tilde{a}_{s}^{4}+456.673 \tilde{a}_{s}^{5}-4161.77 \tilde{a}_{s}^{6}+\cdots .
$$

The sign-alternating behavior is already evident at lower orders, starting in the secondorder coefficient due to the residues of the poles. It exhibits the greater dominance of the renormalon $u=-1$ in schemes with a negative $C$. Without the exponential and with the higher dominance of the UV renormalon, we expect an improvement in the predictions.

We will now study the Padé approximants to the Borel transform of $\widehat{F}_{L \beta}^{(C=-5 / 3)}$ given 


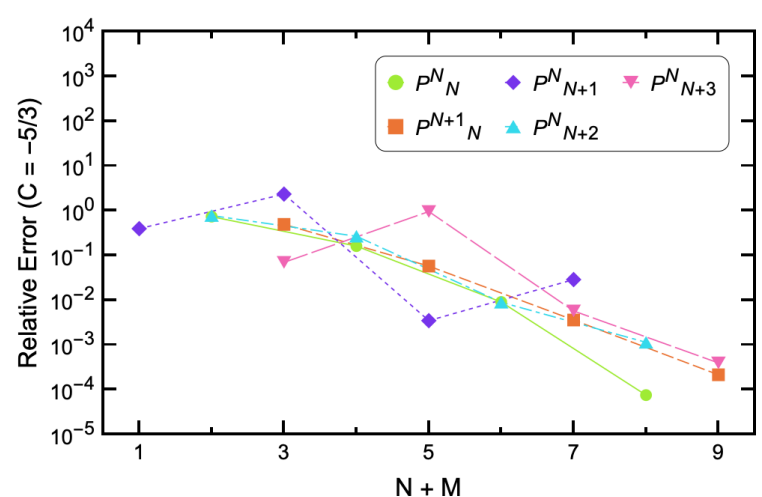

Figure 29 - Relative error for the first predicted coefficient $\widehat{c}_{n}$ for each Padé to the imaginary part using $C=-5 / 3$.

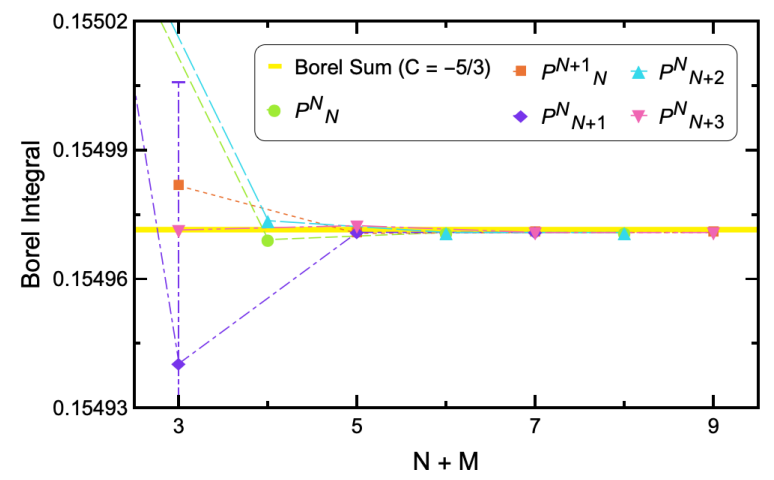

Figure 30 - Borel integral of $\widehat{F}_{L \beta}^{(C=-5 / 3)}$ for PAs to the imaginary part in large- $\beta_{0}$ using $C=-5 / 3$ and $\tilde{\alpha}_{s}\left(m_{H}\right)=0.1270$.

Source: By the author.

in Eq. (5.39) with $C=-5 / 3$. Let us consider again the Padé $P_{1}^{1}$ in the current scheme, whose expression is

$$
P_{1}^{1}(u)=\frac{-5.1360+0.8210 u}{2.4610+u} .
$$

The value for the first predicted coefficient $\widehat{c}_{4}$, is -15.7977 , with a relative error of 0.73 , one order of magnitude smaller than the one found for the same PA in the $\overline{\mathrm{MS}}$ scheme. One can see that the pole of this PA is located at $u=-2.461$, close to the poles $u=-2$ and $u=-3$ of the Borel transform, however those are not the dominant renormalons. Besides that, the PA also reproduces the sign-alternation of $\widehat{F}_{L \beta}^{(C=-5 / 3)}$, which did not occur for $P_{1}^{1}$ in the $\overline{\mathrm{MS}}$ scheme neither for $F_{L \beta}$ or $\widehat{F}_{L \beta}^{(C=0)}$.

Analyzing the next PA of the sequence $P_{N}^{N}$, we have

$$
P_{2}^{2}(u)=\frac{7.5588+2.0044 u-3.9059 u^{2}}{(u+0.9211)(3.9322-u)} .
$$

The first predicted coefficient is $\widehat{c}_{6}^{P}=-4830$, whose relative error is 0.16 , which is also one order lower than the one calculated in the $\overline{\mathrm{MS}}$ scheme. One can still note that this Padé already mimics the dominant pole $u=-1$ and its residue, with an error of $29 \%$. In addition, the sign-alternating behavior is correctly reproduced.

Other Padé sequences were also studied: $P_{N}^{N+1}, P_{N+1}^{N}, P_{N+2}^{N}$ and $P_{N+3}^{N}$ and the results for the relative error of the first predicted coefficient are shown in Fig. 29, which is in the same scale as the plot in Fig. 27 to facilitate the comparison. In general, the errors in the new scheme are approximately one order of magnitude smaller than the ones from the $\overline{\mathrm{MS}}$ scheme and the convergence to the real value happens faster as $N$ grows. For the sequences $P_{N+1}^{N}$ and $P_{N+3}^{N}$, that are represented respectively in purple and pink in Fig. 29, the appearance of the defects, that in practice reduces the order of the PA, causes the 
Table 1 - Coefficients of $F_{L \beta}$ (written in terms of the scale-dependent mass) in the $\overline{\mathrm{MS}}$. The values in blue are the exact ones in the large- $\beta_{0}$ limit. The Padés in white were applied to the Borel transform of $F_{L \beta}$ and the ones in pink to the Borel transform of $\widehat{F}_{L \beta}^{C}$. The terms inside the brackets indicate the scheme where the Padés were constructed.

\begin{tabular}{cccccc}
\hline & $c_{4}$ & $c_{5}$ & $c_{6}$ & $c_{7}$ & $c_{8}$ \\
\hline Large- $\beta_{0}($ exact $)$ & 31.117 & 37.969 & -1404 & 6130 & -129864 \\
$P_{1}^{1}(\overline{\mathrm{MS}})$ & 112.61 & 493.70 & 2706 & 17792 & 136501 \\
$P_{1}^{1}(\overline{\mathrm{MS}})$ & 106.39 & 478.35 & 2720 & 18505 & 146773 \\
$P_{1}^{1}(C=-5 / 3)$ & 74.057 & 179.19 & 440.26 & 1110 & 2584 \\
$P_{1}^{2}(\overline{\mathrm{MS}})$ & - & 37.695 & 57.078 & 103.72 & 219.87 \\
$P_{1}^{2}(\overline{\mathrm{MS}})$ & - & 38.277 & 64.851 & 130.93 & 301.10 \\
$P_{1}^{2}(C=-5 / 3)$ & - & 261.66 & -3520 & 54846 & $-1.16 \times 10^{6}$ \\
$P_{2}^{2}(\overline{\mathrm{MS}})$ & - & - & 57.908 & 105.98 & 226.29 \\
$P_{2}^{2}(\overline{\mathrm{MS}})$ & - & - & 63.838 & 127.94 & 291.97 \\
$P_{2}^{2}(C=-5 / 3)$ & - & - & -2072 & 5761 & -230647 \\
\hline
\end{tabular}

Source: By the author.

increase in the errors when the order of the Padé is raised.

The Padé was also used to resum the series $\widehat{F}_{L \beta}^{(C=-5 / 3)}$ through the Borel integral. The obtained values are in Fig. 30, where one can see that for $N+M>4$ the results coincide with the true value indicated by the yellow line. The PAs in the new scheme also reproduce the sign-alternating behavior of the original series with increasing $N$, as well as the dominant renormalon $u=-1$ and its residue.

The decay width of the Higgs boson into a pair of bottom quarks is commonly written in the literature in the $\overline{\mathrm{MS}}$ scheme in terms of the scale-dependent quark mass $m(\mu) .{ }^{6,7}$ Then we would like to determine the coefficients of the imaginary part of the scalar correlator in the same way, so we have to reconstruct the results found from the series $\widehat{F}_{L \beta}^{C}$, since it is written as a function of the invariant quark mass. In order to do that, it is necessary to invert Eq. (5.30), that shows the relation between the invariant and the scale-dependent quark mass, and expand it maintaining only the highest terms in $N_{f}$. After that, when $C=0$, i.e., in the $\overline{\mathrm{MS}}$ scheme, one just needs to multiply the Padé series by it.

However, when the PAs were calculated in the scheme with $C=-5 / 3$ we have first to write the results in the $\overline{\mathrm{MS}}$ and later write it as a function of the scale-dependent mass. When expanding Eq. (5.36), one gets the relation below between the coupling in the $\overline{\mathrm{MS}}$ scheme, $a_{s}$, and the one parametrized by a constant $C, \tilde{a}_{s}$

$$
\tilde{a}_{s}=a_{s}-\frac{C \beta_{1}}{2} a_{s}^{2}+\left(\frac{C \beta_{1}}{2}\right)^{2} a_{s}^{3}-\left(\frac{C \beta_{1}}{2}\right)^{3} a_{s}^{4}+\left(\frac{C \beta_{1}}{2}\right)^{4} a_{s}^{5}+\cdots .
$$

When this relation is applied in an expansion like Eq. (5.43), one can reconstruct the result in the $\overline{\mathrm{MS}}$ scheme from the series constructed in another scheme.

In this way, reconstructing the results obtained from the series $\widehat{F}_{L \beta}^{C}$, which is written in 
terms of the invariant mass, we can see in Tab. 1 that the results are better than the ones determined by the Padés to the Borel transform of $F_{L \beta}$. In particular, the coefficients from the PAs using scheme variation were even better, with the Padés $P_{1}^{2}$ and $P_{2}^{2}$ reproducing the sign-alternation, which is not present in any other PA in the table.

However, the predictions are still not satisfactory due to the large errors. So, it is important to explore different approximants, as the partial Padés and the D-log Padés introduced in Sec. 4.3. Since the results obtained from Padés to $\widehat{F}_{L \beta}^{C}$ were the best so far, we will analyze PPAs and D-log Padés to the Borel transform of this series.

Since the imaginary part does not have any double poles, there is no evident gain from using the D-log Padé approximants, whose main characteristic is to transform any pole or branch cut into single poles. Indeed, we tested these approximants in this case and no improvement was observed. Thus we will not show the results coming from the D-log Padés to the Borel transform of $\widehat{F}_{L \beta}^{C}$.

\subsubsection{Partial Padé Approximants to the Imaginary Part in the Large- $\beta_{0}$}

In this section we will apply partial Padé approximants to the reduced imaginary part written as a function of the invariant mass. We will start investigating the contribution of each term of the Borel transform for the coefficients of the perturbative series of $\widehat{F}_{L \beta}^{(C=0)}$ in the large- $\beta_{0}$ limit and in the $\overline{\mathrm{MS}}$ scheme in order to determine which are the best options for the polynomial $T_{k}(u)$ defined in Eq. (4.30). Tab. 2 contains the contribution in percent of the first three UV $(u=-1,-2,-3)$ and IR $(u=3,4,5)$ renormalons and the term which does not have poles. The dominance of the UV pole $u=-1$ starts in the seventh-order coefficient. For the first four coefficients the no-pole term dominates. There are huge cancellations between the contributions for the fifth-order coefficient.

Let us now study the partial Padé approximants, described in Eq. (4.29). We will consider the PPAs of the type $\mathbb{P}_{N, 1}^{M}$. The $T_{1}(u)$ polynomial was built to reproduce the dominant pole of the Borel transform of $\widehat{F}_{L \beta}^{(C=0)}$

$$
T_{1}(u)=(u+1) .
$$

We also examined the PPAs of this kind $\mathbb{P}_{N, 2}^{M}$, where $T_{2}(u)$ reproduces exactly the first two UV poles

$$
T_{2}(u)=(u+1)(u+2) .
$$

The following near-diagonal Padé sequences were analyzed: $\mathbb{P}_{N, 1}^{N}, \mathbb{P}_{N, 1}^{N+1}, \mathbb{P}_{N+1,2}^{N}$ and $\mathbb{P}_{N, 2}^{N+2}$. The Padé $\mathbb{P}_{2,1}^{2}$, after being reconstructed in terms of the scale-dependent mass, predicts the coefficient $c_{6}$ of $F_{L \beta}$ with an accuracy of $67 \%$, which is half of the error obtained from $P_{2}^{2}$ in the $\overline{\mathrm{MS}}$ scheme, and it also recreates the residue for the dominant pole $u=-1$ with an associated error of $30 \%$. This PPA also has the sign-alternating behavior that was not manifested before in the standard Padé. 
Table 2 - Contribution in percent of the lowest UV and IR poles and the no-pole term to the first 12 coefficients of the $\widehat{F}_{L \beta}^{(C=0)}$ series in the $\overline{\mathrm{MS}}$ scheme.

\begin{tabular}{ccccccc}
\hline & $\widehat{c}_{1}$ & $\widehat{c}_{2}$ & $\widehat{c}_{3}$ & $\widehat{c}_{4}$ & $\widehat{c}_{5}$ & $\widehat{c}_{6}$ \\
\hline $\mathrm{UV}_{-1}$ & $18.2 \%$ & $-6.2 \%$ & $-18.5 \%$ & $-83.9 \%$ & $381.3 \%$ & $17.3 \%$ \\
$\mathrm{UV}_{-2}$ & $-4.5 \%$ & $-3.1 \%$ & $9.2 \%$ & $44.2 \%$ & $-28.5 \%$ & $24.1 \%$ \\
$\mathrm{UV}_{-3}$ & $2.0 \%$ & $2.1 \%$ & $-3.6 \%$ & $-28.4 \%$ & $-13.0 \%$ & $-13.7 \%$ \\
$\mathrm{IR}_{3}$ & $-2.0 \%$ & $-4.8 \%$ & $-4.1 \%$ & $29.3 \%$ & $268.5 \%$ & $-37.5 \%$ \\
$\mathrm{IR}_{4}$ & $1.1 \%$ & $2.5 \%$ & $1.5 \%$ & $-19.7 \%$ & $-138.8 \%$ & $13.5 \%$ \\
$\mathrm{IR}_{5}$ & $-0.7 \%$ & $-1.5 \%$ & $-0.7 \%$ & $13.5 \%$ & $82.3 \%$ & $-5.9 \%$ \\
No-pole & $86.4 \%$ & $111.3 \%$ & $114.9 \%$ & $139.7 \%$ & $-429.8 \%$ & $95.3 \%$ \\
\hline Total & $100.5 \%$ & $100.3 \%$ & $98.7 \%$ & $94.7 \%$ & $122.0 \%$ & $93.1 \%$ \\
\hline & $\widehat{c}_{7}$ & $\widehat{c}_{8}$ & $\widehat{c}_{9}$ & $\widehat{c}_{10}$ & $\widehat{c}_{11}$ & $\widehat{c}_{12}$ \\
\hline $\mathrm{UV}_{-1}$ & $156.3 \%$ & $100.7 \%$ & $98.2 \%$ & $99.6 \%$ & $100.4 \%$ & $99.9 \%$ \\
$\mathrm{UV}_{-2}$ & $-16.5 \%$ & $-3.4 \%$ & $2.6 \%$ & $-0.3 \%$ & $-0.2 \%$ & $0.1 \%$ \\
$\mathrm{UV}_{-3}$ & $14.8 \%$ & $1.2 \%$ & $-1.8 \%$ & $0.3 \%$ & $0.1 \%$ & $0.0 \%$ \\
$\mathrm{IR}_{3}$ & $6.5 \%$ & $11.8 \%$ & $-7.6 \%$ & $1.9 \%$ & $-0.1 \%$ & $-0.1 \%$ \\
$\mathrm{IR}_{4}$ & $9.7 \%$ & $-8.8 \%$ & $3.5 \%$ & $-0.2 \%$ & $-0.3 \%$ & $0.1 \%$ \\
$\mathrm{IR}_{5}$ & $-9.8 \%$ & $5.6 \%$ & $-1.6 \%$ & $-0.2 \%$ & $0.3 \%$ & $-0.1 \%$ \\
No-pole & $-59.0 \%$ & $-4.6 \%$ & $5.5 \%$ & $-1.1 \%$ & $-0.1 \%$ & $0.1 \%$ \\
\hline Total & $102.0 \%$ & $102.5 \%$ & $98.8 \%$ & $100.0 \%$ & $100.1 \%$ & $100.0 \%$ \\
\hline
\end{tabular}

Source: By the author.

After reconstructing all the PPAs as a function of the scale-dependent quark mass $m(\mu)$, we plotted Fig. 31, that shows the relative errors for the first predicted coefficient $c_{n}$ for each PPA. As we can see, the errors did not have a significant reduction compared to the ones found using the standard Padés in the $\overline{\mathrm{MS}}$ scheme (the plot is in the same scale as Fig. 27) for lower orders, i.e., for $N+M \leq 3$, because at these orders there is no evident dominance of any renormalon.

However, the Borel integral computed from the PPAs is very close to the real one, as indicated in Fig. 32, which illustrates the real Borel sum in yellow and the estimates from the partial Padés. Most of the PPAs have the sign-alternating behavior of $\widehat{F}_{L \beta}^{(C=0)}$, which was not present in the standard Padés and is a consequence of the imposition of the UV poles. In addition, they reproduce the residue of the pole $u=-1$ with increasing $N$, although they did not mimic any other renormalons of the Borel transform or even the residue of $u=-2$ (for $\mathbb{P}_{N+1,2}^{N}$ and $\mathbb{P}_{N, 2}^{N+2}$ ).

Changing the renormalization scheme to $C=-5 / 3$ accelerates the convergence. For the PPA $\mathbb{P}_{2,1}^{2}$ in this scheme, the error of the estimate of the coefficient $c_{6}$ (of the function $F_{L \beta}$ in the $\overline{\mathrm{MS}}$ scheme) is just $4.5 \%$, one order lower than the PPAs in the $\overline{\mathrm{MS}}$. The sign-alternation is present even when the function is reconstructed in terms of the scaledependent mass. In addition it also predicts the residue of the leading pole $u=-1$ with an error of just $3 \%$. This corroborates the idea that the PPAs in the $\overline{\mathrm{MS}}$ scheme do 


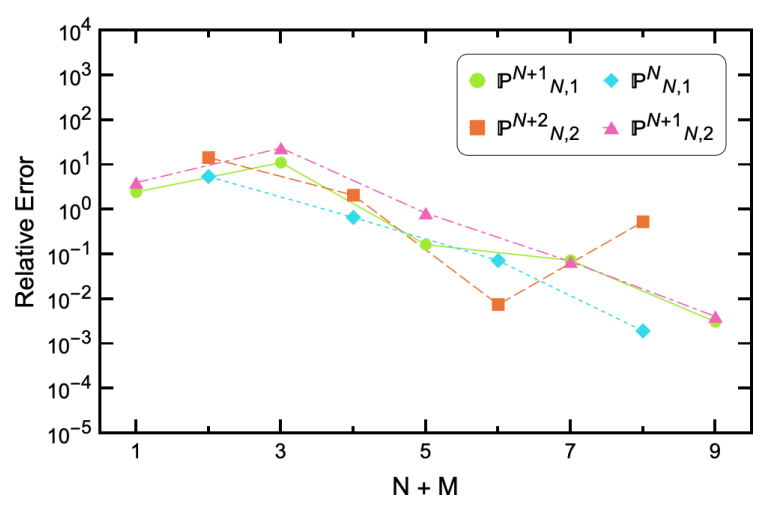

Figure 31 - Relative error for the first predicted coefficient $c_{n}$ of $F_{L \beta}$ for each PPA after being rebuilt as a function of $m(\mu)$.

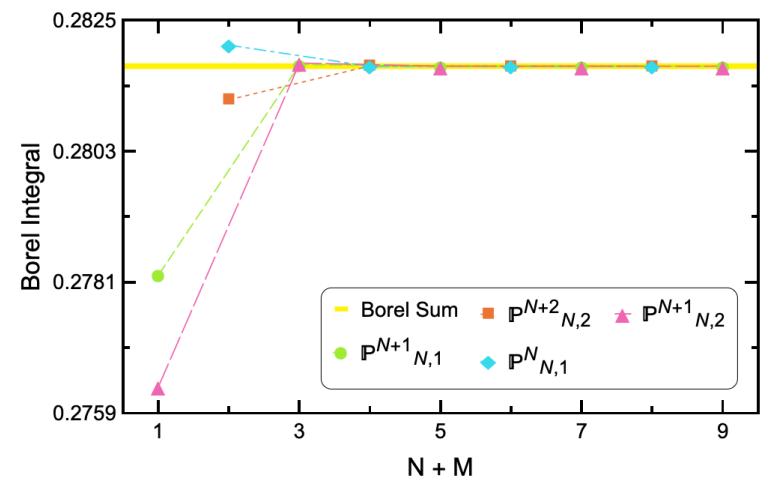

Figure 32 - Borel integral of $\widehat{F}_{L \beta}^{(C=0)}$ for PPAs to the imaginary part in the large$\beta_{0}$ limit using $\alpha_{s}\left(m_{H}\right)=0.1125$.

Source: By the author.

not improve much because no pole dominates at lower orders. For $C<0$ then $u=-1$ dominates and the use of the partial Padés becomes advantageous.

The relative errors of the first predicted coefficient $c_{n}$ for all the PPAs studied in this scheme, after being brought to the $\overline{\mathrm{MS}}$ scheme as a function of the scale-dependent quark mass, are indicated in Fig. 33. It is possible to notice that the errors are one to two orders lower than the ones obtained from the PPAs in the usual scheme. The residues of the poles added "by hand" through $T_{k}(u=-1$ and $u=-2)$ are reproduced even in PPAs of lower order ( small $N)$. However, in this scheme the partial Padés did not have a great improvement at lower orders compared to the standard Padés in the same scheme, where $C=-5 / 3$, and, since the partial Padé approximants are model-dependent, the Padé approximants with scheme variation are still the best option.

The reason for the little improvement of the Padés at lower orders when compared to the standard Padés is because, as we can notice in Tab. 2, the function is more dominated by the no-pole term until the fourth-order coefficient, so the addition of the UV poles for Padés with few coefficients as initial parameters would not interfere much in the results.

Since the errors are still large when considering the partial and standard Padés that use the same number of coefficients known in QCD, we will analyze Padé approximants to other functions in the large- $\beta_{0}$ limit, as the scalar correlator and its second derivative. The results achieved from the Borel transform of the scalar correlator, given in Eq. (5.21), were not so good as the ones from the imaginary part. This occurred because of the structure of the Borel transform: the dominant renormalon is at $u=1$, so the series is fixed-sign, unlike the series of the imaginary part. This IR renormalon is also responsible for the fast increase of the coefficients of the scalar correlator compared to the imaginary part or the second derivative. ${ }^{59}$ It also has an infinite number of double poles (only the renormalons $u=1$ and $u=2$ are simple), which is more difficult for the PA to approximate; the Padé spends 


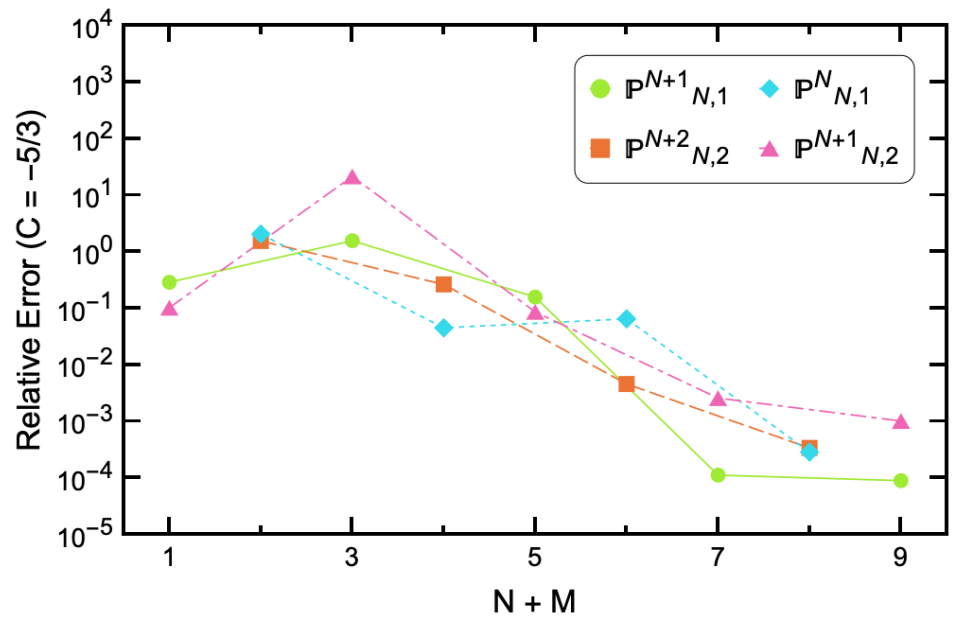

Figure 33 - Relative error for the first predicted coefficient $c_{n}$ of $F_{L \beta}$ in the $\overline{\mathrm{MS}}$ for each PPA constructed in the scheme with $C=-5 / 3$ after being rebuilt as a function of $m(\mu)$.

Source: By the author.

more coefficients "trying" to mimic the double poles, so the results get worse. Besides that, this function in QCD has a great disadvantage when compared to the imaginary part or the second derivative: the perturbative series for $\mu^{2}=-s$, i.e., when the logarithms are summed, is known only up to third order. ${ }^{60}$ For these reasons we are not going to show explicitly the outcomes of these Padé approximants.

\subsection{Padé Approximants to the Second Derivative in the Large- $\boldsymbol{\beta}_{0}$}

In this section, we will apply Padés to the Borel transform of the second derivative, indicated in Eq. (5.42). We will work with $\Pi_{L \beta}^{\prime \prime}$ written in terms of the invariant mass $\widehat{m}$ since it presented the best results for the imaginary part. The perturbative coefficients of the reduced second derivative $\widehat{D}_{L \beta}^{C}$ in the $\overline{\mathrm{MS}}$ are in Eq. (5.35) and the double poles $u=-1$ and $u=-2$ and the simple one $u=2$ are the dominant renormalons of this function. The sign-alternating behavior sets in at the sixth-order coefficient in the $\overline{\mathrm{MS}}$ scheme showing the dominance of the UV renormalon $u=-1$ at intermediate orders.

We will again study the Padé $P_{1}^{1}$ at the $\overline{\mathrm{MS}}$ scheme, which is given by

$$
P_{1}^{1}(u)=\frac{1.4949-1.9035 u}{0.5372-u}
$$

and the first predicted coefficient, $\widehat{r}_{4}$, has a relative error of 593, which is extremely large. The pole of this Padé is not associated with any renormalon of the Borel transform and it does not recreate the sign-alternating behavior of $\widehat{D}_{L \beta}^{(C=0)}$. The next Padé of this sequence, $P_{2}^{2}$, indicated below

$$
P_{2}^{2}(u)=\frac{3.4728+3.5262 u+1.8913 u^{2}}{(0.8819+u)(1.4152-u)}
$$




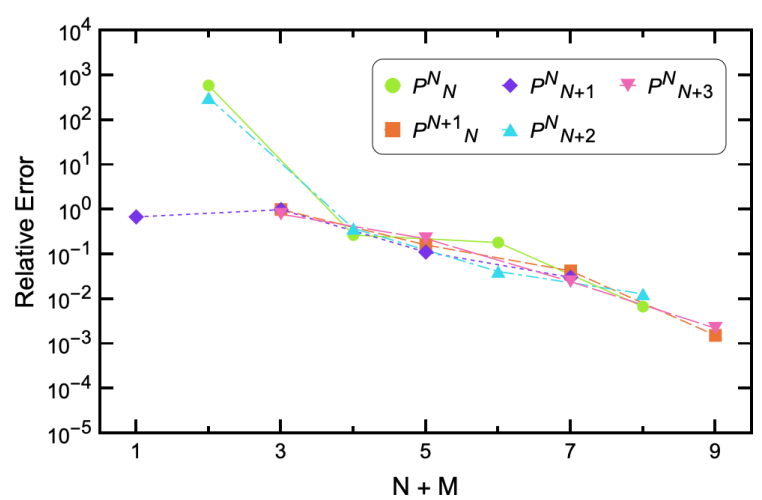

Figure 34 - Relative error for the first predicted coefficient $\widehat{r}_{n}$ for each Padé to the second derivative.

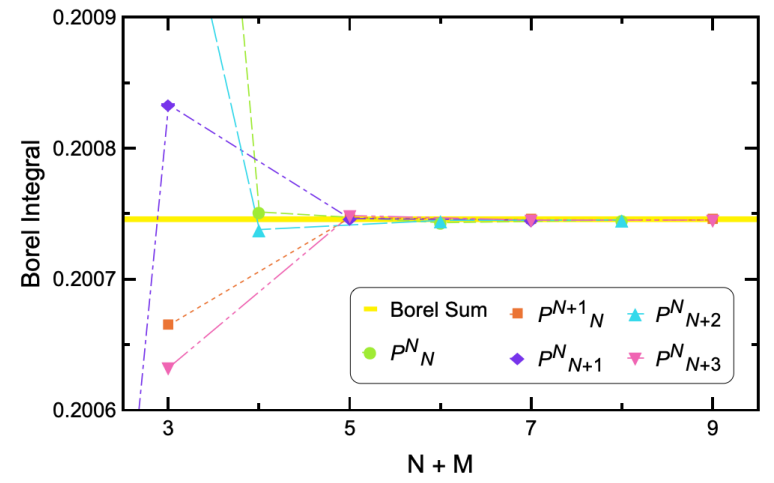

Figure 35 - Borel integral of $\widehat{D}_{L \beta}^{(C=0)}$ for Padés to the second derivative in large- $\beta_{0}$ with $\alpha_{s}\left(m_{H}\right)=0.1125$.

Source: By the author.

improved a lot the results. The estimate for the first predicted coefficient, $\widehat{r}_{6}$, is -6148 , which has to be compared with the real value -8410.57 : this represents an error of just $27 \%$. One can also notice that the Padé has a pole close to the dominant renormalon $u=-1$ and another one close to the first IR pole $u=2$. The sign-alternating behavior of the series $\widehat{D}_{L \beta}^{(C=0)}$ is also present in this Padé.

As before, we analyzed also the following Padé sequences: $P_{N}^{N+1}, P_{N+1}^{N}, P_{N+2}^{N}$ and $P_{N+3}^{N}$; and the relative error, defined in Eq. (4.33), between the first predicted coefficient and the exact value of $\widehat{r}_{n}$ for all the PAs is shown in Fig. 34. The Padés converge when $N$ is increased as expected, but the errors are large considering the Padé approximants with less than five coefficients as initial parameters.

Despite the big errors, the PAs mimic the dominant pole $u=-1$, with the right multiplicity just for higher values of $N$, and the first IR renormalon $u=2$. However, the estimated residues for these poles are not close to the real ones. Most of the Padés reproduce the sign-alternating behavior of $\widehat{D}_{L \beta}^{(C=0)}$ and its Borel integral, which can be noted in Fig. 35, that illustrates the true value in yellow and the ones computed using the Padé approximants. For $N+M>4$ the estimates coincide with the real integral.

The renormalization scheme was also changed to $C=-5 / 3$ and we expect better predictions in this new scheme, since the exponential of $\widehat{D}_{L \beta}^{C}$ is canceled and the UV renormalon is more dominant, and indeed we can observe an improvement. The same Padé sequences were analyzed and all of them were reconstructed in the $\overline{\mathrm{MS}}$ scheme using Eq. (5.44). The relative error due to the first predicted coefficient $\widehat{r}_{n}$ in $\overline{\mathrm{MS}}$ is around one to two orders of magnitude smaller, although for lower orders the error is still large. All the Padés have the sign-alternating coefficients, but they did not estimate correctly the residues of the poles $u=-1$ and $u=2$ that are reproduced by the PAs when $N$ is raised.

Since the errors are still very high when considering just the Padé approximants that use the first four coefficients as initial parameters, we will investigate other methods, as 
the D-log Padé approximants.

\subsubsection{D-log Padés to the Second Derivative in the Large- $\beta_{0}$}

In realistic situations we rarely know the position of the poles and its multiplicities, and some of the functions have cuts. Thus, a method that can be more effective in these conditions are the D-log Padés defined in Sec. 4.3. We will work at this point with the D-log Padés to the Borel transform of the series $\widehat{D}_{L \beta}^{C}$, indicated in Eq. (5.42).

As already discussed, the D-log Padé is not a PA to the function $f(z)$, but to $F(z)=$ $\frac{\mathrm{d}}{\mathrm{d} z} \ln f(z)$. From the coefficients of the D-log Padé, considering that $f(z)$ has one singularity at $z=\mu$, one can then obtain the location of the singularity as well as its multiplicity.

Let us begin examining $\operatorname{Dlog}_{1}^{1}$ in the $\overline{\mathrm{MS}}$ scheme, whose expression is

$$
\operatorname{Dlog}_{1}^{1}(u)=\frac{64}{23} \frac{1}{(1.0734+u)^{2.1238}} .
$$

After expanding it, the first predicted coefficient of $\operatorname{Dlog}_{1}^{1}$ is $\widehat{r}_{5}^{P}=1127$ with an error of just $25 \%$, which is very small compared to the ones found before. This D-log also has the sign-alternating behavior and it predicts rather precisely the leading renormalon of the Borel transform, the double pole at $u=-1$. Analyzing the next Padé in this sequence, $\operatorname{Dlog}_{2}^{2}$, the first predicted coefficient, $\widehat{r}_{7}^{P}=189406$, is also in good agreement with the real one: it is off by $16 \%$. This D-log Padé also has a pole close to the dominant one, at $u=-0.83$, but its multiplicity is not correct, $\gamma=1.10$, half of the real value.

Fig. 36 illustrates the relative error of the first coefficient predicted by each D-log Padé sequence analyzed and we can see that the relative errors decrease when the order of the D-log is raised and they are lower than the standard Padé ones. The increase in the error with higher $N$ is due to poles distant from the origin or defects (poles almost canceled by a close-by zero) which effectively reduce the order of the D-log Padé.

Analyzing just the D-log Padés that uses the first four coefficients as input, i.e., that predicts the coefficient of $\alpha_{s}^{5}$, one can notice that $\operatorname{Dlog}_{2}^{0}$ has a relative error approximately two orders of magnitude higher than $\operatorname{Dlog}_{0}^{2}$ and $\operatorname{Dlog}_{1}^{1}$. The reason for that is in the standard Padé $P_{2}^{0}$ used to build the D-log. The expression for $P_{2}^{0}$ is

$$
P_{2}^{0}(u)=\frac{0.0461}{(0.1230-0.2517 i-u)(0.1230+0.2517 i-u)},
$$

and it is possible to observe that it has a pair of complex poles close to the origin. As we discussed in Sec. 4.3, the complex poles can appear when the function to be approximated is meromorphic, however the Padé approximation to the function breaks down close to these poles. Thus, the estimates of $\operatorname{Dlog}_{2}^{0}$ can be disconsidered since $P_{2}^{0}$ is not a reliable approximation for the function $F(z)$ defined in Eq. (4.35).

The Borel integrals estimated by the D-log Padés, which is shown in Fig. 37, are very close to the real value in yellow and the estimates get better with greater $N$. It is possible 


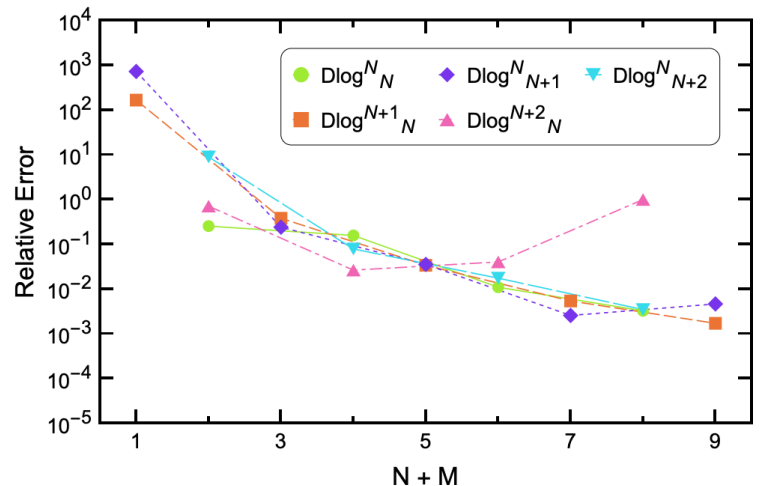

Figure 36 - Relative error for the first predicted coefficient $\widehat{r}_{n}$ of $\widehat{D}_{L \beta}^{(C=0)}$ for each D-log Padé to the second derivative.

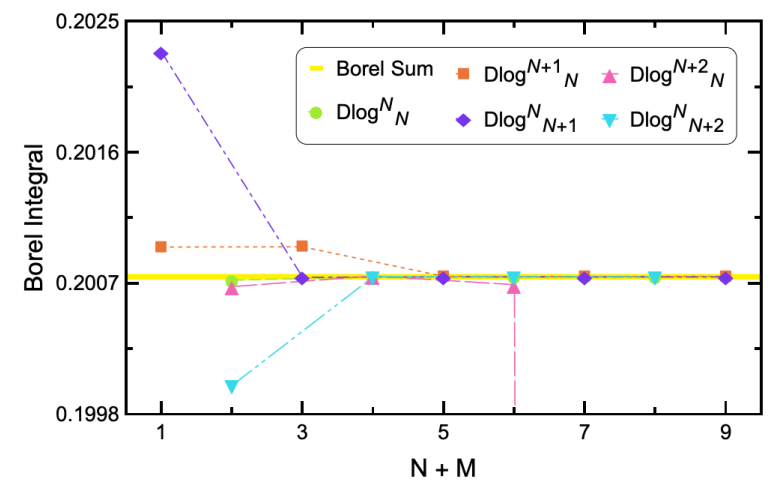

Figure 37 - Borel integral of $\widehat{D}_{L \beta}^{(C=0)}$ for Dlog Padés to the second derivative in the large- $\beta_{0}$ limit with $\alpha_{s}\left(m_{H}\right)=0.1125$.

Source: By the author.

to notice that when the order of the D-log Padé is increased, the sign-alternating behavior and the dominant pole $u=-1$ are replicated, although not with the right multiplicity.

We also changed the renormalization scheme to $C=-5 / 3$, where the UV pole dominates more the series behavior. The results are expected to be better since the exponential vanishes and the sign-alternating behavior of the series starts earlier in this scheme. In the new scheme, $\operatorname{Dlog}_{1}^{1}$ replicates the sign-alternation and the error for the predicted coefficient $\widehat{r}_{5}$ is now $9.3 \%$, more than half as small as the $\overline{\mathrm{MS}}$ estimate. However the pole estimate is not as good as the other one: $\operatorname{Dlog}_{1}^{1}$ has a pole at $u=2.04$ with a multiplicity of $\gamma=17.80$. The next D-log Padé of this sequence $\operatorname{Dlog}_{2}^{2}$ has a defect: one of its poles is at $u=0.463$ and one zero is at $u=0.449$, thus its results are not reliable.

The D-log Padés were reconstructed in the $\overline{\mathrm{MS}}$ scheme and the relative errors of the first predicted coefficient is exhibited in Fig. 38 for all the sequences studied. They are a little lower when compared to the errors in the $\overline{\mathrm{MS}}$ scheme given in Fig. 36 and they reduce with increasing $N$ for most of the sequences. In the cases where the error grows is due to the appearance of the defects. With increasing $N$, one can observe that the D-log Padés replicate the sign-alternating coefficients and the dominant renormalon of the second derivative as well as its multiplicity.

Considering the D-log Padés that can be applied in QCD to predict the first unknown coefficient, the estimates from the D-log in the scheme with $C=-5 / 3$ are more accurate for $\operatorname{Dlog}_{1}^{1}$ and $\operatorname{Dlog}_{0}^{2}$ when compared to the ones in the $\overline{\mathrm{MS}}$ scheme. The predictions for $\operatorname{Dlog}_{2}^{0}$ are worse and the cause is the same as the $\overline{\mathrm{MS}}$ one: the Padé $P_{2}^{0}$ has a pair of complex poles in close proximity to the origin, at $u=0.0206 \pm 0.0362 i$, that ruins the convergence of the D-log Padé.

One can notice that the results for the D-log Padés which estimate the third-order coefficient, $\operatorname{Dlog}_{1}^{0}$ and $\operatorname{Dlog}_{0}^{1}$, are not good even when the scheme is changed to a less 


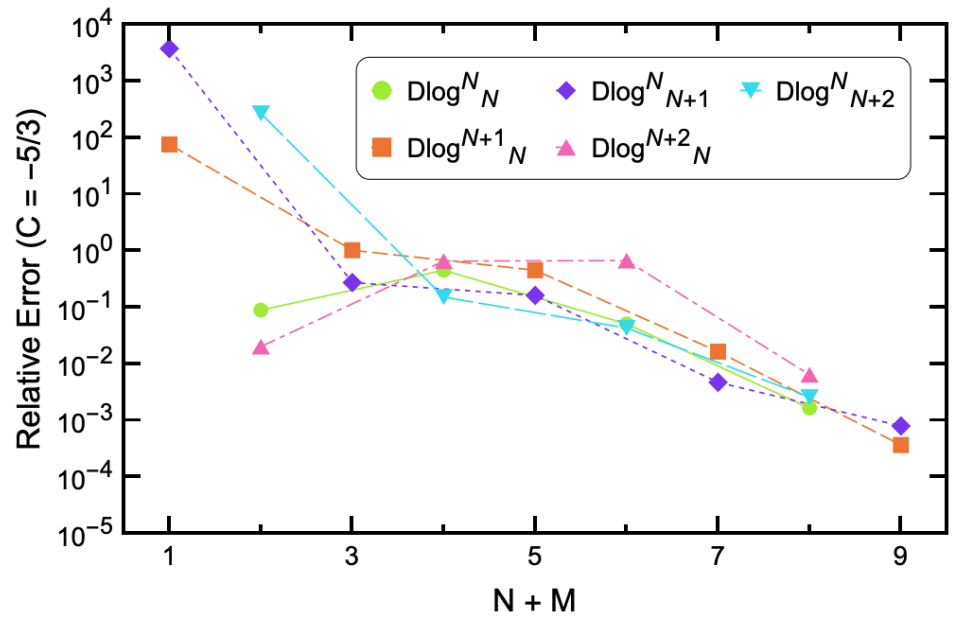

Figure 38 - Relative error for the first predicted coefficient $\widehat{r}_{n}$ of $\widehat{D}_{L \beta}^{(C=0)}$ in the $\overline{\mathrm{MS}}$ scheme for each D-log Padé to the second derivative constructed in the scheme where $C=-5 / 3$.

Source: By the author.

perturbative one. A reason for this is because they use very little information as input, which is not enough for them to replicate the main characteristics of the reduced second derivative. In addition, the Padés $P_{1}^{0}$ and $P_{0}^{1}$, which are used to obtain those D-log Padés, do not approximate reasonably well any function.

As we can see, the errors obtained from the D-log Padés are much smaller compared to the standard Padés. This can be explained by the fact that the Borel transform in Eq. (5.42) has plenty of double poles, which is more difficult for the PA to approximate. However, the D-log Padé, as we shown in Sec. 4.3, transforms the double poles into single poles, thus it is expected that the results should be better.

\subsection{Calculating the Coefficients $d_{n, 1}$}

After we calculate the Padé approximants to the imaginary part and to the second derivative, we need to compare both results in order to determine the best strategy to apply in QCD. As we discussed in Ch. 3, the only independent coefficients of the scalar correlator are the $d_{n, 1}$ of Eq. (3.52), and it is possible to determine them from the coefficients $c_{n}$ of the imaginary part and $r_{n}$ of the second derivative. Hence, after computing the $d_{n, 1}$ we would be able to analyze all the results in the large- $\beta_{0}$ limit and compare them.

First we will compute the relation between the coefficients $d_{n, 1}$ and $c_{n}$ of the imaginary part. In Ch. 3 we saw that, when $\operatorname{Im} \Pi$ is written in terms of the scale-dependent mass, as in Eq. (3.54), the coefficients $c_{n}$ are defined as

$$
c_{n}=\sum_{k=0}^{[n / 2]} d_{n, 2 k+1}(i \pi)^{2 k},
$$

where $[n]$ is the integer value of $n$. This relation is also valid in the large- $\beta_{0}$ limit, the 
value of the coefficients that differs from QCD to large- $\beta_{0}$. Through the RGE of the scalar correlator, given in Eq. (3.51), we can determine in QCD the following relations between the coefficients $d_{n, k}$ and $d_{n, 1}$

$$
\begin{aligned}
& d_{1,2}=-\frac{1}{2} d_{0,1} \gamma_{m}^{(1)} \\
& d_{n, 2}=-\frac{1}{2} d_{0,1} \gamma_{m}^{(n)}-\frac{1}{4} \sum_{m=1}^{n-1}\left(m \beta_{n-m}+2 \gamma_{m}^{(n-m)}\right) d_{m, 1}, \\
& d_{n, k+1}=-\frac{1}{2(k+1)} \sum_{m=1}^{n-1}\left(m \beta_{n-m}+2 \gamma_{m}^{(n-m)}\right) d_{m, k} .
\end{aligned}
$$

In the large- $\beta_{0}$ limit, the $\beta$ function is truncated at first order, so the relations above can be rewritten as

$$
\begin{aligned}
& d_{1,2}=-\frac{1}{2} d_{0,1} \gamma_{m}^{(1)} \\
& d_{n, 2}=-\frac{1}{2} d_{0,1} \gamma_{m}^{(n)}-\frac{(n-1)}{4} \beta_{1} d_{n-1,1}, \\
& d_{n, k+1}=-\frac{(n-1)}{2(k+1)} \beta_{1} d_{n-1, k} .
\end{aligned}
$$

Then, using Eqs. (5.45) and (5.47) we can evaluate the coefficients $d_{n, 1}$ predicted by the Padés applied to the Borel transform of the imaginary part in the large- $\beta_{0}$ limit.

Let us calculate now the coefficients $d_{n, 1}$ through the coefficients $r_{n}$. As we saw, the second derivative of the scalar correlator, for $\mu^{2}=-s$ shown in Eq. (3.58), has the following coefficients: $r_{n}=d_{n, 1}+2 d_{n, 2}$, which is also valid in the large- $\beta_{0}$ limit, the only difference are the values of the coefficients. The relation between $d_{n, 2}$ and $d_{n, 1}$ is indicated in Eq. (5.46), and, using it, we obtain in QCD the expression below between the coefficients of the second derivative $r_{n}$ and $d_{n, 1}$

$$
d_{n, 1}=r_{n}-2 d_{n, 2}=r_{n}+d_{0,1} \gamma_{m}^{(n)}+\frac{1}{2} \sum_{m=1}^{n-1}\left(m \beta_{n-m}+2 \gamma_{m}^{(n-m)}\right) d_{m, 1} .
$$

Employing instead Eq. (5.47) the relation in the large- $\beta_{0}$ limit can be determined as follows

$$
d_{n, 1}=r_{n}+d_{0,1} \gamma_{m}^{(n)}+\frac{(n-1)}{2} \beta_{1} d_{n-1,1} .
$$

This is the expression to determine the coefficients $d_{n, 1}$ from the estimates of the Padé approximants to the Borel transform of the second derivative.

At this point we can calculate the coefficients $d_{n, 1}$ predicted by the Padés to the imaginary part and to the second derivative, in order to compare both results and determine the best method to apply in QCD. Since the Padé approximants were applied to the Borel transform of the function written in terms of the invariant quark mass, first we will have to reconstruct the coefficients of the function in terms of the scale-dependent 
Table 3 - Coefficients $d_{n, 1}$ in the $\overline{\mathrm{MS}}$ predicted by Padés and D-log Padés and the exact value in large- $\beta_{0}$ in blue. The Padés in white were applied to the Borel transform of $\widehat{F}_{L \beta}^{C}$ and the ones in pink to the Borel transform of $\widehat{D}_{L \beta}^{C}$. The PAs with $(C)$ were constructed in the scheme where $C=-5 / 3$, otherwise they were applied in the $\overline{\mathrm{MS}}$ scheme.

\begin{tabular}{cccccc}
\hline & $d_{4,1}$ & $d_{5,1}$ & $d_{6,1}$ & $d_{7,1}$ & $d_{8,1}$ \\
\hline Large- $\beta_{0}($ exact $)$ & 690 & 6798 & 56756 & 816323 & $8.86 \times 10^{6}$ \\
$P_{1}^{1}$ & 765 & 7238 & 69978 & 908534 & $1.18 \times 10^{7}$ \\
$P_{1}^{1}(C)$ & 733 & 6939 & 63790 & 836905 & $1.03 \times 10^{7}$ \\
$P_{2}^{0}$ & 720 & 6689 & 59410 & 767080 & $9.22 \times 10^{6}$ \\
$P_{2}^{0}(C)$ & 735 & 6952 & 64156 & 839532 & $1.05 \times 10^{7}$ \\
$P_{2}^{1}$ & - & 6187 & 50006 & 623405 & $6.81 \times 10^{6}$ \\
$P_{3}^{0}(C)$ & - & 6766 & 57730 & 813005 & $9.22 \times 10^{6}$ \\
$P_{1}^{1}$ & 1148 & 15350 & 263987 & 582750 & $7.82 \times 10^{6}$ \\
$P_{1}^{1}(C)$ & 468 & 8354 & -15825 & $2.23 \times 10^{6}$ & $-4.05 \times 10^{7}$ \\
$P_{2}^{0}$ & 934 & 9669 & 120300 & $1.78 \times 10^{6}$ & $3.02 \times 10^{7}$ \\
$P_{2}^{0}(C)$ & 527 & 7898 & 8277 & $1.68 \times 10^{6}$ & $-1.98 \times 10^{7}$ \\
$P_{1}^{2}(C)$ & - & 6865 & 52475 & 844916 & $7.06 \times 10^{6}$ \\
$P_{2}^{1}$ & - & 8301 & 54952 & $1.40 \times 10^{6}$ & $3.92 \times 10^{6}$ \\
$P_{3}^{0}$ & - & 5607 & 36031 & 320352 & 994676 \\
$P_{3}^{0}(C)$ & - & 7513 & 65794 & $1.07 \times 10^{6}$ & $1.33 \times 10^{7}$ \\
$\operatorname{Dlog}_{0}^{2}$ & - & 5722 & 49141 & 573274 & $7.31 \times 10^{6}$ \\
$\operatorname{Dlog}_{0}^{2}(C)$ & - & 6768 & 58331 & 787367 & $9.35 \times 10^{6}$ \\
$\operatorname{Dlog}_{1}^{1}$ & - & 6412 & 54721 & 731481 & $8.48 \times 10^{6}$ \\
$\operatorname{Dlog}_{1}^{1}(C)$ & - & 6933 & 59579 & 842463 & $9.80 \times 10^{6}$ \\
\hline
\end{tabular}

Source: By the author.

mass using Eq. (5.30) and then calculate the coefficients $d_{n, 1}$.

The estimated coefficients $d_{n, 1}$ are summarized in Tab. 3 , where the values in blue are the exact ones in the large- $\beta_{0}$ limit, the ones in white were applied to the reduced imaginary part and the ones in pink to the reduced second derivative. All the Padés and D-log Padés that predict the fourth- or fifth-order coefficients are indicated in Tab. 3, unless they have a defect, a pole far away from the origin or a pair of complex poles near the origin, which make their estimates unreliable. The results for $D \log _{1}^{0}$ and $D \log _{0}^{1}$ are not presented as well because we concluded they are not good approximants in this case. Since the partial Padés did not improve much the predictions compared to the PAs and we want a technique as model-independent as possible, we will not show their estimates.

Analyzing the predicted coefficients $d_{n, 1}$, one can notice that the errors are considerably smaller than the ones in the previous sections. This occurred because, as we saw, the coefficients $c_{n}$ and $r_{n}$, and consequently $\widehat{c}_{n}$ and $\widehat{r}_{n}$, depend on $d_{n, k}$, which are all related to $d_{n, 1}$. Let us take as an example the relation between $d_{n, 1}$ and $r_{n}$ given in Eq. (5.48). As we can notice, the second and third terms $\left(d_{0,1} \gamma_{m}^{(n)}+\frac{(n-1)}{2} \beta_{1} d_{n-1,1}\right)$ are exactly known, hence the uncertainty of $d_{n, 1}$ comes from $r_{n}$, which represents just a fraction of $d_{n, 1}$. Thus, 
it is expected that the errors before (where we were investigating the deviations due to the predictions of $\widehat{c}_{n}$ and $\widehat{r}_{n}$ ) are higher.

It is possible to infer that the standard Padé estimates are superior when applied to the Borel transform of the reduced imaginary part, $\widehat{F}_{L \beta}^{C}$. The reduced second derivative, $\widehat{D}_{L \beta}^{C}$, has many double poles, including the leading one $u=-1$, thus the PAs spend more coefficients "trying" to mimic them, making the estimates worse. The results from the Borel transform of $\widehat{F}_{L \beta}^{C}$ are even better when considering the scheme $C=-5 / 3$, since now the series is more dominated by the first UV pole and the exponential term is eliminated.

In addition the D-log Padés to the second derivative produce the most effective predictions, especially for $C=-5 / 3$. The double poles of the Borel transform of $\widehat{D}_{L \beta}^{C}$, which were a problem for the standard Padés, are transformed into single poles through the function $F(z)$ defined in Eq. (4.35). Hence, the series is simpler to be approximated.

\subsection{Partial Conclusions}

In this section we summarize the main conclusions that can be drawn from the results of the Padé method in the large- $\beta_{0}$ limit.

The Padé approximants to the Borel transform of the imaginary part written in terms of the scale-dependent quark mass $m(\mu)$ exhibit convergence. There are a few cases though that raising the order of the Padé does not improve the results and we were able to explain in some examples that the presence of the so-called defects causes this phenomenon. Other behavior observed that spoils the Padé predictions is poles far from the origin due to the reduction of the order of the Padé. With seven or eight coefficients, the PA can reproduce very well the leading poles of the Borel transform, the Borel integral, the sign-alternating behavior and the higher-order coefficients. However, the results using just four coefficients, the same amount we know in QCD, are not impressive, so other techniques were tested.

The first one is to change the renormalization scheme of the strong coupling. For less perturbative schemes, where $C$ is negative, the PA can make better predictions. The sign-alternation and the dominant pole are replicated earlier, with fewer coefficients used as initial parameters. This occurred because the Borel transform in the new scheme is more dominated by the UV pole, which can be observed in the sign-alternating behavior of the series that sets in earlier, and the exponential term is eliminated. The partial Padé approximants were calculated as well, but they did not improve much the results at lower orders when compared to the ones obtained using scheme variation because the reduced imaginary part is highly dominated by the no-pole term until the fourth-order coefficient.

The Padé approximants applied to the second derivative of the scalar correlator were also analyzed. The results from the standard PAs are worse compared to the imaginary part, even when the scheme is changed. This worsening is due to the tower of double poles present in the Borel transform of $\widehat{D}_{L \beta}^{C}$, which forces the Padé to spend coefficients 
"trying" to mimic these renormalons. However, the D-log Padés to the second derivative got impressive results, being the best strategy so far considering the small number of known parameters. This can be explained by the fact that the double poles are turned into single poles, which simplifies the function to be approximated. We also showed that $\operatorname{Dlog}_{1}^{0}$ and $\operatorname{Dlog}_{0}^{1}$ are not good approximants for the second derivative, because the standard Padés used to build them are basically a monopole and a straight line respectively. It was observed as well that complex poles generate unreliable estimates when they are located close to the origin.

Summarizing, the Padés and D-log Padés that use just the first three coefficients as initial parameters, i.e., predicts the coefficient of $a_{s}^{4}$, are not accurate. There is simply not enough information to reliably estimate the coefficient and the series characteristics at this order. However, when four coefficients are used as input the predictions improve and the method with the best results are the D-log Padés to the second derivative. The standard Padés to the imaginary part allied with renormalization scheme variation were also good.

Studying the use of Padé approximants in the large- $\beta_{0}$ limit provided us good techniques to determine the higher-order coefficients more precisely. In the next chapter we will employ our findings to the perturbative series in QCD. 


\section{RESULTS IN QCD}

In the last chapter we studied the use of Padé approximants in the large- $\beta_{0}$ limit in order to develop a strategy that could be applied to QCD. The D-log Padé approximants to the Borel transform of the second derivative proved to be efficient with the number of coefficients we have in QCD. We also concluded that the Padé approximants to the Borel transform of the imaginary part associated with scheme variation improved a lot the estimates of the first unknown coefficient as well as the convergence to the original series.

In QCD, the renormalons of the perturbative series are at the same position of the renormalons in the large- $\beta_{0}$ limit, however they are now branch cuts, not isolated poles. ${ }^{9}$ Even though there are no theorems that state the convergence of the Padé approximants to series with branch cuts, in many practical applications, as the one showed in Sec. 4.3, there are indications that this convergence happens. ${ }^{18}$

In this chapter we will obtain the higher-order QCD corrections to the decay rate of the Higgs into bottom quarks using model-independent techniques. We will analyze the Padé and the D-log Padé approximants to the Borel transform of the reduced imaginary part and second derivative in QCD (we remind that the reduced function is the one that contains only the non-trivial part of the perturbative series, i.e. the $a_{s}$ corrections). We will also explore scheme variations, since it presented great results in the large- $\beta_{0}$ limit.

\subsection{Results for the Imaginary Part in QCD}

In the large- $\beta_{0}$ limit, as discussed in Ch. 5 , the standard Padé approximants were not the best method to obtain reliable estimates for the higher-order coefficients and determine the characteristics of the perturbative series, however we will start with them for completeness.

At this point we will apply the Borel-Padé method to the perturbative series of the reduced imaginary part as a function of the scale-dependent mass and in the $\overline{\mathrm{MS}}$ scheme. The perturbative coefficients of this series are given in Eq. (3.55) and its singularities are expected to be at the same positions as the renormalons in the large- $\beta_{0}$ limit, ${ }^{9}$ i.e., at $u=-1,-2, \ldots$ and $u=3,4, \ldots$ There are only two non-trivial Padés to the Borel transform of this series that can be constructed using the first three known coefficients: $P_{1}^{1}$ and $P_{2}^{0}$, whose expressions are

$$
\begin{gathered}
P_{1}^{1}(u)=\frac{7.9106+35.022 u}{1.3960-u}, \\
P_{2}^{0}(u)=\frac{0.2488}{(0.1130+0.1765 i-u)(0.1130-0.1765 i-u)} .
\end{gathered}
$$


Table 4 - Coefficients $c_{n}$ in the $\overline{\mathrm{MS}}$ scheme predicted by the Padés to the Borel transform of the reduced imaginary part in QCD.

\begin{tabular}{ccccccc}
\hline & $c_{4}$ & $c_{5}$ & $c_{6}$ & $c_{7}$ & $c_{8}$ & $c_{9}$ \\
\hline$P_{1}^{1}(u)$ & 89.74 & 257 & 921 & 3958 & 19848 & 113742 \\
$P_{1}^{2}(u)$ & - & 21771 & -717557 & $2.84 \times 10^{7}$ & $-1.31 \times 10^{9}$ & $6.91 \times 10^{10}$ \\
$P_{2}^{1}(u)$ & - & -9319 & 11967 & $1.84 \times 10^{6}$ & $2.14 \times 10^{7}$ & $-3.01 \times 10^{8}$ \\
\hline
\end{tabular}

Source: By the author.

One can notice that $P_{2}^{0}$ has a pair of complex poles near the origin and, as we saw in large- $\beta_{0}$, the results of approximations that have poles of this type are not reliable. $P_{1}^{1}$ has an IR pole and its prediction for the fourth-order coefficient is not good: the error is around $110 \%$. However, the unsatisfactory results of this Padé were awaited since in large- $\beta_{0}$ the estimates using three coefficients as initial parameters were not trustworthy.

The three Padés that predicts the fifth-order coefficient: $P_{1}^{2}, P_{2}^{1}$ and $P_{3}^{0}$, are given by

$$
\begin{gathered}
P_{1}^{2}(u)=\frac{0.8597+10.089 u+32.314 u^{2}}{0.1517+u}, \\
P_{2}^{1}(u)=\frac{0.9317+3.0232 u}{(0.1561+0.3742 i-u)(0.1561-0.3742 i-u)}, \\
P_{3}^{0}(u)=\frac{0.0767}{(0.0302+0.2318 i-u)(0.0302-0.2318 i-u)(0.2477-u)} .
\end{gathered}
$$

Each PA has a pole in a different location: $P_{1}^{2}$ has an UV pole, $P_{3}^{0}$ has an IR one and $P_{2}^{1}$ a pair of complex poles, that is also present in $P_{3}^{0}$. The singularities of the Padés cannot be identified with any known renormalon of the series in QCD. This was expected because the function has branch cuts and, as we saw in Sec. 4.3, the Padé recreates the cuts by accumulating poles, which can only be observed for a great number of poles, i.e., for a PA of higher order. We can see that the complex poles of $P_{3}^{0}$ are very close to $u=0$, thus we will not consider its results.

These Padé approximants were expanded and their predictions for the coefficients $c_{n}$ of the imaginary part are in Tab. 4. The results are not stable, each Padé has a prediction with a different sign for the coefficients, which indicates that the estimates are not reliable. In spite of the bad predictions for higher-order coefficients, the Borel integral computed from these Padé approximants are stable. The Borel sum, which is determined as the mean value of the three Padés, is given by, for $\alpha_{s}\left(m_{H}\right)=0.1125$,

$$
F_{s}^{P}=0.241 \pm(0.019)_{\mathrm{PA}},
$$

where the uncertainty is the maximum spread found between two distinct predictions. We abstain from showing the Borel ambiguity because it is extremely small, of the order of 
$\mathscr{O}\left(10^{-14}\right)$. One can notice that the value found is greater than the large- $\beta_{0}$ one. This could be expected since the coefficients in QCD, at least until fourth order, are higher than the large- $\beta_{0}$ ones. The stable estimates for the Borel integral, even with extremely different predicted coefficients, can be explained by the very small value of the strong coupling at the Higgs mass, $\alpha_{s}\left(m_{H}\right)=0.1125$, which suppresses the coefficients.

Even though the D-log Padé was not impressive in the large- $\beta_{0}$ limit for the Borel transform of the imaginary part, we also tested it and the results are not different than the standard Padé ones: the predictions for the coefficients are not stable but the Borel integral is, and the value is close to the one found in Eq. (6.1). Hence we will not exhibit the results in detail.

The renormalization scheme was also changed as we did in large- $\beta_{0}$. In order to do that, we employ Eq. (2.74) into Eq. (3.54) that will be re-expanded in the new coupling $\tilde{a}_{s}$. For $C=-5 / 3$, the scheme we used in Ch. 5 , the reduced imaginary part is written as

$$
F^{(C=-5 / 3)}=5.6667 \tilde{a}_{s}+11.045 \tilde{a}_{s}^{2}-110.11 \tilde{a}_{s}^{3}-577.93 \tilde{a}_{s}^{4}+\ldots .
$$

We can notice that after changing to a less perturbative scheme (higher value of $\alpha_{s}$ ), where the UV poles dominate more, the sign-alternating behavior is not present, which indicates that the perturbative series in QCD is more dominated by an IR pole at intermediate orders, which does not happen in the large- $\beta_{0}$ limit. Padé approximants were calculated in this scheme $(C=-5 / 3)$ and in more perturbative schemes (where the IR poles dominate more), and no sign of improvement in the predictions could be observed, which is not surprising since there is no evidence that the UV poles dominate at intermediate orders. Due to this fact, we will only work in the $\overline{\mathrm{MS}}$ scheme from now on.

We also analyzed the Padé to the Borel transform of the imaginary part in terms of the invariant mass $\widehat{m}$, given in Eq. (3.56), since in the large- $\beta_{0}$ limit it gave the best results. The estimates are still unstable and consequently not trustworthy and no significant improvement could be noted.

\subsection{Results for the Second Derivative in QCD}

Since the Padés to the Borel transform of the imaginary part were not stable, we will at this point calculate Padés to the Borel transform of the reduced second derivative written in terms of the scale-dependent mass in the $\overline{\mathrm{MS}}$ scheme whose perturbative coefficients are in Eq. (3.59). Even though we recognize that this is not the ideal strategy for functions with cuts, we will present the results as we did for the imaginary part. The singularities of this series are expected to be at $u=-1,-2, \ldots$ and $u=2,3, \ldots$, the same position as

the renormalons in the large- $\beta_{0}$ limit. ${ }^{9}$ The two non-trivial Padés that we can construct with the first three coefficients are 
Table 5 - Coefficients $r_{n}$ in the $\overline{\mathrm{MS}}$ scheme predicted by the Padés to the Borel transform of the reduced second derivative in $\mathrm{QCD}$.

\begin{tabular}{ccccccc}
\hline & $r_{4}$ & $r_{5}$ & $r_{6}$ & $r_{7}$ & $r_{8}$ & $r_{9}$ \\
\hline$P_{1}^{1}(u)$ & 184 & 1143 & 8850 & 82240 & 891707 & $1.10 \times 10^{7}$ \\
$P_{2}^{0}(u)$ & -106 & -4720 & -68010 & -463649 & $8.07 \times 10^{6}$ & $4.02 \times 10^{8}$ \\
$P_{1}^{2}(u)$ & - & 796 & 5149 & 39954 & 361671 & $3.74 \times 10^{6}$ \\
$P_{2}^{1}(u)$ & - & 740 & 4297 & 29376 & 231963 & $2.08 \times 10^{6}$ \\
$P_{3}^{0}(u)$ & - & 2552 & 51825 & 784641 & $1.07 \times 10^{7}$ & $2.08 \times 10^{8}$ \\
\hline
\end{tabular}

Source: By the author.

$$
\begin{gathered}
P_{1}^{1}(u)=\frac{2.3672+4.6033 u}{0.6456-u} \\
P_{2}^{0}(u)=\frac{0.5397}{(0.2571+0.2848 i-u)(0.2571-0.2848 i-u)} .
\end{gathered}
$$

As in the imaginary part, $P_{1}^{1}$ has an IR pole and $P_{2}^{0}$ has a pair of complex poles. The predictions for the fourth-order coefficient, which is known, is accurate just for the first Padé: the error is around $20 \%$. For the other PA, $P_{2}^{0}$, is approximately $169 \%$, which is a large difference from the original value. A possible explanation for this bad estimate is because this Padé cannot freely alter the residue of its poles and also this PA has a pair of complex poles relatively close to the origin, which could worsen the predictions.

The Padés that estimate the fifth-order coefficient are given by

$$
\begin{gathered}
P_{1}^{2}(u)=\frac{2.8354+6.2390 u+2.5337 u^{2}}{0.7733-u}, \\
P_{2}^{1}(u)=\frac{5.1514+8.9679 u}{(0.8981-u)(1.5643-u)}, \\
P_{3}^{0}(u)=\frac{0.3100}{(0.0966+0.4609 i-u)(0.0966-0.4609 i-u)(0.3813-u)} .
\end{gathered}
$$

All the PAs have, at least, one IR pole close to $u=1$, except $P_{2}^{0}$, indicating that in QCD the second derivative is more dominated by the IR renormalons. Thus the perturbative series should be fixed-sign at intermediate orders, which is observed up to $\alpha_{s}^{4}$.

The coefficients $r_{n}$ of the second derivative predicted by the Padé approximants above are exhibited in Tab. 5. We can notice that the estimates of $P_{2}^{0}$ are very different when compared with the other Padé predictions and this occurred because of the poles of this Padé. In addition, the coefficients estimated by $P_{1}^{1}, P_{1}^{2}, P_{2}^{1}$ and $P_{3}^{0}$ are all positive, corroborating that IR poles dominate the perturbative series at intermediate orders. The coefficients estimated by $P_{3}^{0}$ are larger than the other ones which could be explained by the proximity of its complex poles to the origin. As we saw in large- $\beta_{0}$, these poles spoil 
the convergence of the Padé.

It is known that the renormalons of the Borel transform in QCD are in the same locations as in large- $\beta_{0}$, however, instead of poles, they are branch points. ${ }^{9}$ Thus we know that the closest renormalon to the origin is an UV one, $u=-1$, which will govern the behavior of the series at higher orders. Since the predicted coefficients are not signalternating, this implies that an IR renormalon is more dominant at intermediate orders and a possible reason for this is a larger residue for the IR renormalon. Hence, with only the coefficients we have, the Padé is unable to "see" the effects of the UV pole.

We also tested Padé approximants to the Borel transform of the reduced second derivative when the perturbative series is written in terms of the invariant mass $\widehat{m}$, as in Eq. (3.60). However the results did not improve when compared to the ones in Tab. 5.

\subsubsection{D-log Padés to the Second Derivative in QCD}

We will at this point construct D-log Padés to the Borel transform of the reduced second derivative in the $\overline{\mathrm{MS}}$ scheme. We expect that the results of the D-log Padés improve compared to the standard Padés to the imaginary part and the second derivative, since this approximant is appropriated for functions with branch cuts and also was the best strategy in the large- $\beta_{0}$ limit.

The following D-log Padés were calculated: $\operatorname{Dlog}_{0}^{1}$ and $\operatorname{Dlog}_{1}^{0}$, that estimate the fourthorder coefficient, $\operatorname{Dlog}_{1}^{1}, \operatorname{Dlog}_{2}^{0}$ and $\operatorname{Dlog}_{0}^{2}$, which use the coefficient $r_{4}$ as input, and their expressions are shown below

$$
\begin{gathered}
\operatorname{Dlog}_{1}^{0}(u)=0.00102(2.5275+u)^{8.8299}, \\
\operatorname{Dlog}_{0}^{1}(u)=3.6667 \mathrm{e}^{\left(3.4936 u-0.6911 u^{2}\right)}, \\
\operatorname{Dlog}_{2}^{0}(u)=5.3279 \frac{(0.6399+u)^{1.2799}}{(0.8569-u)^{1.2799}}, \\
\operatorname{D}_{\log _{0}^{2}}(u)=3.6667 \mathrm{e}^{\left(3.4936 u-0.6911 u^{2}+2.3059 u^{3}\right)} .
\end{gathered}
$$

We did not show the expression of $\operatorname{Dlog}_{1}^{1}$ because the Padé $P_{1}^{1}$ used to build this D-log has a defect: a pole at $u=-0.1998$ and a close-by zero at $u=-0.2170$, which, as we saw in Ch. 5, reduces the order of the PA and produces untrustworthy estimates. Regarding the renormalons, only $\operatorname{Dlog}_{2}^{0}$ predicts a cut on the positive real axis at $u=0.8569$ with multiplicity $\gamma=1.2799$, close to the poles of the standard Padés.

The D-log Padés were expanded and their predicted coefficients are shown in Tab. 6. As we can see, the values estimated by $\operatorname{Dlog}_{0}^{1}$ and $\operatorname{Dlog}_{1}^{0}$ for the coefficient of $a_{s}^{4}$ are close to the real one, which is 154: the error is of approximately 30\%. Even though the predicted values of $r_{4}$ are satisfactory, the higher-order coefficients are very different from the ones obtained by other D-log Padés and by the standard Padés. This can be explained by the 
Table 6 - Coefficients $r_{n}$ in the $\overline{\mathrm{MS}}$ scheme predicted by the D-log Padés to the Borel transform of the reduced second derivative in QCD.

\begin{tabular}{lcccccc}
\hline & $r_{4}$ & $r_{5}$ & $r_{6}$ & $r_{7}$ & $r_{8}$ & $r_{9}$ \\
\hline $\operatorname{Dlog}_{1}^{0}(u)$ & 107 & 247 & 473 & 716 & 802 & 581 \\
$\operatorname{Dlog}_{0}^{1}(u)$ & 103 & 196 & 114 & -956 & -4287 & -5728 \\
$\operatorname{Dlog}_{2}^{0}(u)$ & - & 789 & 4877 & 36178 & 307326 & $2.99 \times 10^{6}$ \\
$\operatorname{Dlog}_{0}^{2}(u)$ & - & 905 & 5605 & 34626 & 262295 & $2.21 \times 10^{6}$ \\
\hline
\end{tabular}

Source: By the author.

fact that not all functions will be well approximated by a straight line or a monopole, the PAs used to build these D-log Padés. In addition, in the large- $\beta_{0}$ limit the second derivative was not accurately replicated by these approximants, which is an indication that it would not work properly in QCD.

Except for Dlog${ }_{0}^{1}$, all the approximants predict positive coefficients, as do the standard Padés. Furthermore, $\operatorname{Dlog}_{2}^{0}$ and $\operatorname{Dlog}_{0}^{2}$ estimate values closer to the ones already obtained from the PAs. The fixed-sign behavior and the IR singularities corroborate that the IR renormalons govern the behavior of the series at lower and intermediate orders.

\subsubsection{Padés to the Series in $a_{s}$ in QCD}

A possible way to corroborate the results we found previously is to study the series of the second derivative in $a_{s}$. This result is less interesting because we lose the connection with the renormalons and our knowledge of the Borel transform, however it can be useful in this sense to corroborate what we have done so far. One can also notice from Eq. (3.59) that the perturbative series of the second derivative in QCD is very regular until fourth order, i.e., there is no change of sign and the known coefficients are stable (the divergent behavior is not evident up to fourth-order). Hence it is likely that the Padé approximants to the series in $a_{s}$ should work properly. ${ }^{18}$ We then applied Padés that uses three and four coefficients as inputs $\left(P_{2}^{1}, P_{1}^{2}, P_{3}^{1}, P_{1}^{3}\right.$ and $\left.P_{2}^{2}\right)$ to the series in $a_{s}$ of the reduced second derivative in the $\overline{\mathrm{MS}}$ scheme.

The Padé approximants were expanded and the coefficients $r_{n}$ obtained from them are in Tab. 7. The results for $P_{2}^{2}$ are not in the table because it has a defect, a pole at $u=-0.1568$ and a zero at $u=-0.1535$. This almost cancellation between the pole and the zero reduces the PA order and the predictions are not reliable.

The predictions for the fourth-order coefficient $r_{4}$ by the Padés $P_{1}^{2}$ and $P_{2}^{1}$ are in good agreement with the real value, with an error of approximately $20 \%$. Analyzing the coefficients estimated in Tab. 7 we can see that the results are very stable and all the coefficients are positive, as the ones found for the standard Padés and the D-log Padés to the second derivative. This suggests that the IR renormalons are indeed dominant at 
Table 7 - Coefficients $r_{n}$ in the $\overline{\mathrm{MS}}$ scheme predicted by the Padés to the perturbative series in $a_{s}$ of the reduced second derivative in QCD.

\begin{tabular}{ccccccc}
\hline & $r_{4}$ & $r_{5}$ & $r_{6}$ & $r_{7}$ & $r_{8}$ & $r_{9}$ \\
\hline$P_{1}^{2}\left(a_{s}\right)$ & 123 & 381 & 1180 & 3655 & 11323 & 35079 \\
$P_{2}^{1}\left(a_{s}\right)$ & 121 & 368 & 1117 & 3395 & 10316 & 31347 \\
$P_{1}^{3}\left(a_{s}\right)$ & - & 597 & 2317 & 8990 & 34875 & 135301 \\
$P_{3}^{1}\left(a_{s}\right)$ & - & 598 & 2235 & 8368 & 31533 & 118727 \\
\hline
\end{tabular}

Source: By the author.

lower and intermediate orders. However, the values of the coefficients predicted in Tab. 7 are lower than the ones obtained before.

\subsection{Final Results}

At this section we will calculate the higher-order coefficients of the perturbative series of the imaginary part. In order to do that, we will use the results in QCD that showed stability: the standard Padés to the Borel transform as well as to the series in $\alpha_{s}$ of the second derivative, whose results are in Tabs. 5 and 7 respectively, and the D-log Padés to the Borel transform of the second derivative, whose predictions are in Tab. 6. We will not use the Padés and D-log Padés that predict the third-order coefficient since in the large- $\beta_{0}$ limit they did not have enough information to obtain trustworthy predictions. We will also disregard the Padé $P_{3}^{0}(u)$ since it has complex poles near the origin.

First of all, as we did in Sec. 5.4, we will compute the independent coefficients $d_{n, 1}$. In order to extract them from the fifth- to the eighth-order, in principle we need the coefficients of the $\beta$ and $\gamma$ functions up to seventh- and eighth-order respectively. Since we know exactly only the coefficients up to fifth-order, we will consider the unknown higherorder terms of the $\beta$ and $\gamma$ functions equal to zero, i.e., $\beta_{6}=\beta_{7}=0$ and $\gamma_{6}=\gamma_{7}=\gamma_{8}=0$. For $N_{f}=5$, the known coefficients of these two functions in terms of the coupling $\alpha_{s}$ are

$$
\begin{aligned}
& \frac{\beta\left(\alpha_{s}\right)}{\beta_{1} \alpha_{s}^{2}}=1+0.4013 \alpha_{s}+0.1494 \alpha_{s}^{2}+0.3172 \alpha_{s}^{3}+0.0809 \alpha_{s}^{4}+\ldots, \\
& \frac{\gamma\left(\alpha_{s}\right)}{\gamma_{1} \alpha_{s}}=1+1.1185 \alpha_{s}+0.7518 \alpha_{s}^{2}+0.3559 \alpha_{s}^{3}+0.4293 \alpha_{s}^{4}+\ldots,
\end{aligned}
$$

and for $N_{f}=6$

$$
\begin{aligned}
& \frac{\beta\left(\alpha_{s}\right)}{\beta_{1} \alpha_{s}^{2}}=1+0.2956 \alpha_{s}-0.0294 \alpha_{s}^{2}+0.1780 \alpha_{s}^{3}+0.0016 \alpha_{s}^{4}+\ldots \\
& \frac{\gamma\left(\alpha_{s}\right)}{\gamma_{1} \alpha_{s}}=1+1.0743 \alpha_{s}+0.4903 \alpha_{s}^{2}-0.1454 \alpha_{s}^{3}-0.1002 \alpha_{s}^{4}+\ldots
\end{aligned}
$$

which does not show any sign of a possible divergence for these perturbative series. ${ }^{36,37,61}$ 
Table 8 - Coefficients $d_{n, 1}$ in the $\overline{\mathrm{MS}}$ scheme predicted by Padés and D-log Padés to the second derivative in QCD.

\begin{tabular}{ccccc}
\hline & $d_{5,1}$ & $d_{6,1}$ & $d_{7,1}$ & $d_{8,1}$ \\
\hline$P_{1}^{2}(u)$ & 40968 & 549637 & $8.37 \times 10^{6}$ & $1.43 \times 10^{8}$ \\
$P_{2}^{1}(u)$ & 40912 & 548137 & $8.34 \times 10^{6}$ & $1.42 \times 10^{8}$ \\
$\operatorname{Dlog}_{2}^{0}(u)$ & 40961 & 549284 & $8.36 \times 10^{6}$ & $1.43 \times 10^{8}$ \\
$\operatorname{Dlog}_{0}^{2}(u)$ & 41077 & 551531 & $8.39 \times 10^{6}$ & $1.43 \times 10^{8}$ \\
$P_{1}^{3}\left(a_{s}\right)$ & 40769 & 544499 & $8.26 \times 10^{6}$ & $1.41 \times 10^{8}$ \\
$P_{3}^{1}\left(a_{s}\right)$ & 40770 & 544430 & $8.26 \times 10^{6}$ & $1.41 \times 10^{8}$ \\
\hline
\end{tabular}

Source: By the author.

Table 9 - Final values for the coefficients $d_{n, 1}$ in $\overline{\mathrm{MS}}$ obtained from the Padé approximants of Tab. 8 in QCD.

\begin{tabular}{cccc}
\hline$d_{5,1}$ & $d_{6,1}$ & $d_{7,1}$ & $d_{8,1}$ \\
\hline$(4.09 \pm 0.03) \times 10^{4}$ & $(5.48 \pm 0.07) \times 10^{5}$ & $(8.33 \pm 0.13) \times 10^{6}$ & $(1.42 \pm 0.02) \times 10^{8}$ \\
\hline
\end{tabular}

Source: By the author.

In addition, it is believed that the $\overline{\mathrm{MS}}$ scheme is a regular scheme, i.e., a scheme where the $\beta$ and $\gamma$ functions are convergent series or at least do not diverge as fast as a factorial ${ }^{9}$ however there is no proof of this conjecture. As a check of the stability of this procedure, we also computed the coefficients $d_{5,1}$ zeroing the last known coefficient, $\beta_{5}$ and $\gamma_{5}$, and compared the results found using $\gamma_{5}$ of Eq. (2.60) and $\beta_{5}$ of Eq. (2.64). The difference did not exceed $0.5 \%$, which confirms that the truncation of the $\beta$ and $\gamma$ function at sixth-order is a good approximation. This agrees with the expectation that the $\overline{\mathrm{MS}}$ is a regular scheme. This assumption will be used in the rest of this work.

The coefficients $d_{n, 1}$ were calculated from the estimated values of $r_{n}$ using Eq. (5.48) and the results found are in Tab. 8. As one can notice, the results are very stable, much more than the value for the coefficient $r_{n}$ indicated in Tabs. 5, 6 and 7. This was already observed in large- $\beta_{0}$ and it occurs because the coefficients $r_{n}$ depend on $d_{n, 1}$, hence the errors associated with $r_{n}$ should be larger. The final values for the coefficients $d_{n, 1}$ are indicated in Tab. 9 and were calculated as the average between the Padés exhibited in Tab. 8. The errors are equal to the maximum spread found between two PAs.

With the results of Tab. 9 it is possible to calculate the higher-order coefficients $c_{n}$ of the imaginary part in terms of the scale-dependent mass $m(\mu)$. Let us calculate as an example the coefficient $c_{5}$. From (5.45) we have

$$
c_{5}=d_{5,1}-\pi^{2} d_{5,3}+\pi^{4} d_{5,5} .
$$

Employing now the relations between the coefficients $d_{n, k}$ and $d_{n, 1}$ exhibited in Eq. (5.47) and the values for the coefficients $d_{n, 1}$, the estimate for $c_{5}$ is 
Table 10 - Final values for the coefficients $c_{n}$ of the imaginary part of the scalar correlator in QCD obtained from the results in Tab. 9.

\begin{tabular}{cccc}
\hline$c_{5}$ & $c_{6}$ & $c_{7}$ & $c_{8}$ \\
\hline$-8200 \pm 308$ & $(-2.80 \pm 0.69) \times 10^{4}$ & $(1.48 \pm 2.05) \times 10^{5}$ & $(2.39 \pm 4.97) \times 10^{6}$ \\
\hline
\end{tabular}

Source: By the author.

$$
c_{5}^{P}=-8200 \pm 308
$$

And for the next order, our estimate is

$$
c_{6}^{P}=-(2.80 \pm 0.69) \times 10^{4} .
$$

We can compare our result for the fifth-order coefficient with other estimates in the literature. This comparison is not completely straightforward since these other estimates do not have associated errors reported, thus we can only assume that those values are exact. The first method, applied by Bakulev, Mikhailov and Stefanis, ${ }^{62,63}$ models the coefficients of the series with two parameters, which are determined through the known coefficients of the series. Their central value for the coefficient of $a_{s}^{5}$ is approximately half of the one obtained by us, and the results are not compatible if we consider our uncertainty. They also calculated this coefficient using the strategy employed by Kataev and Starshenki, ${ }^{64}$ the Principle of Minimal Sensitivity (PMS), and the value obtained, which is around -6886 , is not compatible to our prediction as well.

The predictions for the other coefficients are shown in Tab. 10 together with their uncertainties. One can notice that the errors are higher than the ones of the coefficients $d_{n, 1}$. This occurred because, as we discussed in the large- $\beta_{0}$ case, the coefficients $c_{n}$ depend on $d_{n, k}$ that are proportional to $d_{n, 1}$, which causes the increase in the uncertainties. For the coefficients of seventh-order or higher, the errors associated are greater than $100 \%$. However, the value of the strong coupling $\alpha_{s}$ suppresses these errors generating a not so large uncertainty in the sum of the series. It is also possible to see that the estimated coefficients $c_{n}$ are not sign-alternated, which suggests that IR renormalons dominate more than UV ones at lower and intermediate orders as in the second derivative case. A possible reason for this behavior is that the residue of the UV pole is smaller than the IR one.

In addition, we can also compute the Borel integral for the imaginary part using the same Padé approximants employed to determine the independent coefficients $d_{n, 1}$. In order to do that, we calculated the perturbative series of the imaginary part predicted by each Padé. With those series we were able to determine the perturbative expansion of the Borel transform of the imaginary part and finally the Borel integral. In the interest of checking the stability of this procedure, we computed the Borel integral of the second derivative using the expressions of the Padés given in Sec. 6.2 and also using the expansion 
of these PAs. The results found differ in the sixth decimal place, showing that this method is trustworthy. Hence, for $\alpha_{s}\left(m_{H}\right)=0.1125 \pm 0.0010$, the mean value obtained for the reduced imaginary part is

$$
F^{P}=0.24034 \pm(0.02471)_{\alpha_{s}} \pm(0.00003)_{\mathrm{PA}},
$$

where the first error is due to the uncertainty of the coupling constant and the second one is the maximum spread found between two Padé approximants. Since we obtain the Borel sum through the perturbative series of the Borel transform, there is no Borel ambiguity in our final result. We expect that the Borel ambiguity would be very small because in large- $\beta_{0}$ these uncertainties were extremely low and, as we can see in Eq. (6.1), which gives the estimate from the Padés to the imaginary part, the ambiguity due to the poles on the positive real axis is much smaller than the difference between the predictions. We can see that the dominant term for the integral error is the uncertainty of the strong coupling. Our result is in good agreement with the one determined through the Principle of Maximum Conformality (PCM), ${ }^{65,66}$ which obtained $0.2405 \pm 0.0001$.

We are also able to finally write the decay width of the Higgs boson into bottom quarks up to fifth order in the Higgs rest frame as

$$
\begin{aligned}
\Gamma(H \rightarrow b \bar{b})=\frac{N_{c}}{8 \pi v^{2}} m_{b}^{2} m_{H}[1+5.6667 & a_{s}+29.1467 a_{s}^{2}+41.7576 a_{s}^{3}+ \\
& \left.-825.747 a_{s}^{4}-(8200 \pm 308) a_{s}^{5}+\mathscr{O}\left(a_{s}^{6}\right)\right] .
\end{aligned}
$$

We can calculate numerically the corrections to the decay rate up to fifth-order at the scale $\mu=m_{H}$, which is given below

$$
\Gamma(H \rightarrow b \bar{b})=\frac{N_{c}}{8 \pi v^{2}} m_{b}^{2} m_{H}[1+0.2029+0.0374+0.0019-0.0014-0.0005] .
$$

As we can notice, the correction we are estimating, the fifth-order one, is indeed very small compared to the others: it is less than a half of the fourth-order correction.

We can use the result of Eq. (6.10) to study the decay rate dependence on the renormalization scale $\mu$ order by order. First one can write the decay width at the Higgs rest frame as

$$
\Gamma(H \rightarrow b \bar{b})=\Gamma^{\mathrm{LO}}\left(1+\sum_{n=1}^{\infty} a_{\mu}^{n} \Gamma^{(n)}\right)=\frac{N_{c}}{8 \pi v^{2}} m_{b}^{2} m_{H}\left(1+\sum_{n=1}^{\infty} a_{\mu}^{n} \Gamma^{(n)}\right) .
$$

The terms $\Gamma^{(n)}$ depend on the renormalization scale, and in order to compute them, one has to reconstruct all the logarithms that were summed when we chose $\mu^{2}=s=m_{H}^{2}$. This can be easily done if we remind that the imaginary part of the scalar correlator satisfies a homogeneous RGE. Calculating the logarithms, disregarding for the moment the uncertainty of the fifth-order coefficient, one finds for $N_{f}=5$ up to fifth-order 


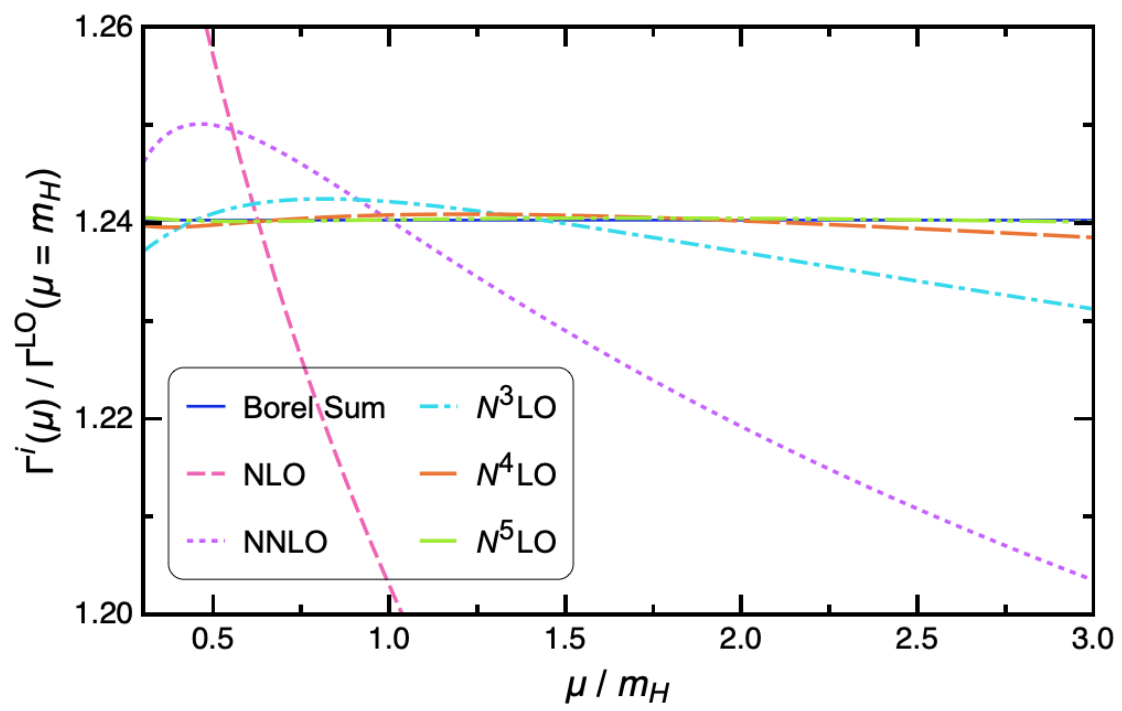

Figure 39 - Dependence on the Higgs decay rate with the renormalization scale $\mu$.

Source: By the author.

$$
\begin{aligned}
& \Gamma^{(1)}=5.6667-2 L, \\
& \Gamma^{(2)}=29.147-29.222 L+3.9167 L^{2}, \\
& \Gamma^{(3)}=41.758-238.38 L+94.676 L^{2}-7.6157 L^{3}, \\
& \Gamma^{(4)}=-825.75-791.51 L+1115 L^{2}-260.065 L^{3}+14.756 L^{4}, \\
& \Gamma^{(5)}=-8200+6473 L+5868 L^{2}-4069 L^{3}+655.67 L^{4}-28.527 L^{5},
\end{aligned}
$$

where $L=\ln \left(m_{H}^{2} / \mu^{2}\right)$. With these terms, we plot the ratio $\Gamma^{i} / \Gamma^{L O}\left(\mu=m_{H}\right)$, with the denominator calculated at $\mu=m_{H}$ and $i=\left\{\mathrm{NLO}, \mathrm{NNLO}, \mathrm{N}^{3} \mathrm{LO}, \mathrm{N}^{4} \mathrm{LO}, \mathrm{N}^{5} \mathrm{LO}\right\}$, as a function of $\mu / m_{H}$, which was varied in a very wide range from 0.3 to 3 , and the final result is in Fig. 39. The solid blue line is the central value of the Borel integral given in Eq. (6.9) plus the leading order correction. The values for the strong coupling $\alpha_{s}$ and the bottom-quark mass $m_{b}(\mu)$ at different renormalization scales were obtained using RunDec. ${ }^{67}$ It is possible to notice that the $\mathrm{N}^{4} \mathrm{LO}$ term is already in agreement with the value of the Borel integral at the range of Fig. 39, indicating that the decay rate has a small dependence on the renormalization scale from the fourth-order correction. We can compare our plot in Fig. 39 with the one in the paper of Mondini, Schiavi and Williams, ${ }^{68}$ and it is possible to note that up to next to leading order (NLO) our result is very similar to theirs ${ }^{\dagger \dagger}$. We can observe that the lower-order terms depend more on the renormalization scale than the higher ones. In other words, when higher-order corrections are included, the decay width stabilizes, which was already expected.

In order to analyze the higher-order contributions at different scales, we plotted, in ${ }^{\dagger}$ Our result is more stable than theirs for NNLO and higher orders. 


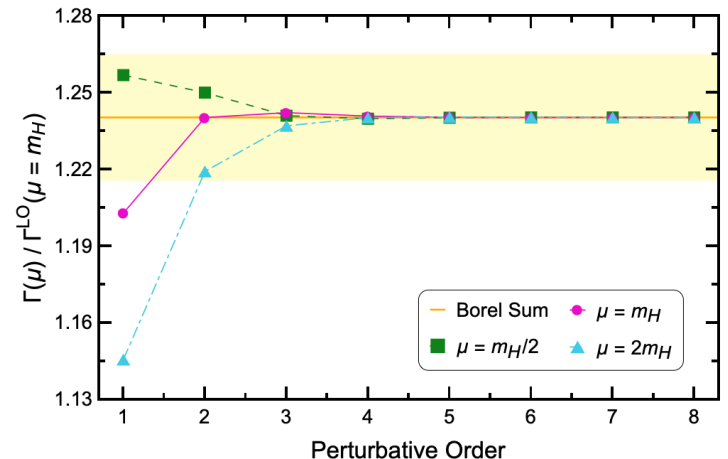

(a)

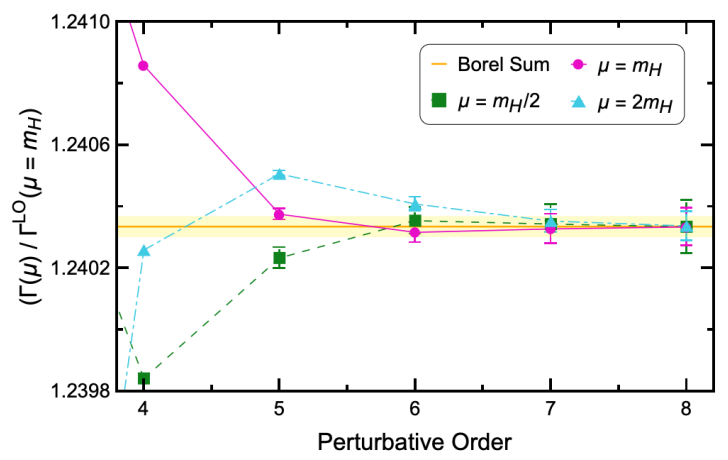

(b)

Figure 40 - Perturbative expansion of the decay rate in QCD at different renormalization scales using the coefficients of Tab. 10 and the integral given in Eq. (6.9). The uncertainty of the Borel integral is due to (a) the strong coupling uncertainty and (b) the different Padé predictions. The uncertainty bars of the points in (b) are due to the errors of the predicted coefficients.

Source: By the author.

Figs. 40a and 40b, the perturbative expansion of the decay rate in QCD at a scale $\mu$ divided by the leading order term at the scale $\mu=m_{H}$, and the renormalization scales employed were $\mu=\frac{m_{H}}{2}, m_{H}, 2 m_{H}$. The error of the Borel integral is, for Fig. 40a, due to the strong coupling uncertainty and, for Fig. 40b, only due to the different Padé estimates. The errors at each order of the decay width are not as large as the ones in Tab. 10 because the strong coupling is small at these scales, which suppresses the errors of the coefficients. One can see that all the results are compatible with the Borel integral calculated by us at higher-orders, corroborating that the imaginary part of the scalar correlator has a very small dependence on the renormalization scale. In addition, for the three scales used, the fifth-order correction is very small, indicating that we are close to the limit of what can be done with perturbative QCD in this decay, although this correction is necessary to stabilize the series and make it compatible with the Borel integral. We can also notice that the major limiting factors for the decay of the Higgs into $b$ quarks are the mass corrections and the precision of the strong coupling, the $b$-quark mass as well as the Higgs mass. 


\section{CONCLUSION}

In this dissertation we studied the Padé approximants to the Borel transform of the perturbative series in QCD of the Higgs decay into a pair of bottom quarks. The decay rate is associated with the imaginary part of the scalar correlator through the Optical Theorem by Eq. (3.16). Before we determined the results in QCD, we applied this method in the large- $\beta_{0}$ limit, that contains the highest-order term of $N_{f}$ for each power of the strong coupling $\alpha_{s}$ of QCD. In this limit the perturbative series is know to all orders, which allows us to analyze the quality of our predictions.

In Ch. 5 we presented and discussed the results obtained in the large- $\beta_{0}$ limit. First, we applied the Padé approximants to the Borel transform of the reduced imaginary part of the scalar correlator, which contains only the non-trivial terms of the perturbative series, written as a function of the scale-dependent quark mass in the $\overline{\mathrm{MS}}$ scheme. We could observe the convergence to the original series when the order of the Padé is raised in Figs. 27 and 28 as stated by Pomerenke's theorem. However some Padé approximants give bad results, and, in a few cases, we were able to justify it by the appearance of the defects, poles almost canceled by near-by zeros that effectively reduce the order of the Padé. Other type of Padés that should be discarded are the ones with poles distant from the origin. These Padés, when expanded around $u=0$, do not provide trustworthy results, since the denominator is very large. We also noted that, with seven or eight coefficients, the Padés could reproduce the main characteristics of the original function: the sign-alternation, the higher-order coefficients, the Borel integral, the dominant renormalon and its residue.

However, since the results considering only the first four coefficients, which is what is known in QCD, were not exceptional, other strategies had to be investigated. The first one was to change the renormalization scheme of the perturbative series written in terms of the invariant quark mass using negative values of $C$. Schemes with $C$ negative improved a lot the results as we can see in Fig. 29, which clearly shows the acceleration of the convergence. The errors are now approximately one order of magnitude lower, and even after being brought back to the $\overline{\mathrm{MS}}$ scheme the results are better, as indicated in Tab. 1. This occurred because the Borel transform in these schemes is highly dominated by the first UV pole, thus the sign-alternation can be observed earlier in the perturbative series, and the exponential term is canceled for $C=-5 / 3$; so the Padés can approximate easier this function.

We also calculated the partial Padé approximants, that use the knowledge about the analytical structure of the series. Adding the first two dominant renormalons of the Borel transform to the partial Padés improves the results since most of the PPAs reproduce the 
sign-alternation, which was not be observed in the standard PAs. However, the errors are considerably lower only at higher orders. When the scheme was changed, the errors were one to two orders smaller than the PPAs in the usual scheme, the $\overline{\mathrm{MS}}$. When compared to the standard Padés in the same scheme, where $C=-5 / 3$, the results are not significantly better for Padés that uses few coefficients as input, although the errors are smaller at higher values of $N$. A reason for the little improvement is that the series of the imaginary part is mostly dominated by the no-pole term at lower orders, the first UV renormalon $u=-1$ only starts to dominate in the seventh-order coefficient.

Due to the not so impressive results using just four coefficients, the Padé-Borel method was also applied to the reduced second derivative written as a function of the invariant mass $\widehat{m}$. The standard Padés got worse results even when the scheme was modified to $C=-5 / 3$, as illustrated in Tab. 3, because the Borel transform of this series has a lot of double poles, hence the Padé spends more coefficients "trying" to reproduce them.

We also analyzed the D-log Padés to this series in the $\overline{\mathrm{MS}}$ scheme and the results are awesome: the errors are very low, they mimic the leading renormalon of the Borel transform and the sign-alternation when the order of the D-log is raised. Changing scheme betters the estimates for the coefficients, as we can see in Fig. 38, as well as the dominant pole prediction, since now even its multiplicity is replicated. The D-log Padés had good results because the new function $F(z)$ turns the double poles of the Borel transform to single poles, which facilitates for the Padé into approximate.

Considering the D-log Padés that could be used in QCD, just Dlog ${ }_{1}^{1}$ and $\operatorname{Dlog}_{0}^{2}$ improved the estimates of the standard PAs. The D-log Padés that uses three coefficients as initial parameters, $D \log _{1}^{0}$ and $D \log _{0}^{1}$, got terrible results and this can be explained by the type of the PAs used to built them: not every function will be adequately approximated by $P_{1}^{0}$ or $P_{0}^{1}$, which is the case for the second derivative. For $\operatorname{Dlog}_{2}^{0}$, the problem is that the standard Padé $P_{2}^{0}$ used to construct it has a pair of complex poles very close to the origin, then its approximation to the function around $u=0$ cannot be used.

Hence, summing up, the most efficient strategy in the large- $\beta_{0}$ limit is the D-log Padés to the second derivative. Applying standard Padés to the imaginary part varying the renormalization scheme to less perturbative ones also worked well. The series at lower orders is not sufficiently regular for the PAs to approximate well, i.e., four coefficients as input is the minimum necessary to generate a good reproduction. Thus the Padé approximants that predicts the fourth-order coefficient are not accurate, they do not have sufficient information to obtain reliable estimates.

With these results in large- $\beta_{0}$, we turned to the analysis of the perturbative series in QCD in Ch. 6. First, we calculated standard Padés to the Borel transform of the reduced imaginary part written as a function of the scale-dependent quark mass. After disconsidering the Padés with complex poles near the origin, we could not find stable results for the predicted coefficients, as indicated in Tab. 4, not even when the scheme was 
modified or the perturbative series was written in terms of the invariant quark mass. When the renormalization scheme was changed to a less perturbative one, it was not possible to see a higher dominance of the UV renormalons, which indicates that IR poles govern the behavior of the imaginary part at lower and intermediate orders.

Later we calculated Padés and D-log Padés to the Borel transform of the reduced second derivative. We could notice that the estimated coefficients for the second derivative were positive and the Padés had poles on the positive real axis of $u$, which indicates that this series is more dominated by IR poles at intermediate orders, which is different from what we observed in large- $\beta_{0}$.

We also applied standard Padés to the series in $a_{s}$ of the second derivative in order to corroborate the results found so far. The stability of the known coefficients of this series (as we can see in Eq. (3.59), the coefficients change by a factor close to three) and the lack of sign change indicate that the Padés applied to it should work well. ${ }^{18}$ Then, the higher-order coefficients predicted by the PAs are all positive and the values are similar, though smaller, to the ones calculated previously.

To determine our estimate for the fifth-order coefficient of the imaginary part of the scalar correlator in QCD, we used the predictions of the standard Padés and the D-log Padés applied to the Borel transform of the second derivative and the Padés to the series in $a_{s}$ of the second derivative. However we did not use the Padé approximants that estimate the fourth-order coefficient since our experience in large- $\beta_{0}$ suggests that series with only three coefficients as input are not satisfactory. Our estimate is model independent because it only uses the known coefficients and the Padés employed have little or no information about the structure of the function. Our prediction for the fifth-order coefficient is then

$$
c_{5}^{P}=-8200 \pm 308 \text {. }
$$

Our result was compared to two other estimates found in the literature: the first one parametrizes the coefficients of the series with two parameters that are determined using the known coefficients of the series. ${ }^{62,63}$ Their prediction, $c_{5}=-4052$, is not compatible to our estimate. The second method, the PMS, that estimated a value of $-6886,{ }^{64}$ is also not in agreement with our result. None of these values on the literature has an associated error, hence we are forced to assume that they are exact.

Since we know that the singularities of the perturbative series in QCD are at the same location of the renormalons in the large- $\beta_{0}$ limit, ${ }^{9}$ the pole that dominates at higher orders is the leading UV pole at $u=-1$. Because we could not see a sign-alternation in the predicted coefficients shown in Tab. 10, this suggests that the perturbative series of the imaginary part is more dominated by IR renormalons at lower and intermediate orders, due to possibly their greater residue compared to the residue of UV poles. Another possible explanation for the sign behavior is a competition between different renormalons that leads to the UV contribution being deleted or hidden. 
We also estimate the Borel integral using the same Padés to the second derivative employed to predict the higher-order coefficients. Our result is $0.24034 \pm 0.02471 \pm 0.00003$, where the first error is due to the strong coupling uncertainty and the second one is the maximum difference between two Padé predictions. Our estimate is equivalent to the other value on the literature that is determined based on the PCM. ${ }^{65,66}$

We plotted the decay rate of the Higgs boson into bottom quarks order by order as a function of the renormalization scale $\mu$, which can be seen in Fig. 39. Our result up to NLO agrees with the literature, ${ }^{68}$ and we could conclude that the decay width dependence on the renormalization scale gets smaller when higher-order corrections are included, with a very modest dependence already at $\mathrm{N}^{4} \mathrm{LO}$. We could notice that for the interval $[0.3,3] m_{H}$ the decay rate at $\mathrm{N}^{4} \mathrm{LO}$ is compatible with the value of the Borel integral computed by us, showing that the dependence on the renormalization scale is very small in this range.

The perturbative expansion of the imaginary part was plotted for $\mu=m_{H} / 2$ and $\mu=2 m_{H}$ in Figs. 40a and 40b, and the results are in agreement with our value for the Borel integral. We could also note that although the fifth-order correction is rather small compared to the others for the three scales used $\left(\frac{m_{H}}{2}, m_{H}, 2 m_{H}\right)$, it is necessary to stabilize the series of the decay rate. Thus, this implies that the higher-order terms for this decay are no longer the limiting factors, which now are the precision of the bottom-quark and the Higgs masses, the mass corrections and mostly the accuracy of the strong coupling. As we already discussed, $m_{b}^{2} / m_{H}^{2}$ is of the order of $\mathscr{O}\left(10^{-3}\right)$, as well as the relative uncertainty of the Higgs mass, ${ }^{43}$ which is higher than our estimate for the fifth-order correction. The relative uncertainties of the bottom-quark mass and the strong coupling $\alpha_{s}$ are even larger: ${ }^{43}$ of the order of $\mathscr{O}\left(10^{-2}\right)$. It is safe to conclude that, at present, the limiting factor in the precision of the SM calculation of $\Gamma(H \rightarrow b \bar{b})$ is the uncertainty in the fundamental QCD parameters, namely $\alpha_{s}$ and $m_{b}$. 


\section{REFERENCES}

1 ENGLERT, F.; BROUT, R. Broken symmetry and the mass of gauge vector mesons. Physical Review Letters, v. 13, n. 9, p. 321-323, 1964.

2 HIGGS, P. W. Broken symmetries and the masses of gauge bosons. Physical Review Letters, v. 13, n. 16, p. 508-509, 1964.

3 AAD, G. et al. Observation of a new particle in the search for the Standard Model Higgs boson with the ATLAS detector at the LHC. Physics Letters B, v. 716, n. 1, p. $1-29,2012$.

4 CHATRCHYAN, S. et al. Observation of a new boson at a mass of $125 \mathrm{GeV}$ with the CMS experiment at the LHC. Physics Letters B, v. 716, n. 1, p. 30-61, 2012.

5 SIRUNYAN, A. M. et al. Combined measurements of Higgs boson couplings in proton-proton collisions at $\sqrt{s}=13 \mathrm{TeV}$. The European Physical Journal C, v. 79, n. 5 , p. $421,2019$.

6 BAIKOV, P.; CHETYRKIN, K.; KÜHN, J. Scalar correlator at $\mathscr{O}\left(\alpha_{s}^{4}\right)$, Higgs decay into bottom quarks and bounds on the light-quark masses. Physical Review Letters, v. 96, n. 1, p. 012003, 2006.

7 HERZOG, F. et al. On Higgs decays to hadrons and the R-ratio at $\mathrm{N}^{4}$ LO. Journal of High Energy Physics, v. 2017, n. 8, p. 113, 2017.

8 CHETYRKIN, K. G. Correlator of the quark scalar currents and $\Gamma_{\text {tot }}(H \rightarrow$ hadrons $)$ at $O\left(\alpha_{s}^{3}\right)$ in pQCD. Physics Letters B, v. 390, n. 1, p. 309-317, 1997.

9 BENEKE, M. Renormalons. Physics Reports, v. 317, n. 1, p. 1-142, 1999.

10 SHIFMAN, M.; VAINSHTEIN, A.; ZAKHAROV, V. QCD and resonance physics. Theoretical foundations. Nuclear Physics B, v. 147, n. 5, p. 385 - 447, 1979.

11 BAKER, G. A.; GRAVES-MORRIS, P. Padé approximants: encyclopedia of mathematics and it's applications. 2nd. ed. Cambridge: Cambridge University Press, 1996.

12 COPLEY, L. Mathematics for the physical sciences. Berlin: De Gruyter, 2015.

13 BAKER, G. A. et al. Essentials of Padé approximants. New York: Academic Press, 1975.

14 SAMUEL, M. A.; LI, G.; STEINFELDS, E. Estimating perturbative coefficients in quantum field theory using Padé approximants. Physics Letters B, v. 323, n. 2, p. 188 192, 1994.

15 SAMUEL, M. A.; LI, G. Estimating perturbative coefficients in high-energy physics and condensed matter theory. International Journal of Theoretical Physics, v. 33, n. 7, p. 1461-1469, 1994 . 
16 BAKER, G. A. et al. Ising-model critical indices in three dimensions from the Callan-Symanzik equation. Physical Review Letters, v. 36, n. 23, p. 1351-1354, 1976.

17 SAMUEL, M. A.; ELLIS, J.; KARLINER, M. Comparison of the Padé approximation method to perturbative QCD calculations. Physical Review Letters, v. 74, n. 22, p. 4380-4383, 1995.

18 BOITO, D.; MASJUAN, P.; OLIANI, F. Higher-order QCD corrections to hadronic $\tau$ decays from Padé approximants. Journal of High Energy Physics, v. 2018, n. 8, p. $075,2018$.

19 BENEKE, M.; BRAUN, V. M. Naive nonabelianization and resummation of fermion bubble chains. Physics Letters B, v. 348, n. 3, p. 513-520, 1995.

20 BROADHURST, D. J.; KATAEV, A. L.; MAXWELL, C. J. Renormalons and multiloop estimates in scalar correlators: Higgs decay and quark mass sum rules. Nuclear Physics B, v. 592, n. 1, p. 247-293, 2001.

21 BOITO, D.; JAMIN, M.; MIRAVITLLAS, R. Scheme variations of the QCD coupling and hadronic $\tau$ decays. Physical Review Letters, v. 117, n. 15, p. 152001, 2016.

22 FUKUDA, Y. et al. Evidence for oscillation of atmospheric neutrinos. Physical Review Letters, v. 81, n. 8, p. 1562-1567, 1998.

23 PICH, A. The Standard Model of electroweak interactions. In: EUROPEAN SCHOOL OF HIGH-ENERGY PHYSICS, 2006, Aronsborg. Proceedings [...]. Sweden: CERN, 2006. p. 1-49.

24 THOMSON, M. Modern particle physics. Cambridge: Cambridge University Press, 2013.

25 NAMBU, Y. Quasi-particles and gauge invariance in the theory of superconductivity. Physical Review, v. 117, n. 3, p. 648, 1960.

26 GOLDSTONE, J. Field theories with «Superconductor» solutions. Il Nuovo Cimento, v. 19, n. 1, p. 154-164, 1961.

27 GOldstone, J.; SAlAM, A.; WEInBERG, S. Broken symmetries. Physical Review, v. 127, n. 3, p. 965-970, 1962.

28 DJOUADI, A. The anatomy of electroweak symmetry breaking: tome I: the Higgs boson in the Standard Model. Physics Reports, v. 457, n. 1, p. 1 - 216, 2008.

29 PESKIN, M. E.; SCHROEDER, D. V. An introduction to quantum field theory. Reading, USA: Addison-Wesley, 1995.

30 JAMIN, M. QCD and renormalisation group methods. 2006. Available from: $<$ https://www.maria-laach.tp.nt.uni-siegen.de/downloads/files/2006/Jamin-2006.pdf $>$. Accessible at: 10 July 2019.

31 PASCUAL, P.; TARRACH, R. QCD: renormalization for the practitioner. Heidelberg: Springer-Verlag, 1984. 
32 'T HOOFT, G.; VELTMAN, M. Regularization and renormalization of gauge fields. Nuclear Physics B, v. 44, n. 1, p. 189 - 213, 1972.

33 BOLlini, C. G.; GIAMBIAGI, J. J. Dimensional renormalization: the number of dimensions as a regularizing parameter. Il Nuovo Cimento B, v. 12, n. 1, p. 20-26, 1972.

34 GREINER, W.; SCHRAMM, S.; STEIN, E. Quantum chromodynamics. 3rd ed. Heidelberg: Springer-Verlag, 2007.

35 BARDEEN, W. A. et al. Deep-inelastic scattering beyond the leading order in asymptotically free gauge theories. Physical Review D, v. 18, n. 11, p. 3998-4017, 1978.

36 BAIKOV, P.; CHETYRKIN, K.; KÜHN, J. Five-loop running of the QCD coupling constant. Physical Review Letters, v. 118, n. 8, p. 082002, 2017.

37 BAIKOV, P.; CHETYRKIN, K.; KÜHN, J. Quark mass and field anomalous dimensions to $\mathscr{O}\left(\alpha_{s}^{5}\right)$. Journal of High Energy Physics, v. 2014, n. 10, p. 076, 2014.

38 RITBERGEN, T. van; VERMASEREN, J.; LARIN, S. The four loop beta function in quantum chromodynamics. Physics Letters B, v. 400, n. 3, p. 379-384, 1997.

39 CHETYRKIN, K. Quark mass anomalous dimension to $\mathscr{O}\left(\alpha_{s}^{4}\right)$. Physics Letters B, v. 404, n. 1, p. 161-165, 1997.

40 VERMASEREN, J.; LARIN, S.; RITBERGEN, T. van. The four loop quark mass anomalous dimension and the invariant quark mass. Physics Letters B, v. 405, n. 3, p. 327-333, 1997.

41 JAMIN, M.; MIRAVITLLAS, R. Scalar correlator, Higgs decay into quarks, and scheme variations of the QCD coupling. Journal of High Energy Physics, v. 2016, n. 10, p. 059, 2016.

42 VERMASEREN, J.; LARIN, S.; van Ritbergen, T. The 4-loop quark mass anomalous dimension and the invariant quark mass. Physics Letters B, v. 405, n. 3, p. 327 - 333, 1997.

43 ZYLA, P. et al. Review of particle physics. Progress of Theoretical and Experimental Physics, v. 2020, n. 8, 2020. DOI: 10.1093/ptep/ptaa104.

44 CELMASTER, W.; GONSALVES, R. J. Renormalization-prescription dependence of the quantum-chromodynamic coupling constant. Physics Letters D, v. 20, n. 6, p. 1420-1434, 1979.

45 GROZIN, A. Lectures on QED and QCD: practical calculation and renormalization of one-and multi-loop Feynman diagrams. Singapore: World Scientific, 2007.

46 SMIRNOV, V. A. Evaluating Feynman integrals. Berlin: Springer Science \& Business Media, 2004. (Springer tracts in modern physics, v. 211).

47 BRAATEN, E.; LEVEILlE, J. P. Higgs-boson decay and the running mass. Physical Review D, v. 22, n. 3, p. 715-721, 1980. 
48 SAKAI, N. Perturbative quantum-chromodynamic corrections to the hadronic decay width of the Higgs boson. Physical Review D, v. 22, n. 9, p. 2220-2223, 1980.

49 GORISHNY, S. G. et al. Three-loop QCD correction to the correlator of the quark scalar currents and $\Gamma_{t o t}\left(H^{0} \rightarrow\right.$ hadrons). Modern Physics Letters A, v. 5, n. 32, p. 2703-2711, 1990.

50 DYSON, F. Divergence of perturbation theory in quantum electrodynamics. Physical Review, v. 85, n. 4, p. 631-632, 1952.

51 LIPATOV, L. Divergence of the perturbation theory series and the quasiclassical theory. Soviet Physics - journal of experimental and theoretical physics, v. 45, n. 2, p. 216-223, 1977.

52 SHIFMAN, M. New and old about renormalons: in memoriam Kolya Uraltsev. International Journal of Modern Physics A, v. 30, n. 10, p. 1543001, 2015.

53 NEUBERT, M. Scale setting in QCD and the momentum flow in Feynman diagrams. Physical Review D, v. 51, n. 10, p. 5924-5941, 1995.

54 MARINO, M. Instantons and large N: an introduction to non-perturbative methods in quantum field theory. Cambridge: Cambridge University Press, 2015.

55 BENEKE, M.; JAMIN, M. $\alpha_{s}$ and the $\tau$ hadronic width: fixed-order, contourimproved and higher-order perturbation theory. Journal of High Energy Physics, v. 2008, n. 9, p. 044-044, 2008.

56 MASJUAN, P.; PERIS, S. A rational approach to resonance saturation in large- $N_{c}$ QCD. Journal of High Energy Physics, v. 2007, n. 5, p. 040, 2007.

57 BENEKE, M. Large-order perturbation theory for a physical quantity. Nuclear Physics B, v. 405, n. 2, p. $424-450,1993$.

58 GROZIN, A. G. Renormalons: technical introduction. 2003. Available from: $<$ https://arxiv.org/pdf/hep-ph/0311050.pdf>. Accessible at: 03 Dec. 2020.

59 KATAEV, A. Light quark masses and renormalons. In: INTERNATIONAL SEMINAR ON HIGH-ENERGY PHYSICS, 11, 2000, Pushkin. Proceedings [...]. Sweden: CERN, 2002. p. 35-52.

60 CHETYRKIN, K.; MAIER, A. Massless correlators of vector, scalar and tensor currents in position space at orders $\alpha_{s}^{3}$ and $\alpha_{s}^{4}$ : explicit analytical results. Nuclear Physics B, v. 844, n. 2, p. 266 - 288, 2011.

61 HERZOG, F. et al. The five-loop beta function of Yang-Mills theory with fermions. Journal of High Energy Physics, v. 2017, n. 2, p. 90, 2017.

62 BAKULEV, A.; MIKHAILOV, S. Resummation in (F)APT. 2008. Available from: $<$ https://arxiv.org/pdf/0803.3013.pdf>. Accessible at: 05 Jan. 2021.

63 BAKUlEV, A. P.; MIKHAILOV, S. V.; STEFANIS, N. G. Higher-order QCD perturbation theory in different schemes: from FOPT to CIPT to FAPT. Journal of High Energy Physics, v. 2010, n. 6, p. 85, 2010. 
64 KATAEV, A. L.; STARSHENKO, V. V. Estimates of the higher order QCD corrections to $R(s), R_{\tau}$ and deep inelastic scattering sum rules. Modern Physics Letters A, v. 10, n. 3, p. 235-250, 1995.

65 DU, B.-L. et al. Extending the predictive power of perturbative QCD. The European Physical Journal C, v. 79, n. 3, p. 182, 2019.

66 WU, X.-G. et al. The QCD renormalization group equation and the elimination of fixed-order scheme-and-scale ambiguities using the principle of maximum conformality. Progress in Particle and Nuclear Physics, v. 108, p. 103706, 2019. DOI: 10.1016/j.ppnp.2019.05.003.

67 CHETYRKIN, K.; KÜHN, J.; STEINHAUSER, M. RunDec: a Mathematica package for running and decoupling of the strong coupling and quark masses. Computer Physics Communications, v. 133, n. 1, p. 43 - 65, 2000.

68 MONDINI, R.; SCHIAVI, M.; WILLIAMS, C. $\mathrm{N}^{3}$ LO predictions for the decay of the Higgs boson to bottom quarks. Journal of High Energy Physics, v. 2019, n. 6, p. 79, 2019. 